\title{
Interval Type-2 Fuzzy Brain Emotional Control Design for the Synchronization of 4D Nonlinear Hyperchaotic Systems
}

Tuan-Tu Huynh

Yuan Ze University

Chih-Min Lin ( $\nabla$ cml@saturn.yzu.edu.tw )

Yuan Ze University

Tien-Loc Le

Lac Hong University

Mai The Vu

Sejong University

Fei Chao

Xiamen University

\section{Research Article}

Keywords: Interval type-2 fuzzy system, fourfold function-link network, fuzzy brain emotional controller, 4D nonlinear hyperchaotic system

Posted Date: June 7th, 2021

DOI: https://doi.org/10.21203/rs.3.rs-585893/v1

License: (c) (1) This work is licensed under a Creative Commons Attribution 4.0 International License. Read Full License 


\title{
Interval Type-2 Fuzzy Brain Emotional Control Design for the Synchronization of 4D Nonlinear Hyperchaotic Systems
}

\author{
Tuan-Tu Huynh a, b, $*$, Chih-Min Lin ${ }^{\text {a }} *$, Tien-Loc Le ${ }^{\text {b }}$, Mai The Vu ${ }^{\text {c }}$, Fei Chao ${ }^{d}$ \\ a Department of Electrical Engineering, Yuan Ze University, No. 135, Yuandong Road, Zhongli 320, Taoyuan, Taiwan, ROC. \\ b Department of Electrical Electronic and Mechanical Engineering, Lac Hong University, No. 10, Huynh Van Nghe Road, Bien Hoa, \\ Vietnam. \\ c School of Intelligent Mechatronics Engineering, Sejong University, 98 Gunja-dong, Gwangjin-gu, Seoul, 143-747, South Korea. \\ d Department of Cognitive Science, Xiamen University, China. \\ * Corresponding author Chih-Min Lin (cml@saturn.yzu.edu.tw) and Tuan-Tu Huynh (huynhtuantu@saturn.yzu.edu.tw).
}

Abstract - This research provides a novel intelligent control structure for 4D nonlinear hyperchaotic systems. This is a hybrid design containing a new interval type-2 fuzzy fourfold function-link brain emotional controller and a smooth robust controller. It comprises a fuzzy inference system and three subnetworks. The subnetworks are a new fourfold function-link network, a type-2 fuzzy amygdala network and a type-2 fuzzy prefrontal cortex network that decrease the synchronization errors efficiently, follow the reference signal well and achieve good performance. Two Lyapunov stability functions are utilized to get the adaptive laws, and they are applied to online tune the parameters of the system. The proposed design is used to synchronize two 4D nonlinear hyperchaotic systems and the simulation results are given to demonstrate its superiority and effectiveness.

Keywords - Interval type-2 fuzzy system, fourfold function-link network, fuzzy brain emotional controller, 4D nonlinear hyperchaotic system

\section{Introduction}

Over the years, chaos studies had a strong influence on the development of global science and technology, in which chaotic synchronization is one of the interesting topics that attract many scholars (Hsu et al. 2009; Sothmann et al. 2012; Sun et al. 2013; Vaidyanathan and Rasappan 2014; Wang et al. 2019a; Wu et al. 2017). Synchronization of chaotic systems is described as the phenom that happens when a master system controls a slave system by tuning a given characteristic of their motion (Lin and Huynh; Wang et al. 2019b). Nowadays, various chaotic and hyperchaotic systems have been investigated in many fields (Chen et al. 2018; Panahi et al. 2019; Pham et al. 2017). Particularly, the 4D hyperchaotic systems comprising complex shapes of equilibrium points are studied in recent years (Rakheja et al. 
2019; Sambas et al. 2018; Vaidyanathan et al. 2018). The first Lyapunov exponent is generally used to define the disorders of chaotic systems. Moreover, the studies of chaos with hidden attractions are significant since they can create undesirable and harmful problems with minor changes in dynamics such as engine system (Marzbanrad and Babalooei 2016), airplane system (Andrievsky et al. 2018), transportation system (Adeli and Jiang 2008), electro-mechanical system (Xue et al. 2019), radar system (Beal et al. 2016), and bridge system (Ni et al. 2019). Recently, many control systems have been proposed for nonlinear chaotic systems to achieve good control performance such as an adaptive fuzzy control (Sambas et al. 2020), a passive control (Sambas et al. 2019a), an active backstepping control (Sambas et al. 2021), an adaptive control (Sambas et al. 2019b), an integral sliding mode control (Vaidyanathan et al. 2019), a double function-link brain emotional control (Huynh et al. 2020c), a modified grey wolfbased multilayer type-2 asymmetric fuzzy control (Le et al. 2020b), a self-organizing interval type-2 fuzzy asymmetric cerebellar model articulation control (Le et al. 2020a), a wavelet interval type-2 fuzzy brain emotional control (Huynh et al. 2020a), and a brain-imitated neural network control (Lin et al. 2021).

A function-link network (FLN) is a kind of feed-forward network model that is efficiently applied for function approximation with quick convergence speed and less computational load (Patra and Pal 1995). In the past year, many scholars have used the FLN in their researches to achieve better results (Huynh et al. 2019; Lin and Huynh; Zhou et al. 2018). A single FLN was used to adjust the weights for two independent networks in a brain emotional learning network (BELN) that are the amygdala and orbitofrontal cortex networks. However, it is necessary to attain the BELN's weights precisely and their values separately. Recently, a dual FLN was proposed to improve this drawback (Huynh and Lin 2019; Lin et al. 2021). In this research, a new fourfold FLN is designed for the proposed type-2 fuzzy BELN's structure.

LeDoux proposed a brain emotional learning network (BELN), which is a computational simulation system describing the data processing scheme of the mammal brain (LeDoux 1991). A BELN connects a stimulus to the equivalent emotional reaction appearing in an amygdala of a brain. The brain has an amygdala and an orbitofrontal cortex so that an output of the BELN is associated among the two networks, which influence each other. For that reason, BELN still works well with system uncertainty with fast learning speeds and good approximation capabilities, and it can reduce tracking errors effectively. Over the years, some remarkable studies have applied BELN in different fields (Dashti et al. 2017; Hsu et al. 2016; Kong et al. 2019; Le et al. 2018).

So far, intelligent controllers based on type 1 (T1) and type 2 (T2) platforms have been developed for different applications in different fields (Boubellouta et al. 2019; Lin and Huynh 2019; Mendel et al. 
2019; Zhao and Lin 2019). Since T1-fuzzy logic systems (FLSs) is required to have membership functions that are well defined, it cannot thoroughly handle the large uncertainty of inputs and parameters of nonlinear systems well. To overcome this drawback, T2-FLS and interval T2-FLS are typically used as they have general expanded features than T1-FLS, more freely generated for better control performance and improved response to uncertain input of membership functions (Le 2019; Rong et al. 2018). In order to design effective networks, many researches have come up with solutions that combine different efficient techniques (Lin et al. 2018; Wang et al. 2017), additional functional networks (Ding et al. 2019; Huynh et al. 2020b; Huynh et al. 2019) and extra algorithms (Huynh et al. 2018; Rahmani et al. 2018; Ravi et al. 2017) to build networks automatically.

This study develops a new more efficient interval type-2 fuzzy fourfold function-link brain emotional controller (IT2FFFLBC) for 4D nonlinear hyperchaotic systems. The proposed IT2FFFLBC control system includes a IT2FFFLBC and a smooth robust controller. The IT2FFFLBC is used as the main controller and a smooth robust controller is used to eliminate the approximate error term and to warrant the system stability. The main contributions of this research are summarized as follows: 1) The proposed IT2FFFLBC comprises a set of fuzzy inference rules and three subnetworks that are the fourfold function-link network, the type-2 fuzzy prefrontal cortex network, and the type-2 fuzzy amygdala network to efficiently reduce the synchronization error and achieve good performance. (2) A new fourfold FLN is designed to tune the particular weights for the type-2 fuzzy structures of the orbitofrontal cortex and amygdala of the proposed IT2FFFLBC. (3) Effective adaptive learning laws for updating the system parameters effectively are obtained from two Lyapunov functions, and they are also used to prove the stability of the system. (4) Finally, the simulation results and some comparisons in root mean square error with former studies for a 4D hyperchaotic Lorenz-Lu system and a 4D hyperchaotic Rikitake two-wing dynamo system have shown the effectiveness and advantage of the proposed control system.

\section{Theoretical Problems}

The master system (MS) for a $n$th order 4D nonlinear hyperchaotic system is defined as:

$\boldsymbol{x}_{M S}^{(n)}(t)=\boldsymbol{f}_{M S}\left(\overline{\boldsymbol{x}}_{M S}(t)\right)$

The slave system (SS) is given as:

$\boldsymbol{y}_{S S}^{(n)}(t)=\boldsymbol{f}_{S S}\left(\overline{\boldsymbol{y}}_{S S}(t)+\boldsymbol{u}_{S S}(t)+\boldsymbol{n}_{S S}(t)\right)$

where

\begin{tabular}{|c|c|c|c|c|c|c|}
\hline $\boldsymbol{x}_{M S}(t) @\left[x_{M S 1}(t), x_{M S 2}(t), \mathrm{K}, x_{M S m}(t)\right.$ & $\in \mathfrak{R}^{m}$ & is & the & output & for & the \\
\hline $\boldsymbol{y}_{S S}(t) @\left[y_{S S 1}(t), y_{S S 2}(t), \mathrm{K}, y_{S S m}(t)\right]$ & $\in \mathfrak{R}^{m}$ & is & the & output & for & the \\
\hline$\overline{\boldsymbol{x}}_{M S}(t) @\left[\boldsymbol{x}_{M S}^{T}(t), \boldsymbol{x}_{M S}^{T}(t), \mathrm{K}, \boldsymbol{x}_{M S}^{(n-1) T}(t)\right]^{T}$ & $\in \mathfrak{R}^{m n}$ & is & the & state & for & the \\
\hline
\end{tabular}


$\overline{\boldsymbol{y}}_{S S}(t) @\left[\boldsymbol{y}_{S S}^{T}(t), \boldsymbol{y}_{S S}(t), \mathrm{K}, \boldsymbol{y}_{S S}^{(n-1) T}(t)\right]^{T} \in \mathfrak{R}^{m n} \quad$ is $\quad$ the $\quad$ state $\quad$ for $\quad$ the $\mathrm{SS}$. $\boldsymbol{f}_{M S}\left(\overline{\boldsymbol{x}}_{M S}(t)\right) \in \mathfrak{R}^{m}, \boldsymbol{f}_{S S}\left(\overline{\boldsymbol{y}}_{S S}(t)\right) \in \mathfrak{R}^{m}$ are the unknown bounded nonlinear functions. $\boldsymbol{u}_{S S}(t) @\left[u_{S S 1}(t), u_{S S 2}(t), \mathrm{K}, u_{S S m}(t)\right]^{T} \in \mathfrak{R}^{m}$ is the control input for the SS. $\boldsymbol{n}_{S S}(t)=\left[n_{S S 1}(t), n_{S S 2}(t), \mathrm{K}, n_{S S m}(t)\right]^{T} \in \mathfrak{R}^{m}$ is the unknown bounded external noise for the SS, where $m$ is the number of outputs and inputs of the system, and the subscript letters $S S$ and $M S$ indicate the slave system and master system, respectively.

The synchronization error state is defined as follows:

$\boldsymbol{e}_{S y n c}(t) @ \boldsymbol{y}_{S S}(t)-\boldsymbol{x}_{M S}(t) @\left[e_{1}(t), e_{2}(t), \mathrm{K}, e_{m}(t)\right]^{T} \in \mathfrak{R}^{m}$

The error vector is then defined as:

$\overline{\boldsymbol{e}}_{S S}(t) @\left[\mathbb{S}_{S y n c}, \mathrm{~K}, \boldsymbol{e}_{S y n c}^{(n-1) T}\right]^{T} \in \mathfrak{R}^{m n}$

If the unknown bounded nonlinear functions $\boldsymbol{f}_{M S}\left(\overline{\boldsymbol{x}}_{M S}(t)\right), \boldsymbol{f}_{S S}\left(\overline{\boldsymbol{y}}_{S S}(t)\right)$, and the external noise $\boldsymbol{n}_{S S}(t)$ of the SS are known, the ideal controller is then determined as:

$\boldsymbol{u}_{I D C}^{*}(t)=-\boldsymbol{f}_{S S}\left(\overline{\boldsymbol{y}}_{S S}(t)+\boldsymbol{y}_{S S}^{(n)}(t)-\boldsymbol{n}_{S S}(t)+\overline{\boldsymbol{K}}^{T} \overline{\boldsymbol{e}}_{S S}\right.$

where $\overline{\boldsymbol{K}} @\left[\boldsymbol{K}_{1}, \boldsymbol{K}_{2}, \mathrm{~K}, \boldsymbol{K}_{n}\right]^{T} \in \mathfrak{R}^{m n \times m}$ is the gain matrix with real values.

Inserting (5) into (2), attain the following error dynamic condition

$\boldsymbol{e}_{\text {Sync }}^{(n)}+\overline{\boldsymbol{K}}^{T} \overline{\boldsymbol{e}}_{S S}=0$

If $\overline{\boldsymbol{K}}$ is suitably determined to satisfy the Hurwitz stability criterion to produce the roots on the left side of the complex plane, i.e. $\lim _{t \rightarrow \infty} \overline{\boldsymbol{e}}_{S S}(t) \rightarrow 0$. Due to $\boldsymbol{f}_{M S}\left(\overline{\boldsymbol{x}}_{M S}(t)\right), \boldsymbol{f}_{S S}\left(\overline{\boldsymbol{y}}_{S S}(t)\right)$ and $\boldsymbol{n}_{S S}(t)$ are unknown, $\boldsymbol{u}_{I D C}^{*}(t)$ in (5) is unattainable. As a result, an IT2FFFLBC is used to imitate $\boldsymbol{u}_{I D C}^{*}(t)$.

3. Interval Type-2 Fuzzy Fourfold Function-Link Brain Emotional Controller 1)Fourfold Function-Link Network

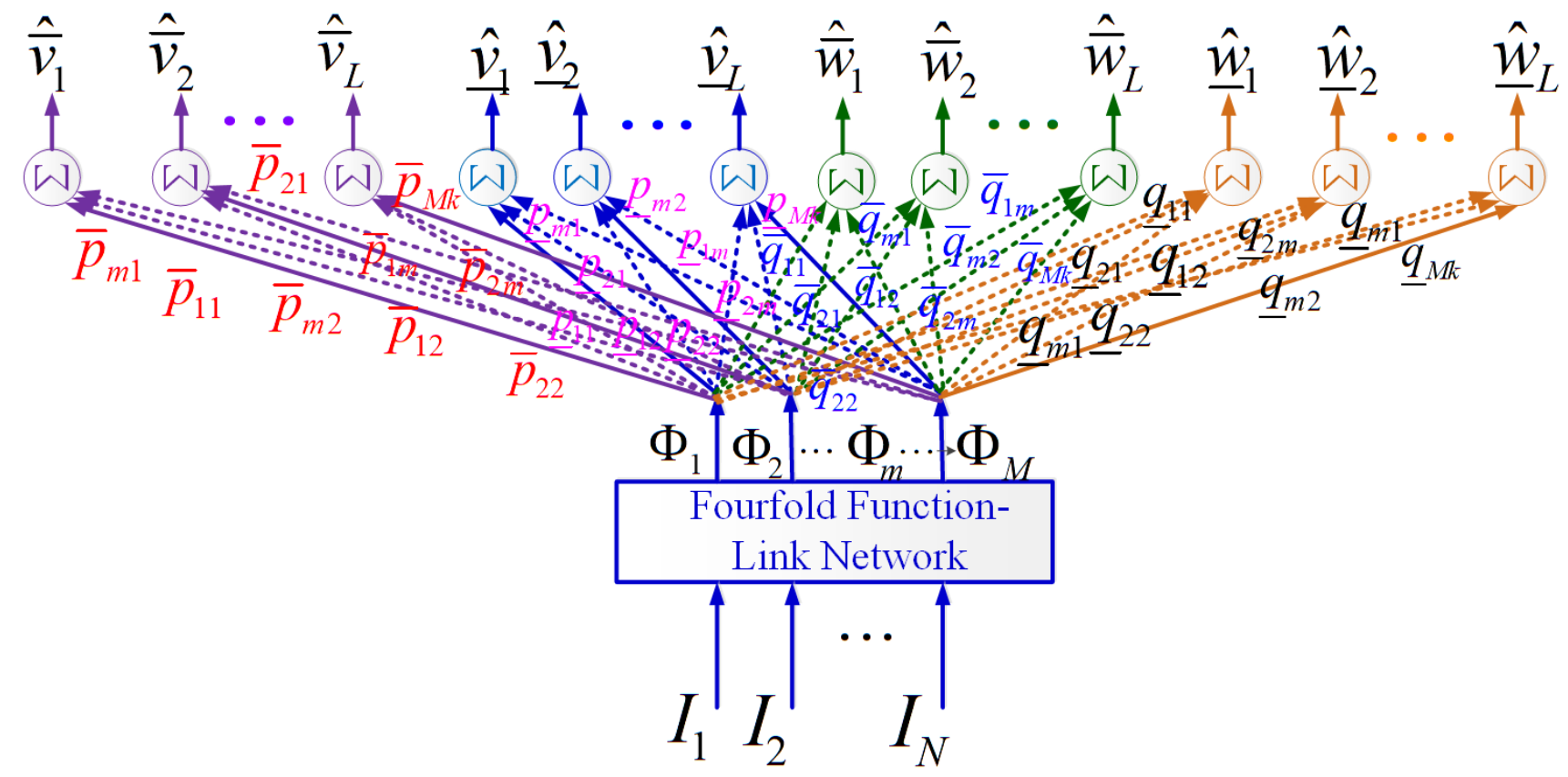

Fig. 1. Fourfold FLN 
This study proposes a new fourfold FLN (FFLN) to expand the processing ability of the IT2FFFLBC. The main FFLN duty is to enhance accuracy for lower and upper weights of the type-2 fuzzy orbitofrontal cortex and amygdala networks of the proposed structure. The FFLN structure is displayed in Fig. 1, where the FFLN operates on input variables by producing a linearly independent set. The proposed FFLN uses the cosine and sine functions because of their simple and clear functions and rapidly computed. Define $\boldsymbol{I} @\left[\begin{array}{llll}I_{1} & I_{2} & \mathrm{~K} & I_{N}\end{array}\right]^{T} \in \mathfrak{R}^{N}$, the inputs are then allocated in the extended space as follows: $\boldsymbol{\Phi}=\left[\begin{array}{llll}\Phi_{1} & \Phi_{2} & \mathrm{~K} & \Phi_{M}\end{array}\right]^{T} \in \mathfrak{R}^{M}$, where $M$ and $N$ are respectively the number of the outputs and the total number of input signals. Particularly, if the input is $I=\left[I_{1}, I_{2}\right]^{T}$, then $\boldsymbol{\Phi}=\left(1, I_{1}, \cos \left(\pi \times I_{1}\right), \sin \left(\pi \times I_{1}\right), I_{2}, \quad \cos \left(\pi \times I_{2}\right), \sin \left(\pi \times I_{2}\right), I_{1} \times I_{2}\right)$. Next, the FFLN outputs are determined as:

$$
\begin{aligned}
& \hat{\boldsymbol{w}}_{k}=\underline{q}_{1 k} \Phi_{1}+\mathrm{K}+\underline{q}_{m k} \Phi_{m}+\mathrm{K}+\underline{q}_{M k} \Phi_{M}=\sum_{m=1}^{M} \underline{q}_{m k} \Phi_{m}=\underline{\boldsymbol{q}}_{k}^{T} \boldsymbol{\Phi} \\
& \hat{\overline{\boldsymbol{w}}}_{k}=\bar{q}_{1 k} \Phi_{1}+\mathrm{K}+\bar{q}_{m k} \Phi_{m}+\mathrm{K}+\bar{q}_{M k} \Phi_{M}=\sum_{m=1}^{M} \bar{q}_{m k} \Phi_{m}=\overline{\boldsymbol{q}}_{k}^{T} \boldsymbol{\Phi} \\
& \underline{\hat{\boldsymbol{v}}}_{k}=\underline{p}_{1 k} \Phi_{1}+\mathrm{K}+\underline{p}_{m k} \Phi_{m}+\mathrm{K}+\underline{p}_{M k} \Phi_{M}=\sum_{m=1}^{M} \underline{p}_{m k} \Phi_{m}=\underline{\boldsymbol{p}}_{k}^{T} \boldsymbol{\Phi} \\
& \hat{\hat{\boldsymbol{v}}_{k}}=\bar{p}_{1 k} \Phi_{1}+\mathrm{K}+\bar{p}_{m k} \Phi_{m}+\mathrm{K}+\bar{p}_{M k} \Phi_{M}=\sum_{m=1}^{M} \bar{p}_{m k} \Phi_{m}=\overline{\boldsymbol{p}}_{k}^{T} \boldsymbol{\Phi} \\
& \text { for } m=1,2, \mathrm{~K}, M, k=1,2, \mathrm{~K}, L,
\end{aligned}
$$

where $\Phi_{m}$ is the $m$-th function expansion output, $\hat{\overline{\boldsymbol{v}}}_{k}, \hat{\overline{\boldsymbol{w}}}_{k}, \hat{\boldsymbol{v}}_{k}$, and $\underline{\hat{\boldsymbol{w}}}_{k}$ are the output of FFLN, $\bar{p}_{m k}, \bar{q}_{m k}, \underline{p}_{m k}$, and $\underline{q}_{m k}$ are the connective weight among $\hat{\bar{v}}_{k}, \hat{\overline{\boldsymbol{w}}}_{k}, \underline{\hat{v}}_{k}, \underline{\hat{w}}_{k}$ and $\Phi_{m}$, and connective weight vectors are defined as

$$
\begin{aligned}
& \overline{\boldsymbol{p}}_{k}=\left[\bar{p}_{1 k}, \mathrm{~K}, \bar{p}_{m k}, \mathrm{~K}, \bar{p}_{M k}\right] \in \mathfrak{R}^{M}, \\
& \underline{\boldsymbol{p}}_{k}=\left[\underline{p}_{1 k}, \mathrm{~K}, \underline{p}_{m k}, \mathrm{~K}, \underline{p}_{M k}\right] \in \mathfrak{R}^{M}, \\
& \overline{\boldsymbol{q}}_{k}=\left[\bar{q}_{1 k}, \ldots, \bar{q}_{m k}, \ldots, \bar{q}_{M k}\right] \in \mathfrak{R}^{M} \\
& \underline{\boldsymbol{q}}_{k}=\left[\underline{q}_{1 k}, \ldots, \underline{q}_{m k}, \ldots, \underline{q}_{M k}\right] \in \mathfrak{R}^{M}
\end{aligned}
$$




\section{2) Interval Type-2 Fuzzy Fourfold Function-Link Brain Emotional Controller}

\section{Layer 1 Layer $2 \quad$ Layer $3 \quad$ Layer $4 \quad$ Layer 5}
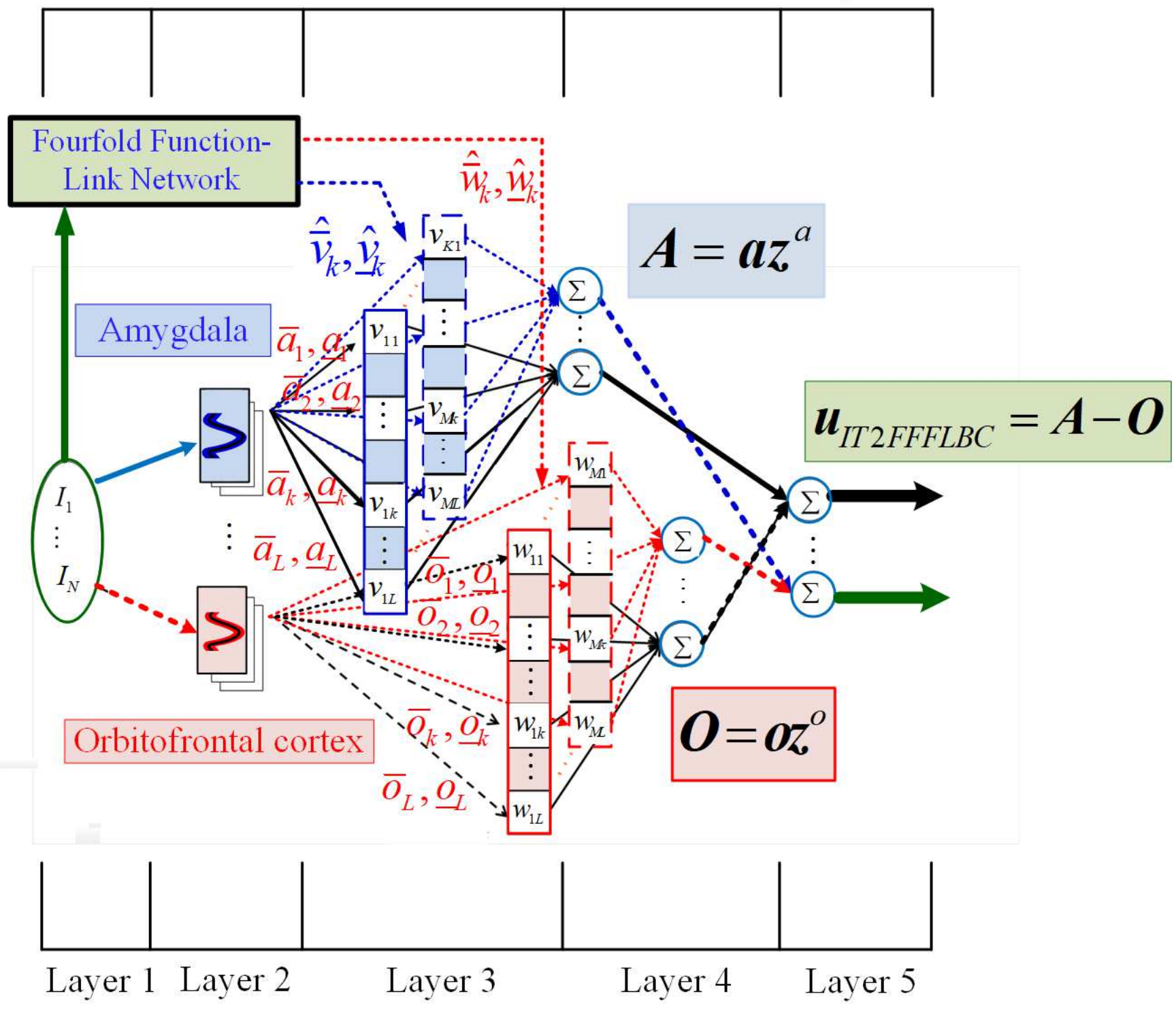

Fig. 2. Structure of IT2FFFLBC

The structure of IT2FFFLBC is shown in Fig. 2, which is composed of five layers: input layer, fuzzy membership function layer, output weight layer using FFLN, amygdala- orbitofrontal layer and output layer. The signal propagation in each layer is described below:

\section{Layer 1: Input layer}

$$
\boldsymbol{I}=\left[I_{1}, \mathrm{~L}, I_{i}, \mathrm{~L} I_{N}\right]^{T} \in \mathfrak{R}^{N} \text {, for } i=1,2, \ldots, N
$$

where $I_{i}$ is the $i$-th input.

\section{Layer 2: Fuzzy membership function layer}




$$
\begin{aligned}
& \bar{a}_{i k}=\bar{o}_{i k}=\exp \left[\frac{-\left(I_{i}-m_{i k}\right)^{2}}{2 \bar{\sigma}_{i k}^{2}}\right], \text { for } k=1,2, \ldots, L \\
& \underline{a}_{i k}=\underline{o}_{i k}=\exp \left[\frac{-\left(I_{i}-m_{i k}\right)^{2}}{2 \underline{\sigma}_{i k}^{2}}\right], \text { for } k=1,2, \ldots, L
\end{aligned}
$$

where $\bar{a}_{i k}, \bar{o}_{i k}$ and $\underline{a}_{i k}, \underline{o}_{i k}$ are the upper and lower values for the type-2 Gaussian membership function. $\sigma_{i k}$ is the variance, $m_{i k}$ is the mean, and $L$ is the number of layers.

$$
\begin{aligned}
& \bar{a}_{k}=\prod_{i=1}^{N} \bar{a}_{i k} \\
& \bar{o}_{k}=\prod_{i=1}^{N} \bar{o}_{i k} \\
& \underline{a}_{k}=\prod_{i=1}^{N} \underline{a}_{i k} \\
& \underline{o}_{k}=\prod_{i=1}^{N} \underline{o}_{i k}
\end{aligned}
$$

The proposed structure utilizes the following fuzzy inference rules:

$R^{k}:\left\{\begin{array}{l}\text { If } I_{1} \text { is } a_{1 k}, I_{2} \text { is } a_{2 k}, \mathrm{~L}, \text { and } I_{N} \text { is } a_{N k} \text {, then } \hat{v}_{k}=\boldsymbol{p}_{k}^{T} \boldsymbol{\Phi}, \text { for } i=1,2, \mathrm{~L}, N \\ \text { If } I_{1} \text { is } o_{1 k}, I_{2} \text { is } o_{2 k}, \mathrm{~L}, \text { and } I_{N} \text { is } o_{N k}, \text { then } \hat{w}_{k}=\boldsymbol{q}_{k}^{T} \boldsymbol{\Phi} \text {, for } k=1,2, \mathrm{~L}, L\end{array}\right.$

\section{Layer 3: Output weight layer using FFLN}

$$
\begin{aligned}
& \underline{\mathbf{w}}=\left[\hat{\underline{w}}_{1}, \mathrm{~K}, \hat{\underline{w}}_{k}, \mathrm{~K}, \hat{\underline{w}}_{L}\right]^{T} \in \mathfrak{R}^{L} \\
& \overline{\mathbf{w}}=\left[\hat{\bar{w}}_{1}, \mathrm{~K}, \hat{\bar{w}}_{k}, \mathrm{~K}, \hat{\bar{w}}_{L}\right]^{T} \in \mathfrak{R}^{L} \\
& \underline{\boldsymbol{v}}=\left[\hat{\hat{v}}_{1}, \mathrm{~K}, \hat{\hat{v}}_{k}, \mathrm{~K}, \hat{\hat{v}}_{L}\right]^{T} \in \mathfrak{R}^{L} \\
& \overline{\boldsymbol{v}}=\left[\hat{\bar{v}}_{1}, \mathrm{~K}, \hat{\bar{v}}_{k}, \mathrm{~K}, \hat{\bar{v}}_{L}\right]^{T} \in \mathfrak{R}^{L}
\end{aligned}
$$

where $\underline{\boldsymbol{w}}, \overline{\boldsymbol{w}}$ and $\underline{\boldsymbol{v}}, \overline{\boldsymbol{v}}$ are respectively the weights of orbitofrontal and amygdala; and they are defined as:

$$
\underline{\boldsymbol{w}}=\left[\begin{array}{c}
\hat{w}_{1} \\
\mathrm{M} \\
\hat{w}_{k} \\
\mathrm{M} \\
\hat{\hat{w}}_{L}
\end{array}\right]=\left[\begin{array}{ccccc}
\underline{q}_{11} & \mathrm{~L} & \underline{q}_{m 1} & \mathrm{~L} & \underline{q}_{M 1} \\
\mathrm{M} & \mathrm{O} & \mathrm{M} & \mathrm{O} & \mathrm{M} \\
\underline{q}_{1 k} & \mathrm{~L} & \underline{q}_{m k} & \mathrm{~L} & \underline{q}_{M k} \\
\mathrm{M} & \mathrm{O} & \mathrm{M} & \mathrm{O} & \mathrm{M} \\
\underline{q}_{1 L} \mathrm{~L} & \underline{q}_{m L} & \mathrm{~L} & \underline{q}_{M L}
\end{array}\right]\left[\begin{array}{c}
\Phi_{1} \\
\mathrm{M} \\
\Phi_{m} \\
\mathrm{M} \\
\Phi_{M}
\end{array}\right]=\underline{\boldsymbol{q}}^{T} \boldsymbol{\Phi}
$$




$$
\begin{aligned}
& \overline{\boldsymbol{w}}=\left[\begin{array}{c}
\hat{\bar{w}}_{1} \\
\mathrm{M} \\
\hat{\bar{w}}_{k} \\
\mathrm{M} \\
\hat{\bar{w}}_{L}
\end{array}\right]=\left[\begin{array}{ccccc}
\bar{q}_{11} & \mathrm{~L} & \bar{q}_{m 1} & \mathrm{~L} & \bar{q}_{M 1} \\
\mathrm{M} & \mathrm{O} & \mathrm{M} & \mathrm{O} & \mathrm{M} \\
\bar{q}_{1 k} & \mathrm{~L} & \bar{q}_{m k} & \mathrm{~L} & \bar{q}_{M k} \\
\mathrm{M} & \mathrm{O} & \mathrm{M} & \mathrm{O} & \mathrm{M} \\
\bar{q}_{1 L} \mathrm{~L} & \bar{q}_{m L} & \mathrm{~L} & \bar{q}_{M L}
\end{array}\right]\left[\begin{array}{c}
\Phi_{1} \\
\mathrm{M} \\
\Phi_{m} \\
\mathrm{M} \\
\Phi_{M}
\end{array}\right]=\overline{\boldsymbol{q}}^{T} \boldsymbol{\Phi} \\
& \underline{\boldsymbol{v}}=\left[\begin{array}{c}
\hat{v}_{1} \\
\mathrm{M} \\
\hat{\underline{v}}_{k} \\
\mathrm{M} \\
\hat{\underline{v}}_{L}
\end{array}\right]=\left[\begin{array}{ccccc}
\underline{p}_{11} & \mathrm{~L} & \underline{p}_{m 1} & \mathrm{~L} & \underline{p}_{M 1} \\
\mathrm{M} & \mathrm{O} & \mathrm{M} & \mathrm{O} & \mathrm{M} \\
\underline{p}_{1 k} \mathrm{~L} & \underline{p}_{m k} & \mathrm{~L} & \underline{p}_{M k} \\
\mathrm{M} & \mathrm{O} & \mathrm{M} & \mathrm{O} & \mathrm{M} \\
\underline{p}_{1 L} \mathrm{~L} & \underline{p}_{m L} & \mathrm{~L} & \underline{p}_{M L}
\end{array}\right]\left[\begin{array}{c}
\Phi_{1} \\
\mathrm{M} \\
\Phi_{m} \\
\mathrm{M} \\
\Phi_{M}
\end{array}\right]=\underline{\boldsymbol{p}}^{T} \boldsymbol{\Phi} \\
& \overline{\boldsymbol{v}}=\left[\begin{array}{c}
\hat{\bar{v}}_{1} \\
\mathrm{M} \\
\hat{\bar{v}}_{k} \\
\mathrm{M} \\
\hat{\bar{v}}_{L}
\end{array}\right]=\left[\begin{array}{ccccc}
\bar{p}_{11} & \mathrm{~L} & \bar{p}_{m 1} & \mathrm{~L} & \bar{p}_{M 1} \\
\mathrm{M} & \mathrm{O} & \mathrm{M} & \mathrm{O} & \mathrm{M} \\
\bar{p}_{1 k} \mathrm{~L} & \bar{p}_{m k} & \mathrm{~L} & \bar{p}_{M k} \\
\mathrm{M} & \mathrm{O} & \mathrm{M} & \mathrm{O} & \mathrm{M} \\
\bar{p}_{1 L} \mathrm{~L} & \bar{p}_{m L} & \mathrm{~L} & \bar{p}_{M L}
\end{array}\right]\left[\begin{array}{c}
\Phi_{1} \\
\mathrm{M} \\
\Phi_{m} \\
\mathrm{M} \\
\Phi_{M}
\end{array}\right]=\overline{\boldsymbol{p}}^{T} \boldsymbol{\Phi}
\end{aligned}
$$

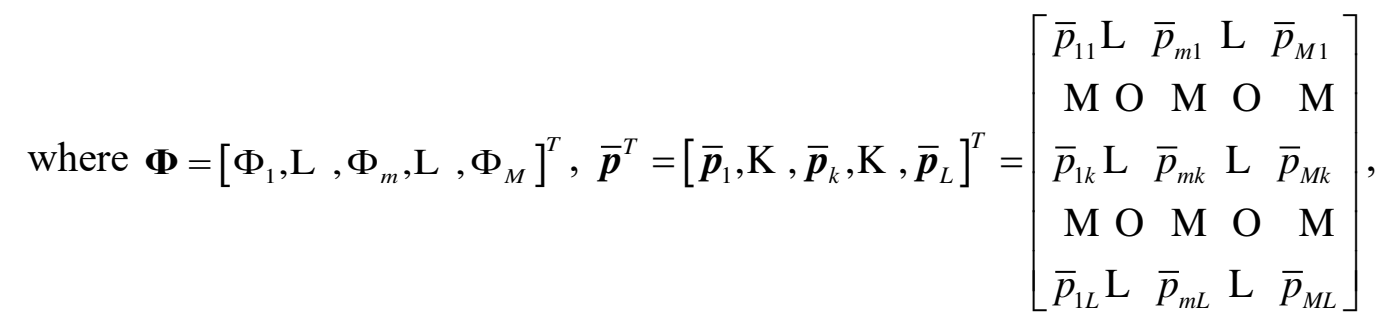

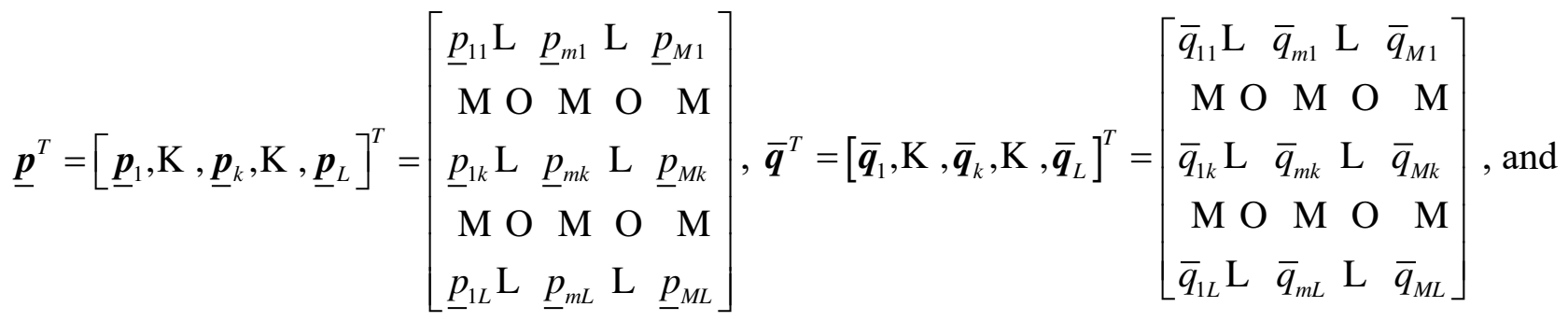

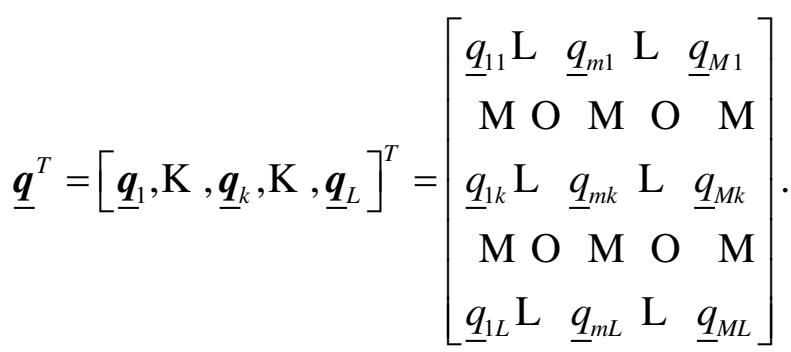

Layer 4: Amygdala- orbitofrontal layer 


$$
\begin{array}{r}
a_{k}^{l}=\frac{\sum_{k=1}^{L} a_{k}^{l} \bar{v}_{k}}{\sum_{k=1}^{L} a_{k}^{l}}=\frac{\sum_{k=1}^{L} a_{k}^{l} \overline{\boldsymbol{p}}_{k}^{T} \mathbf{\Phi}}{\sum_{k=1}^{L} a_{k}^{l}} \\
a_{k}^{r}=\frac{\sum_{k=1}^{L} a_{k}^{r} \underline{v}_{k}}{\sum_{k=1}^{L} a_{k}^{r}}=\frac{\sum_{k=1}^{L} a_{k}^{r} \underline{\boldsymbol{p}}_{k}^{T} \boldsymbol{\Phi}}{\sum_{k=1}^{L} a_{k}^{r}} \\
o_{k}^{l}=\frac{\sum_{k=1}^{L} o_{k}^{l} \bar{v}_{k}}{\sum_{k=1}^{L} o_{k}^{l}}=\frac{\sum_{k=1}^{L} o_{k}^{l} \overline{\boldsymbol{q}}_{k}^{T} \boldsymbol{\Phi}}{\sum_{k=1}^{L} o_{k}^{l}} \\
o_{k}^{r}=\frac{\sum_{k=1}^{L} o_{k}^{r}{\underline{v_{k}}}_{k}^{L} o_{k}^{r}}{\sum_{k=1}^{L} o_{k}^{r} \underline{\boldsymbol{q}}_{k}^{T} \boldsymbol{\Phi}}
\end{array}
$$

where $a_{k}^{l}, a_{k}^{r}, o_{k}^{l}$ and $o_{k}^{r}$ are calculated by the KM algorithm (Mendel 2011), and $R_{p}$ and $L_{p}$ are the right and left switch points

$$
\begin{aligned}
& a_{k}^{l}= \begin{cases}\bar{a}_{k}, & k \leq L_{p} \\
\underline{a}_{k}, & k>L_{p}\end{cases} \\
& a_{k}^{r}= \begin{cases}\underline{a}_{k}, & k \leq R_{p} \\
\bar{a}_{k}, & k>R_{p}\end{cases} \\
& o_{k}^{l}= \begin{cases}\bar{o}_{k}, & k \leq L_{p} \\
\underline{o}_{k}, & k>L_{p}\end{cases} \\
& o_{k}^{r}= \begin{cases}\underline{o}_{k}, & k \leq R_{p} \\
\underline{o}_{k}, & k>R_{p}\end{cases} \\
& a_{k}=\frac{a_{k}^{l}+a_{k}^{r}}{2} \\
& o_{k}=\frac{o_{k}^{l}+o_{k}^{r}}{2} \\
& A_{k}=a_{k} z_{k}^{a}
\end{aligned}
$$

where $z_{k}^{a}$ is the $k$ th output weight of amygdala and $A_{k}$ is the $k$ th amygdala output.

$$
O_{k}=o_{k} z_{k}^{o}
$$


where $z_{k}^{o}$ is the $k$ th output weight of orbitofrontal cortex and $O_{k}$ is the $k$ th orbitofrontal output.

\section{Layer 5: Output layer}

The proposed IT2FFFLBC is shown in Fig. 2, which is determined as:

$\boldsymbol{u}_{\text {IT } 2 F D F L E C}=\boldsymbol{A}-\boldsymbol{O}=\boldsymbol{a} \boldsymbol{z}^{a}-\boldsymbol{o z}{ }^{o}$

where $\boldsymbol{u}_{W T 2 F D F L E C C}=\left[u_{1} \mathrm{~K} u_{k} \mathrm{~K} u_{L}\right], \quad \boldsymbol{z}^{a}=\left[z_{1}^{a}, \mathrm{~K}, \mathrm{z}_{k}^{a} \mathrm{~K}, \mathrm{z}_{L}^{a}\right], \quad$ and $\quad \boldsymbol{z}^{o}=\left[z_{1}^{o}, \mathrm{~K}, \mathrm{z}_{k}^{o} \mathrm{~K}, \mathrm{z}_{L}^{o}\right]$, $\boldsymbol{A}=\left[a_{1} z_{1}^{a}, \mathrm{~K}, a_{k} z_{k}^{a} \mathrm{~K}, a_{L} z_{L}^{a}\right]^{T}$ and $\boldsymbol{O}=\left[o_{1} z_{1}^{o}, \mathrm{~K}, o_{k} z_{k}^{o} \mathrm{~K}, o_{L} z_{L}^{o}\right]^{T}$.

\section{Online Learning Laws and Convergence Analysis}

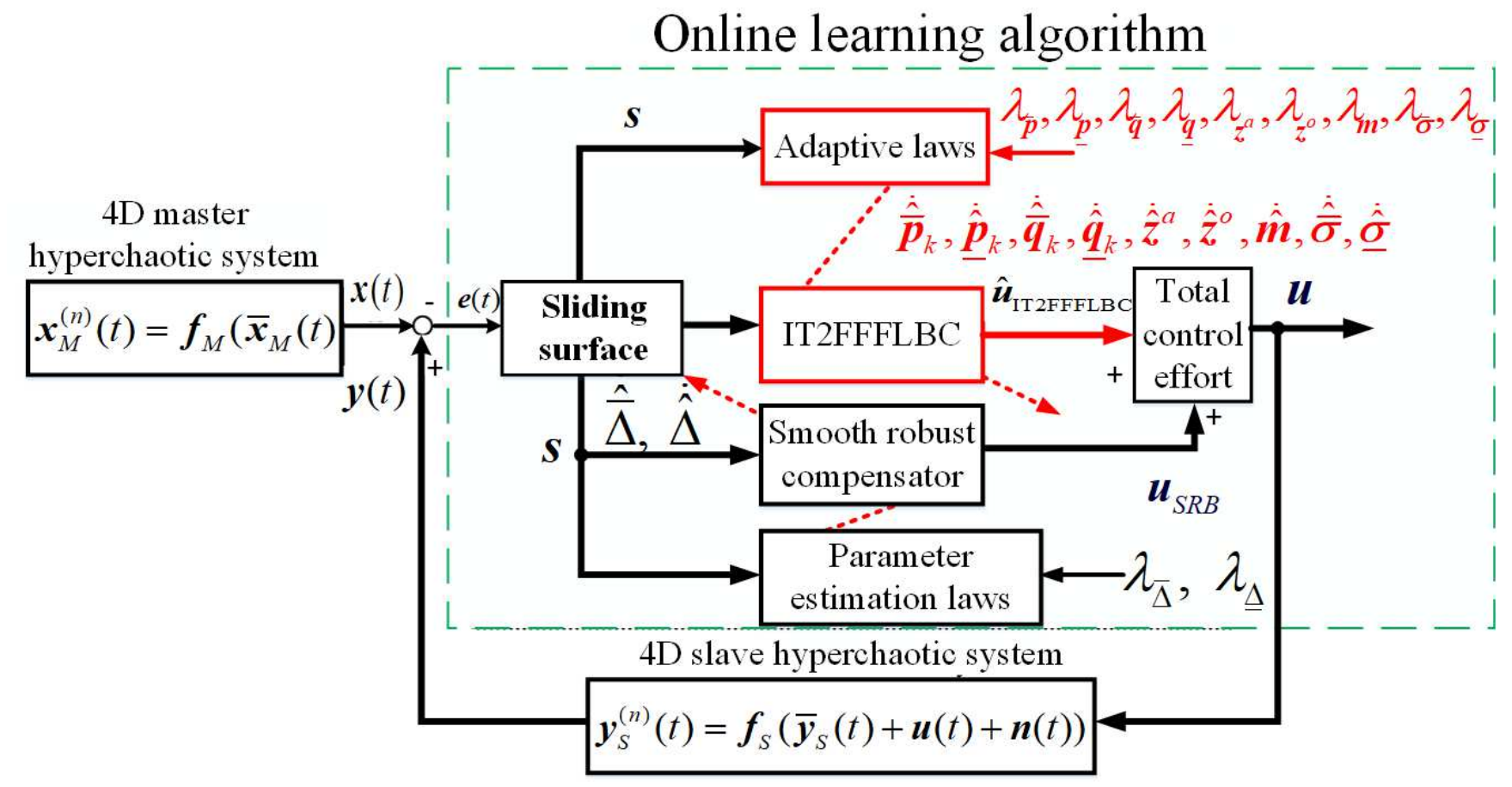

Fig. 3. Block diagram of synchronization for 4D nonlinear hyperchaotic systems using the proposed

\section{IT2FFFLBC}

The ideal controller in (5) theoretically makes the system stable. However, the nonlinear functions $\boldsymbol{f}_{M S}\left(\overline{\boldsymbol{x}}_{M S}(t)\right), \boldsymbol{f}_{S S}\left(\overline{\boldsymbol{y}}_{S S}(t)\right)$ and the external noise $\boldsymbol{n}_{S S}(t)$ are unobtainable accurately in general. As a result, (5) is unobtainable, so the proposed IT2FFFLBC is used for 4D nonlinear hyperchaotic systems as presented in Fig. 3. The total control effort is defined as:

$\boldsymbol{u}=\boldsymbol{u}_{\mathrm{IT} 2 \mathrm{FFLBC}}+\boldsymbol{u}_{\mathrm{SRC}}$

where $\boldsymbol{u}_{\mathrm{IT2FDFLC}}$ is the main controller that imitates $\boldsymbol{u}_{I D C}^{*} \cdot \boldsymbol{u}_{S R C}$ is a smooth robust compensator which spurns the dissimilarity between $\boldsymbol{u}_{I D C}^{*}$ and the proposed IT2FFFLBC. Suppose that we obtain an optimal 
controller, $\boldsymbol{u}_{\mathrm{IT} 2 \mathrm{FFFLBC}}^{*}$, that mimics an ideal controller $\boldsymbol{u}_{I D C}^{*}$ in (5), then

$\boldsymbol{u}_{\mathrm{IDC}}^{*}=\boldsymbol{u}_{\mathrm{IT} 2 \mathrm{FFFLBC}}^{*}+\boldsymbol{\varepsilon} \equiv \boldsymbol{a}^{*} \boldsymbol{z}^{a^{*}}-\boldsymbol{o}^{*} \boldsymbol{z}^{o^{*}}+\boldsymbol{\varepsilon}$

where $\boldsymbol{\varepsilon}$ is the minimum error between $\boldsymbol{u}_{I D C}^{*}$ and $\boldsymbol{u}_{\mathrm{IT} 2 \mathrm{FFFBC}}^{*}$, and assume it is bounded; $\boldsymbol{a}^{*}, \boldsymbol{o}^{*}, z^{a^{*}}$ and $z^{o^{*}}$ are respectively optimal parameters of $\boldsymbol{a}, \boldsymbol{o}, z^{a}$ and $z^{o}$. However, $\boldsymbol{u}_{\mathrm{IT} 2 \mathrm{FDFBC}}^{*}$ cannot be obtained, thus an online estimation of IT2FFFLBC, $\hat{\boldsymbol{u}}_{\text {IT2FFFLBC }}$, is applied to estimate $\boldsymbol{u}_{\text {IT2FFFBC }}^{*}$. Using (43), the control law (44) becomes

$\boldsymbol{u}=\hat{\boldsymbol{u}}_{\mathrm{IT} 2 \mathrm{FFFLC}}+\boldsymbol{u}_{\mathrm{SRC}} \equiv \hat{\boldsymbol{a}} \hat{\boldsymbol{z}}^{a}-\hat{\boldsymbol{o}} \hat{\boldsymbol{z}}^{o}+\boldsymbol{u}_{\mathrm{SRC}}$

where $\hat{\boldsymbol{a}}, \hat{\boldsymbol{o}}, \hat{z}^{a}$ and $\hat{z}^{o}$ are respectively estimations of the optimal parameters $\boldsymbol{a}^{*}, \boldsymbol{o}^{*}, \boldsymbol{z}^{a^{*}}$ and $\boldsymbol{z}^{o^{*}}$. The estimation error, $\boldsymbol{W}$, is then calculated by subtracting (46) from (45):

$\mathscr{H}=\boldsymbol{u}_{I D C}^{*}-\boldsymbol{u}=\not g^{T} \boldsymbol{a}^{*}+\hat{\boldsymbol{z}}^{a T} \not \boldsymbol{\theta} \sigma-\not 0^{T} \boldsymbol{o}^{*}-\hat{\boldsymbol{z}}^{o T} \boldsymbol{\partial} \boldsymbol{\theta} \boldsymbol{\varepsilon}-\boldsymbol{u}_{S R C}$

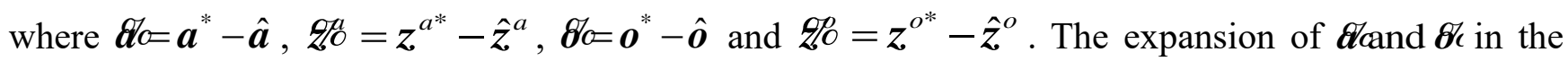
Taylor series is attained as (Slotine and Li 1991):

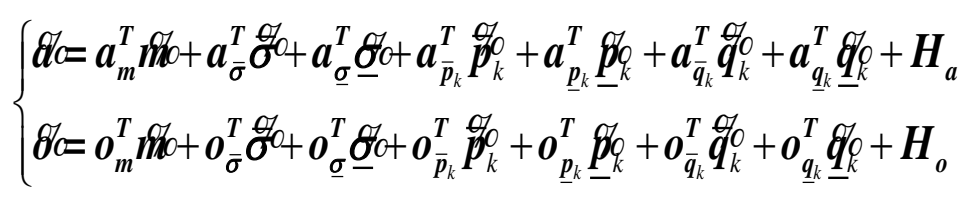

where $\boldsymbol{H}_{\boldsymbol{a}}$ and $\boldsymbol{H}_{\boldsymbol{o}} \in \mathfrak{R}^{n_{H}}$ are vectors with high-order terms, and $\frac{\partial a_{k}}{\partial \boldsymbol{m}}, \frac{\partial a_{k}}{\partial \overline{\boldsymbol{\sigma}}}, \frac{\partial a_{k}}{\partial \underline{\boldsymbol{\sigma}}}, \frac{\partial a_{k}}{\partial \overline{\boldsymbol{p}}_{k}}, \frac{\partial a_{k}}{\partial \underline{\boldsymbol{p}}_{k}}, \frac{\partial a_{k}}{\partial \overline{\boldsymbol{q}}_{k}}$, $\frac{\partial a_{k}}{\partial \underline{\boldsymbol{q}}_{k}}, \frac{\partial o_{k}}{\partial \boldsymbol{m}}, \frac{\partial o_{k}}{\partial \overline{\boldsymbol{\sigma}}}, \frac{\partial o_{k}}{\partial \underline{\boldsymbol{\sigma}}}, \frac{\partial o_{k}}{\partial \overline{\boldsymbol{p}}_{k}}, \frac{\partial o_{k}}{\partial \underline{\boldsymbol{p}}_{k}}, \frac{\partial o_{k}}{\partial \overline{\boldsymbol{q}}_{k}}$ and $\frac{\partial \boldsymbol{o}_{k}}{\partial \underline{\boldsymbol{q}}_{k}}$ are determined as:

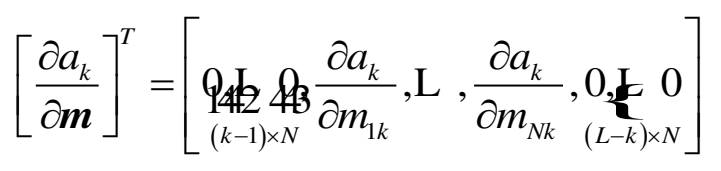

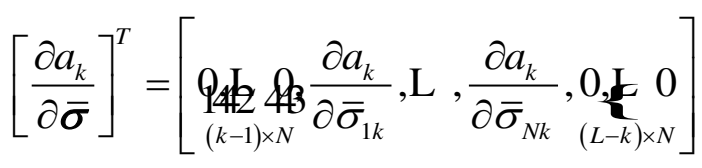

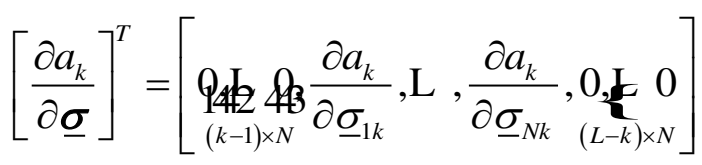

$\left[\frac{\partial a_{k}}{\partial \overline{\boldsymbol{p}}_{k}}\right]^{T}=\left[\underset{(k-1) \times M}{\operatorname{q4}_{4} \& \frac{\partial a_{k}}{\partial \bar{p}_{1 k}}, \mathrm{~L}, \frac{\partial a_{k}}{\partial \bar{p}_{M k}}, \underset{(L-k) \times M}{0, \mathrm{~L}} 0}\right]$ 


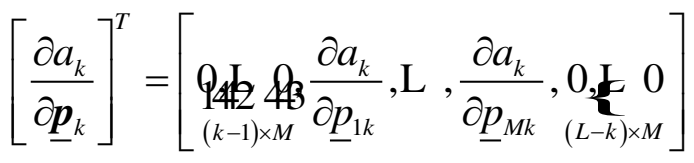

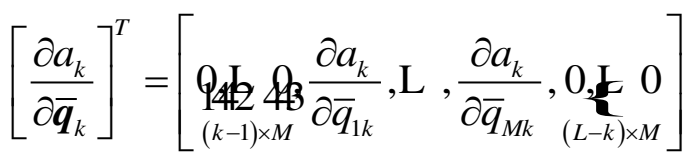

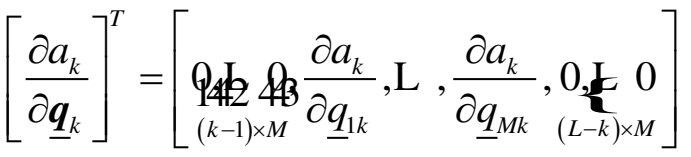

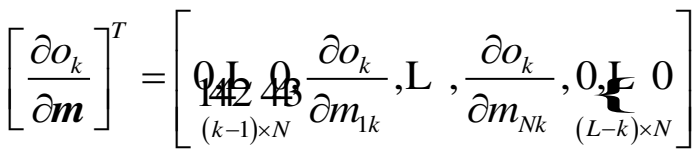

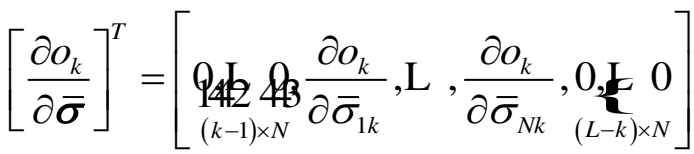

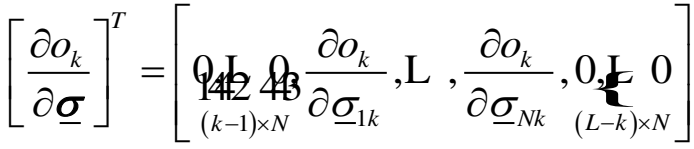

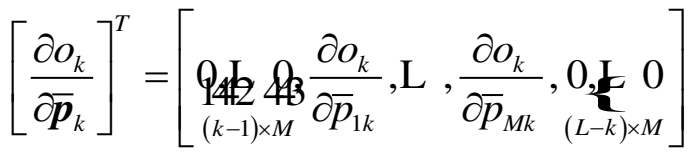

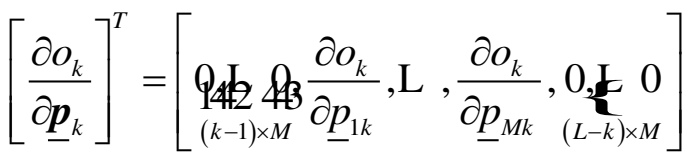

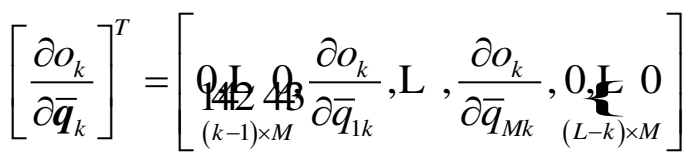

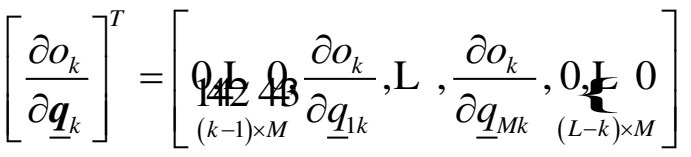

Rewriting (48) gives

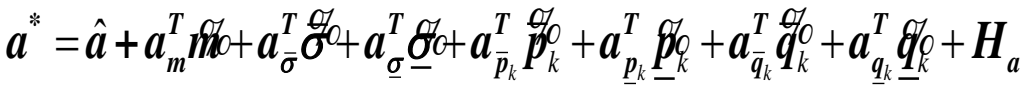

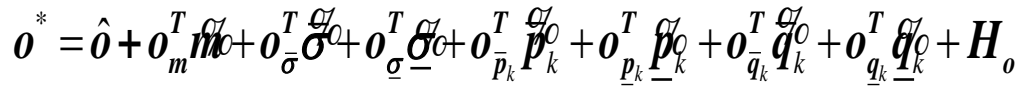

Inserting (48), (63) and (64) into (47), yields 


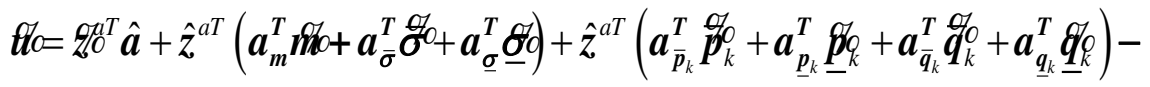

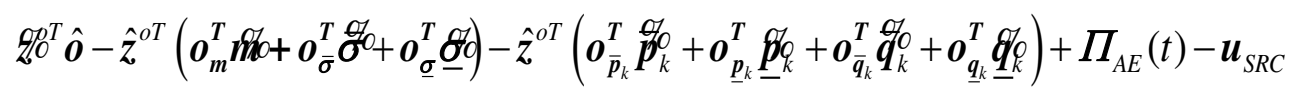

where the approximation error,

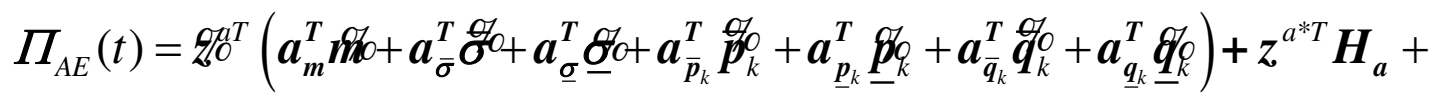

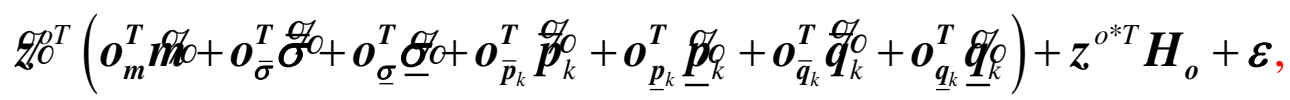

is supposedly bounded by $\left|\Pi_{A E}(t)\right|<\bar{\Delta}^{*}$, where $\bar{\Delta}^{*}$ is a positive constant.

This research uses the high-order sliding mode for improving the control system. Define the sliding surface as:

$\boldsymbol{S}(t) @ \boldsymbol{K}_{1} \boldsymbol{e}_{S y n c}+\boldsymbol{K}_{2} \boldsymbol{e}_{S y n c}^{(1)}+\mathrm{K}+\boldsymbol{K}_{n-1} \boldsymbol{e}_{S y n c}^{(n-3)}+\boldsymbol{K}_{n} \boldsymbol{e}_{S y n c}^{(n-2)}+\boldsymbol{e}_{S y n c}^{(n-1)}$

Then, taking the derivative of (66), gives

$$
\begin{aligned}
\boldsymbol{s}(t(t) & =\boldsymbol{K}_{1} \boldsymbol{e}_{S y n c}^{\mathbb{Z}}+\boldsymbol{K}_{2} \boldsymbol{e}_{S y n c}^{(2)}+\mathrm{K}+\boldsymbol{K}_{n-1} \boldsymbol{e}_{S y n c}^{(n-2)}+\boldsymbol{K}_{n} \boldsymbol{e}_{S y n c}^{(n-1)}+\boldsymbol{e}_{S y n c}^{(n)} \\
& =\boldsymbol{e}_{S y n c}^{(n)}+\overline{\boldsymbol{K}}^{T} \overline{\boldsymbol{e}}_{S S}
\end{aligned}
$$

Equation (67) is represented by using (45) and (47) as:

$$
s \&(t)=\boldsymbol{e}_{S y n c}^{(n)}+\overline{\boldsymbol{K}}^{T} \overline{\boldsymbol{e}}_{S S}=\boldsymbol{u}_{I D C}^{*}-\boldsymbol{u}
$$

Theorem 1: Consider the $n$th order 4D master-slave nonlinear hyperchaotic systems respectively given in (1) and (2). The proposed IT2FFFLBC control system is given in (44), in which $\boldsymbol{u}_{\mathrm{IT} 2 \mathrm{FFFLBC}}$ is determined in (44). The online learning laws are specified as (69)-(77) and the smooth robust compensation controller is given in (78). Then, the proposed IT2FFFLBC control system attains the robust stability.

$$
\begin{aligned}
& \underline{\boldsymbol{p}}_{k}^{\&}=\lambda_{\underline{p}} s_{k}(t)\left(\boldsymbol{a}_{\underline{p}_{k}} \hat{z}^{a}-\boldsymbol{o}_{\underline{p}_{k}} \hat{z}^{o}\right) \\
& \stackrel{\&}{\stackrel{\&}{p_{k}}}=\lambda_{\bar{p}} s_{k}(t)\left(\boldsymbol{a}_{\overline{\boldsymbol{p}}_{k}} \hat{z}^{a}-\boldsymbol{o}_{\overline{\boldsymbol{p}}_{k}} \hat{z}^{o}\right) \\
& \underline{\underline{q}}_{k}^{\&}=\lambda_{\underline{q}} s_{k}(t)\left(\boldsymbol{a}_{\underline{\boldsymbol{q}}_{k}} \hat{z}^{a}-\boldsymbol{o}_{\underline{\boldsymbol{q}}_{k}} \hat{z}^{o}\right) \\
& \stackrel{\&}{\stackrel{q}{q}}=\lambda_{\bar{q}} s_{k}(t)\left(\boldsymbol{a}_{\overline{\boldsymbol{q}}_{k}} \hat{z}^{a}-\boldsymbol{o}_{\overline{\boldsymbol{q}}_{k}} \hat{z}^{o}\right) \\
& z^{\mathfrak{k}}=\lambda_{z^{a}} s^{T}(t) \hat{a} \\
& \stackrel{\&}{\mathfrak{z}^{2}}=-\lambda_{z^{o}} \boldsymbol{s}^{T}(t) \hat{\boldsymbol{o}}
\end{aligned}
$$




$$
\begin{aligned}
& \stackrel{k}{\stackrel{k}{*}}=\lambda_{\boldsymbol{m}} \boldsymbol{s}^{T}(t)\left[\boldsymbol{a}_{\boldsymbol{m}} \hat{z}^{a}-\boldsymbol{o}_{\boldsymbol{m}} \hat{z}^{o}\right] \\
& \underline{\&}=\lambda_{\underline{\sigma}} s^{T}(t)\left[a_{\underline{\sigma}} \hat{z}^{a}-o_{\underline{\sigma}} \hat{z}^{o}\right] \\
& \stackrel{\&}{\mathscr{\sigma}}=\lambda_{\bar{\sigma}} \boldsymbol{s}^{T}(t)\left[\boldsymbol{a}_{\overline{\bar{\sigma}}} \hat{z}^{a}-\boldsymbol{o}_{\overline{\bar{\sigma}}} \hat{z}^{o}\right] \\
& \boldsymbol{u}_{S R C}=\left\{\begin{array}{l}
\hat{\bar{\Delta}} \operatorname{sgn}(\boldsymbol{s}), \text { if }|\boldsymbol{s}|>\psi \\
\underline{\hat{\Delta}} \boldsymbol{s}, \text { if }|\boldsymbol{s}| \leq \psi
\end{array}\right.
\end{aligned}
$$

where $\lambda_{\bar{p}}, \lambda_{p}, \lambda_{\bar{q}}, \lambda_{q}, \lambda_{z^{a}}, \lambda_{z^{o}}, \lambda_{m}, \lambda_{\bar{\sigma}}, \lambda_{\underline{\sigma}}$ are the positive learning rates and $\hat{\bar{\Delta}}$ is the estimated value of $\bar{\Delta}^{*}$. $\hat{\Delta}$ is an independent control parameter and $\psi$ is a positive constant, which divides the linear region between the rate of attenuation and the rate of convergence. If $\psi$ is small, the control effort is easily affected by the phenomenon of chatter. Conversely, if $\psi$ is increased to avoid chatter, the convergence speed will occur very slowly.

Proof: In case $|\boldsymbol{s}|>\psi$, the first Lyapunov function is selected as:

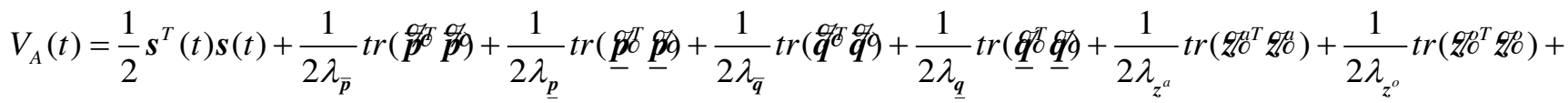

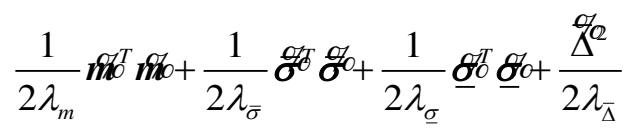

Taking the derivative of (79), then using (65) and (68), gives

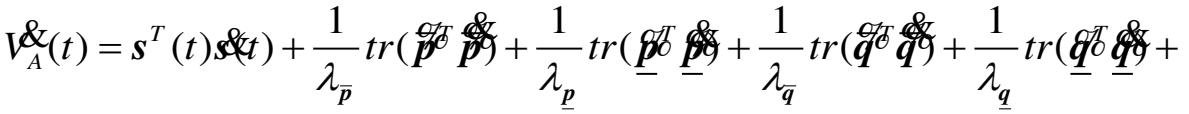

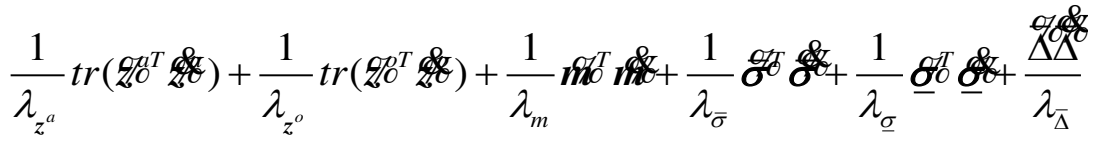

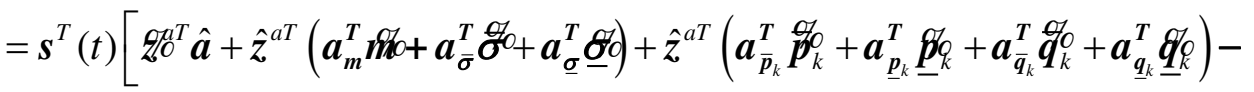

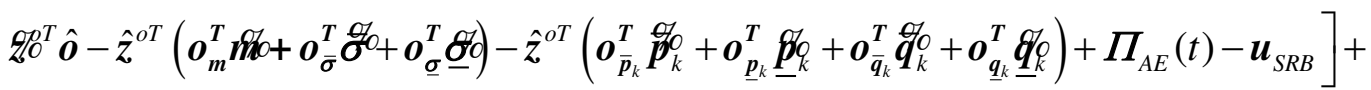

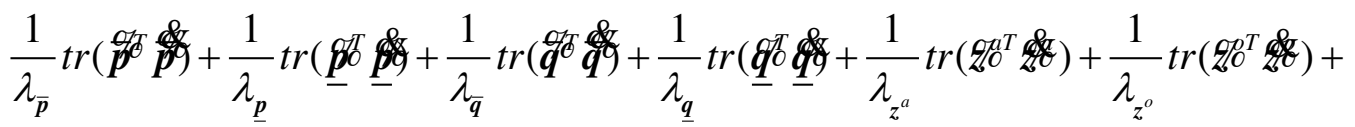

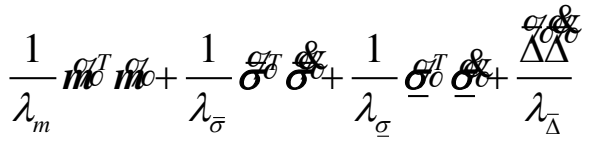




\section{Remark 1}

$\overline{\boldsymbol{p}}^{*}, \underline{\boldsymbol{p}}^{*}, \overline{\boldsymbol{q}}^{*}, \underline{\underline{q}}^{*}, z^{a^{*}}, z^{o^{*}}, \boldsymbol{m}{ }^{*}, \overline{\boldsymbol{\sigma}}^{*}$, and $\underline{\boldsymbol{\sigma}}^{*}$ are constants, thus,

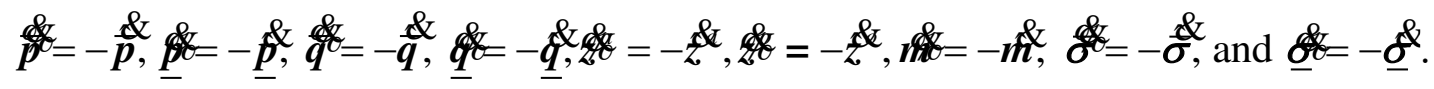

\section{$\underline{\text { Remark } 2}$}

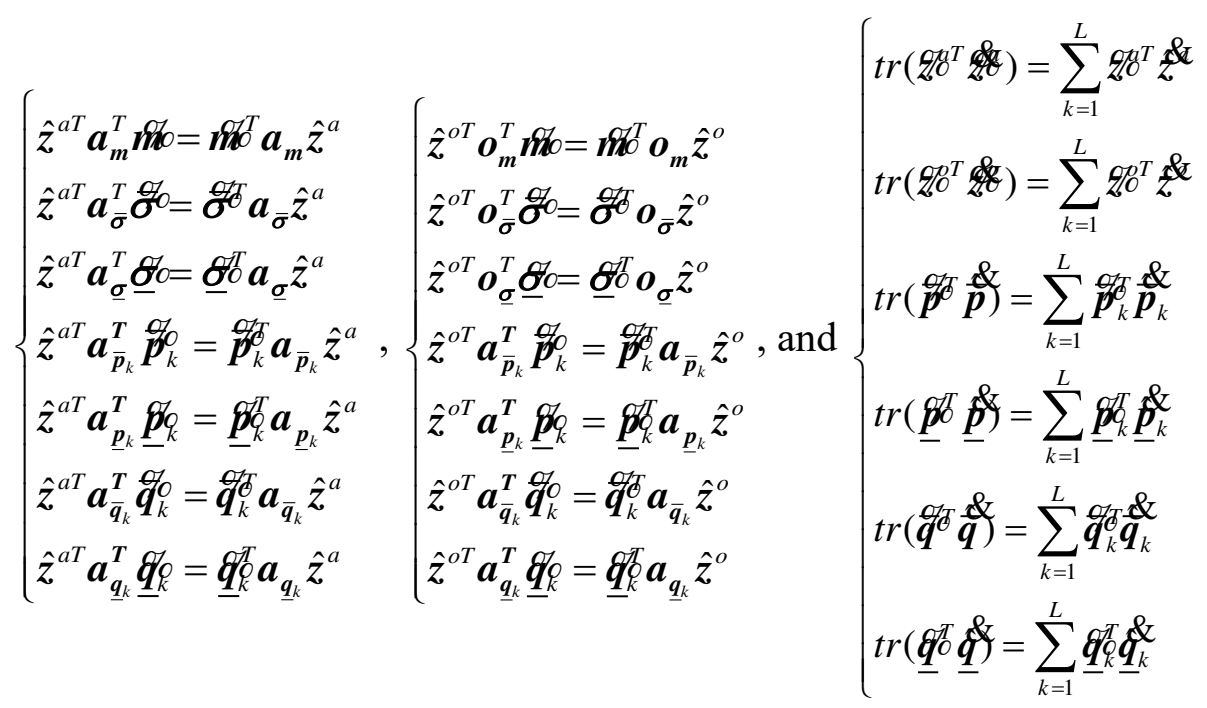

Then, (80) becomes

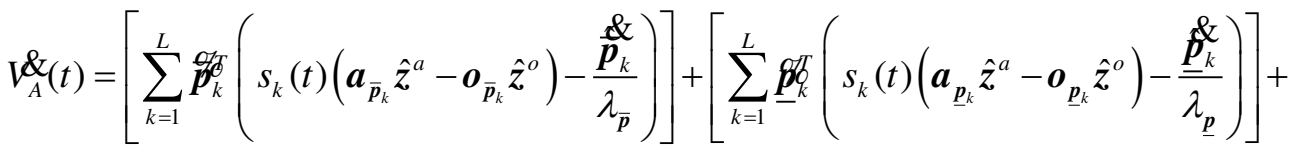

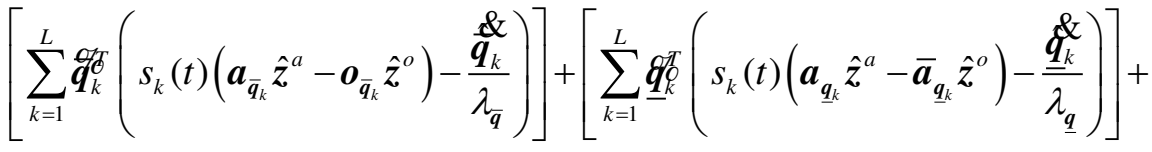

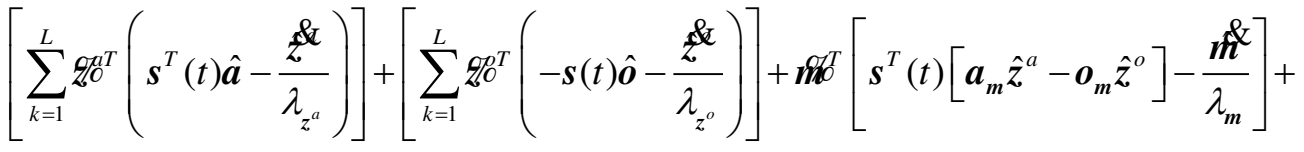

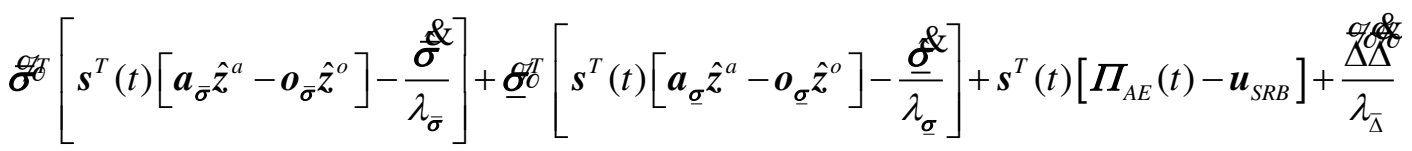

Via the adaptive laws in (69)-(77) and the robust controller in (78a), (81) is then rewritten as:

$$
\begin{aligned}
& \nu_{A}^{\&}(t)=s^{T}(t)\left[\Pi_{A E}(t)-u_{S R B}\right]+\frac{\lambda_{\bar{\Delta}}}{\lambda_{\bar{\Delta}}}=s^{T}(t)\left[\Pi_{A E}(t)-\hat{\bar{\Delta}} \operatorname{sgn}[s(t)]\right]+\frac{\hat{\Delta}}{\lambda_{\bar{\Delta}}} \\
& =s^{T}(t) \Pi_{A E}(t)-\hat{\bar{\Delta}}|s(t)|+\frac{\Delta \bar{\Delta}}{\lambda_{\bar{\Delta}}}
\end{aligned}
$$

If the error bound is updated as:

$$
\stackrel{\stackrel{\&}{\Delta}}{\Delta}=-\lambda_{\bar{\Delta}}|s(t)| \text {, }
$$


where $\lambda_{\bar{\Delta}}$ is the positive learning-rate constant, then (82) is rewritten as:

$$
\begin{aligned}
& V_{A}^{\&}(t)=\boldsymbol{s}^{T}(t) \Pi_{A E}(t)-\hat{\bar{\Delta}}|\boldsymbol{s}(t)|-(\bar{\Delta}-\hat{\bar{\Delta}})|\boldsymbol{s}(t)| \\
& \quad=\left(\boldsymbol{s}^{T}(t) \boldsymbol{\Pi}_{A E}(t)-\bar{\Delta}|\boldsymbol{s}(t)|\right) \leq\left(\left|\boldsymbol{\Pi}_{A E}(t)\right||\boldsymbol{s}(t)|-\bar{\Delta}|\boldsymbol{s}(t)|\right) \\
& \quad=-\left(\bar{\Delta}-\left|\Pi_{A E}(t)\right|\right)|\boldsymbol{s}(t)| \leq 0
\end{aligned}
$$

Since $V_{A}^{\&}(t)$ is negative semi-definite, $V_{A}^{\&}(t) \leq V_{A}^{\&}(0)$, it points out that $s(t)$ and $\mathbb{B}^{t}$ are bounded. Define $\Gamma(t) @\left(\bar{\Delta}-\left|\Pi_{A E}(t)\right|\right) s(t) \leq\left(\bar{\Delta}-\left|\Pi_{A E}(t)\right|\right)|s(t)| \leq-V_{A}^{\&}(t)$, then taking the integral $\Gamma(t)$ with respect to time, gives

$$
\int_{0}^{t} \Gamma(\tau) d \tau \leq V_{A}^{\&}(0)-V_{A}^{\&}(t)
$$

Since $V_{A}^{\&}(0)$ is bounded, and $V_{A}^{\&}(t)$ does not increase and bound, then obtain

$$
\lim _{t \rightarrow \infty} \int_{0}^{t} \Gamma(\tau) d \tau<\infty
$$

Furthermore, $\mathrm{L} \& t$ ) is bounded, then $\lim _{t \rightarrow \infty} \Gamma(t) \rightarrow 0$. It means $s \rightarrow 0$ when $t \rightarrow \infty$ (Slotine and Li 1991).

Consequently, the IT2FFFLBC control system is asymptotically stable for the case $|s|>\psi$.

The robust compensator in (78a) employs a sign(.) function to warrant the system stability. However, the robust compensation controller is normally intermittent across $s$. It implies that the control input will occur the chattering phenomenon. To prove the stability for the case $|\boldsymbol{s}| \leq \psi$, the second Lyapunov function is selected as:

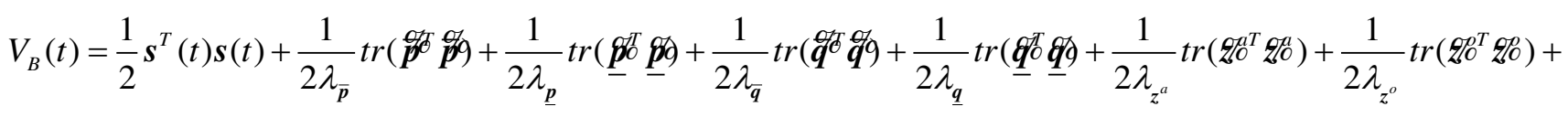

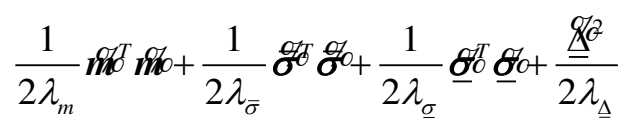

where $\lambda_{\underline{\Delta}}$ is the positive learning-rate constant.

In theory, there is an optimal constant $\underline{\Delta}^{*}$ that satisfies the robust stability for (78b) as follows:

$$
\Delta^{*}|s|>\left|\Pi_{A E}(t)\right|
$$

Taking the derivative of (87) and using (69)-(77) and (78b), gives 


$$
\begin{aligned}
& \nu_{B}^{L_{B}}(t)=\boldsymbol{s}^{T}\left[\Pi_{A E}(t)-u_{S R B}\right]+\frac{\underline{\underline{\Delta}}}{\lambda_{\underline{\Delta}}}=s^{T}\left[\Pi_{A E}(t)-\hat{\underline{\hat{\Delta}}} s\right]+\frac{\underline{\underline{\Delta}}}{\lambda_{\underline{\Delta}}} \\
& =\boldsymbol{s}^{T} \Pi_{A E}(t)-\underline{\hat{\Delta}} s^{T} s+\frac{\underline{\underline{\Delta}}}{\lambda_{\underline{\Delta}}}
\end{aligned}
$$

The parameter estimation law is selected as

$$
\underline{\leftrightarrow}=-\underline{\Delta}_{\underline{\Delta}} s^{T} s,
$$

Thus (89) becomes

$$
\begin{aligned}
& V_{B}^{\&}(t)=\boldsymbol{s}^{T} \boldsymbol{\Pi}_{A E}(t)-\underline{\hat{\Delta}} \boldsymbol{s}^{T} \boldsymbol{s}-\left(\underline{\Delta}^{*}-\underline{\hat{\Delta}}\right) \boldsymbol{s}^{T} \boldsymbol{s} \\
& \quad=\left(\boldsymbol{s}^{T} \boldsymbol{\Pi}_{A E}(t)-\underline{\Delta}^{*} \boldsymbol{s}^{T} \boldsymbol{s}\right) \leq\left(\left|\boldsymbol{\Pi}_{A E}(t)\right||\boldsymbol{s}|-\underline{\Delta}^{*}|\boldsymbol{s}||\boldsymbol{s}|\right) \\
& =-\left(\underline{\Delta}^{*}|\boldsymbol{s}|-\left|\boldsymbol{\Pi}_{A E}(t)\right|\right)|\boldsymbol{s}| \leq 0
\end{aligned}
$$

Therefore, the IT2FFFLBC control system is asymptotically stable for the case $|s| \leq \psi$. As a result of two cases, the proof is complete.

\section{Simulation Results}

This study uses two 4D nonlinear chaotic systems, a 4D hyperchaotic Lorenz-Lu system and a 4D hyperchaotic Rikitake dynamo system, to demonstrate the effectiveness and superiority of the proposed structure.

1) Chaos Synchronization for 4D Hyperchaotic Lorenz-Lu System (Chen et al. 2006; Chen et al. 2011)

The MS for the 4D hyperchaotic Lorenz's system is given as:

$$
\left\{\begin{array}{l}
\left\{(t)=a_{M S} \times\left(x_{M S 2}(t)-x_{M S 1}(t)\right)\right. \\
u_{M S 2}(t)=b_{M S} \times x_{M S 1}(t)+x_{M S 2}(t)-x_{M S 1}(t) x_{M S 3}(t)-x_{M S 4}(t) \\
x_{M S 3}(t)=x_{M S 1}(t) x_{M S 2}(t)-c_{M S} \times x_{M S 3}(t) \\
x_{M S 2}(t) x_{M S 3}(t)
\end{array}\right.
$$

The SS of the 4D hyperchaotic Lu's system is given as:

$$
\left\{\begin{array}{l}
\&_{S 1}(t)=a_{S S} \times\left(y_{S S 2}(t)-y_{S S 1}(t)\right)+y_{S S 4}(t)+u_{S S 1}(t)+n_{S S 1}(t) \\
\&_{S S 2}(t)=b_{S S} \times y_{S S 2}(t)-y_{S S 1}(t) y_{S S 3}(t)+u_{S S 2}(t)+n_{S S 2}(t) \\
\&_{S S 3}(t)=y_{S S 1}(t) y_{S S 2}(t)-c_{S S} \times y_{S S 3}(t)+u_{S S 3}(t)+n_{S S 3}(t) \\
\&_{S S 4}(t)=d_{S S} \times y_{S S 4}(t)+y_{S S 1}(t) y_{S S 3}(t)+u_{S S 4}(t)+n_{S S 4}(t)
\end{array}\right.
$$

where $x_{M S 1}(t), x_{M S 2}(t), x_{M S 3}(t), x_{M S 4}(t), y_{S S 1}(t), y_{S S 2}(t), y_{S S 3}(t)$ and $y_{S S 4}(t)$ are respectively the states of MS and SS. The parameters of the MS and SS are set as $a_{M S}=10, b_{M S}=28, c_{M S}=8 / 3, d_{M S}=0.1$, $a_{S S}=36, b_{S S}=20, c_{S S}=3, d_{S S}=1$, respectively. In this example, the external noises (see Fig. 4) are 
assumed as $\boldsymbol{n}_{S S}(t)=\left[n_{S S 1}(t), n_{S S 2}(t), n_{S S 3}(t), n_{S S 4}(t)\right]=[0.2 \times \cos (\pi t), 0.1 \times \sin (t), 0.3 \times \sin (2 t), 0.1 \times \cos (t)]$, the initial position states for the MS $x_{M S 1}(0)=-1.0, x_{M S 2}(0)=-1.0, x_{M S 3}(0)=1.0, x_{M S 4}(0)=1.0$ and the SS $y_{S S 1}(0)=5.0, y_{S S 2}(0)=2.0, y_{S S 3}(0)=-5.0, y_{S S 4}(0)=-2.0$ are used. The initial parameters for learning rates are selected as $\lambda_{\bar{p}}=\lambda_{\underline{p}}=0.2, \lambda_{\bar{q}}=\lambda_{\underline{q}}=0.1, \lambda_{z^{a}}=\lambda_{z^{o}}=0.5, \lambda_{m}=0.1, \lambda_{\bar{\sigma}}=\lambda_{\underline{\sigma}}=0.05$, and $\lambda_{\Delta}=0.5$, and $\boldsymbol{K}_{1}=3.8 \boldsymbol{I}_{4 \times 4}, \boldsymbol{K}_{2}=0.8 \boldsymbol{I}_{4 \times 4}$. The simulation results for this example are displayed in Figs. 5-17. Particularly, the 3D trajectory phase portraits of the 4D synchronization are manifested in Figs. 5-8. The 2D state trajectories are plotted in Figs. 9-15. In addition, the synchronization errors are displayed in Fig. 16, which points out that the tracking errors are quickly driven to zero. Finally, the control efforts are presented in Fig. 17. The simulation results of synchronization for the 4D hyperchaotic Lorenz-Lu system attain good performance and rapid response regardless of the influence of external noises and uncertainty parameters of the system. Moreover, the total RMSE is measured to demonstrate that the proposed IT2FFFLBC control system synchronizes the master-slave systems well with smaller tracking errors than other control systems (see Table 1). The results for the proposed IT2FFFLBC control system are also compared with some former methods such as adaptive PID (Chen et al. 2011), radial basis function neural network (RBFNN) (Chen et al. 2011), neuro-wavelet control (NWC) using integral (I)-type training method (Chen et al. 2011), NWC using (proportional-integral) PI-type training method (Chen et al. 2011). From that, we can see the IT2FFFLBC control system is qualified to handle well the noises and uncertainties than the other control systems. Table 1 points out that the proposed IT2FFFLBC control system has a smaller root mean square error (RMSE) and attains better in synchronizing the 4D hyperchaotic Lorenz-Lu system with smaller tracking errors than other methods. Our findings indicate that the IT2FFFLBC control system can work well with the impact of external noises and uncertainties of system parameters. 

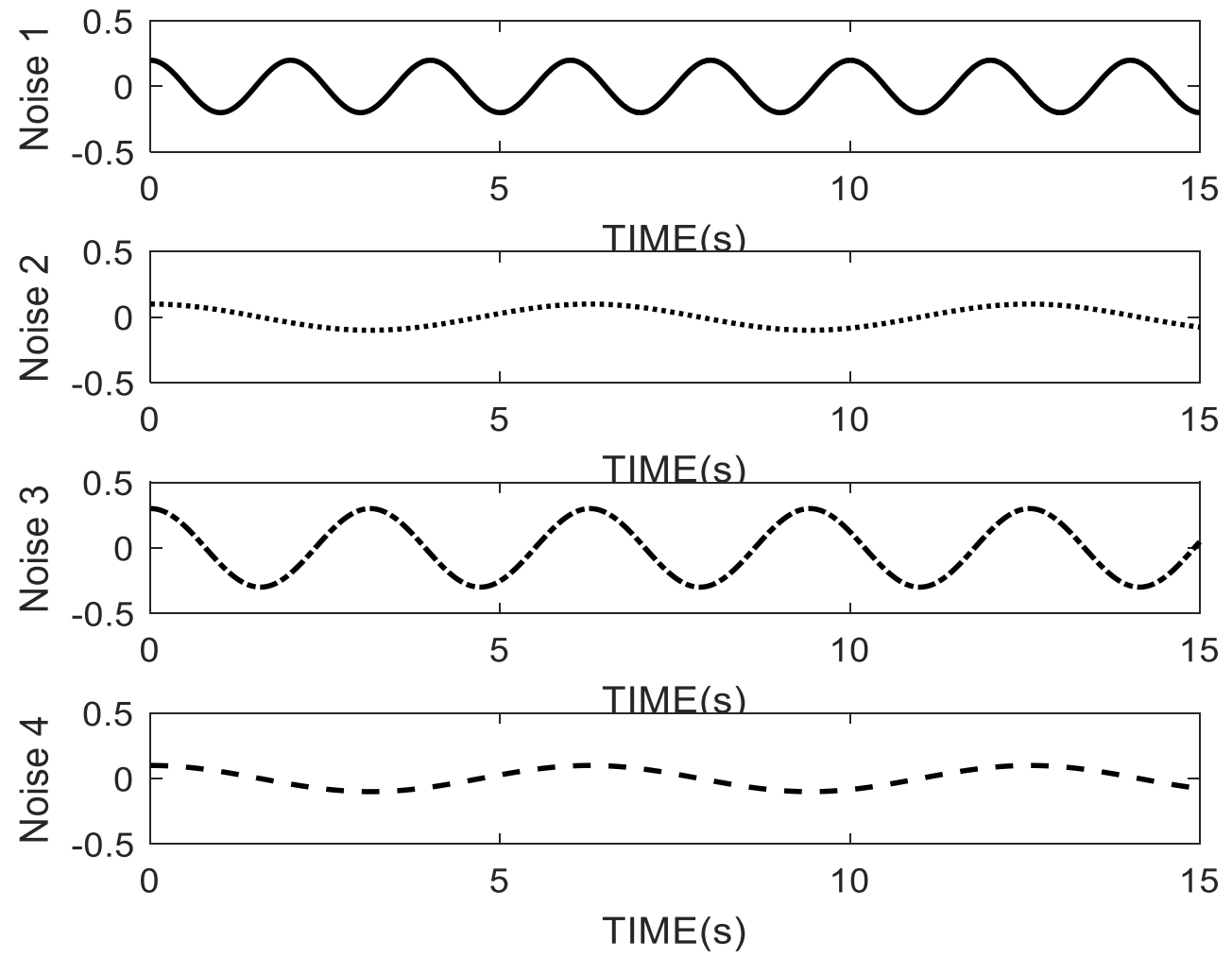

Fig. 4. External noises using for synchronization of the 4D hyperchaotic Lorenz-Lu system

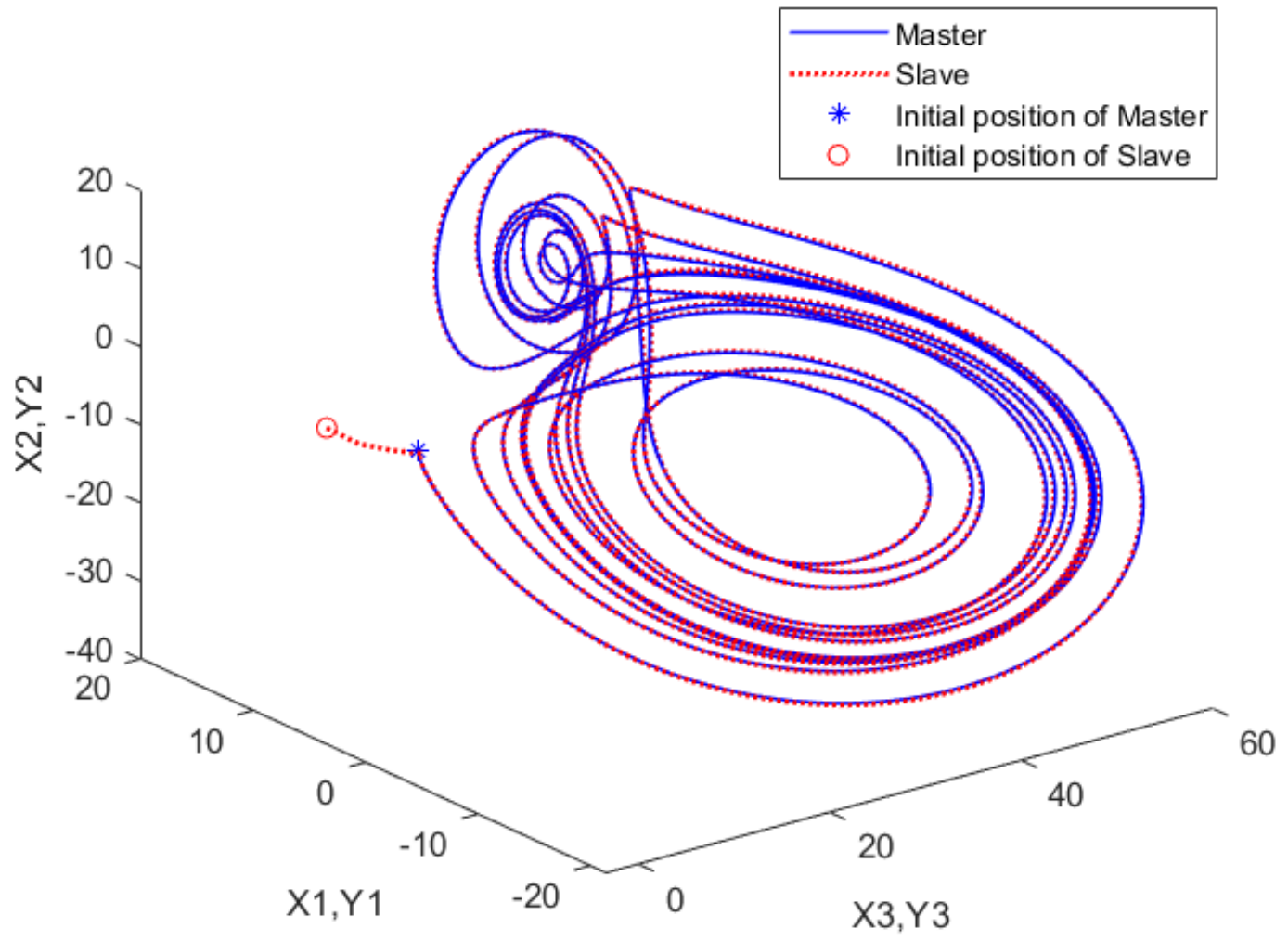

Fig. 5. 3D trajectory phase portrait of master (X1, X2, X3) and slave (Y1, Y2, Y3) 


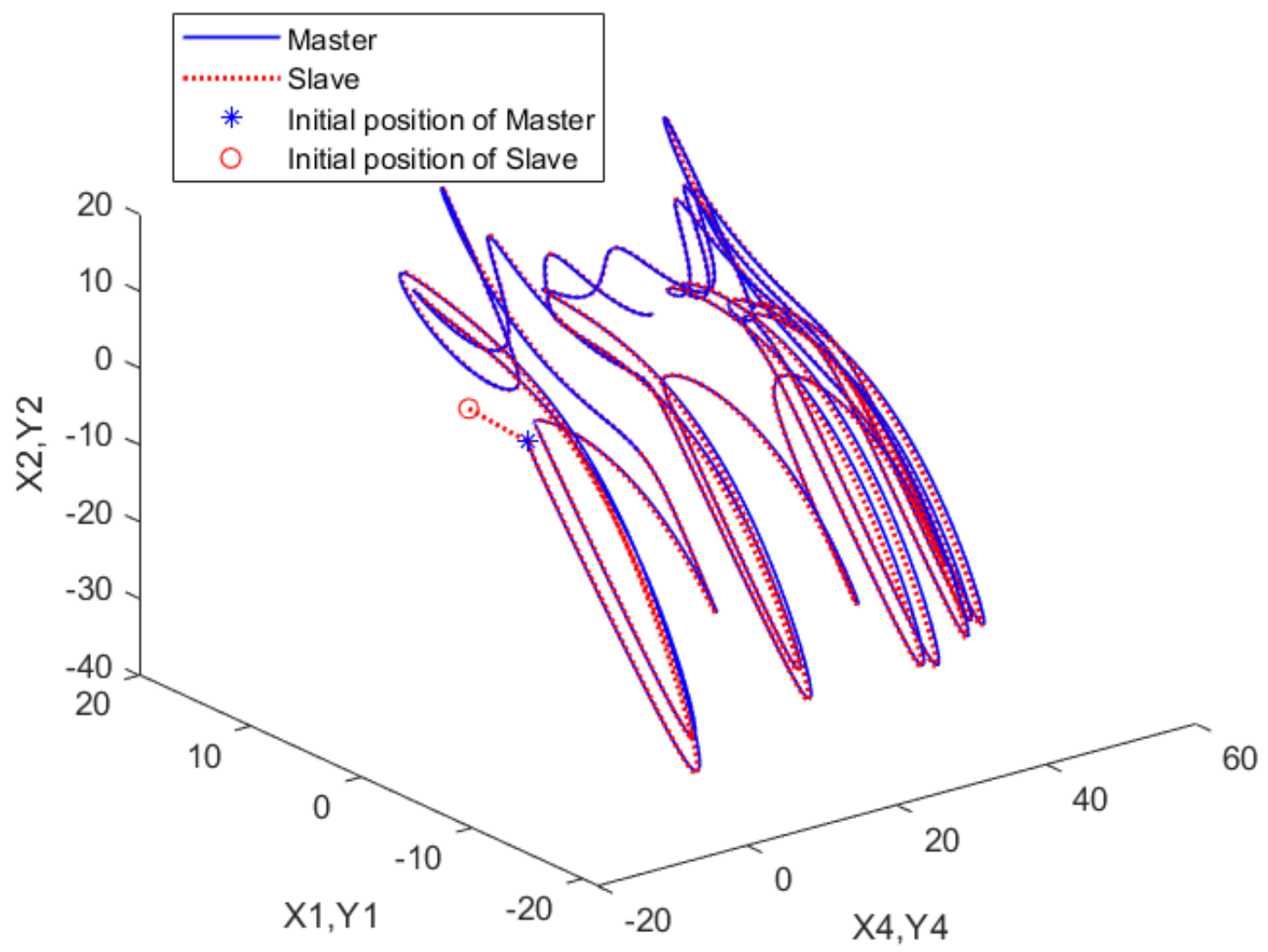

Fig. 6. 3D trajectory phase portrait of master (X1, X2, X4) and slave (Y1, Y2, Y4)

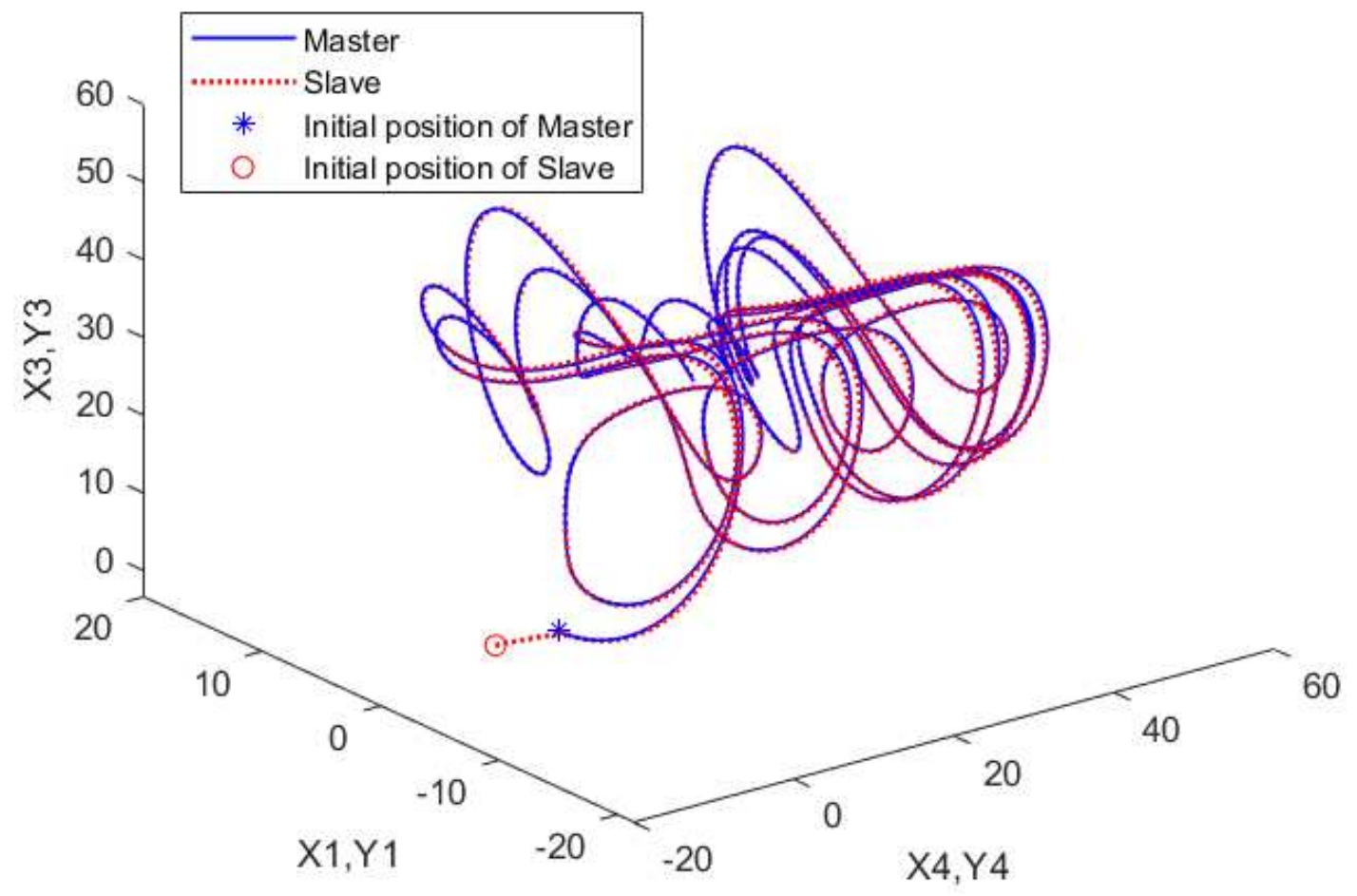

Fig. 7. 3D trajectory phase portrait of master (X1, X3, X4) and slave (Y1, Y3, Y4) 


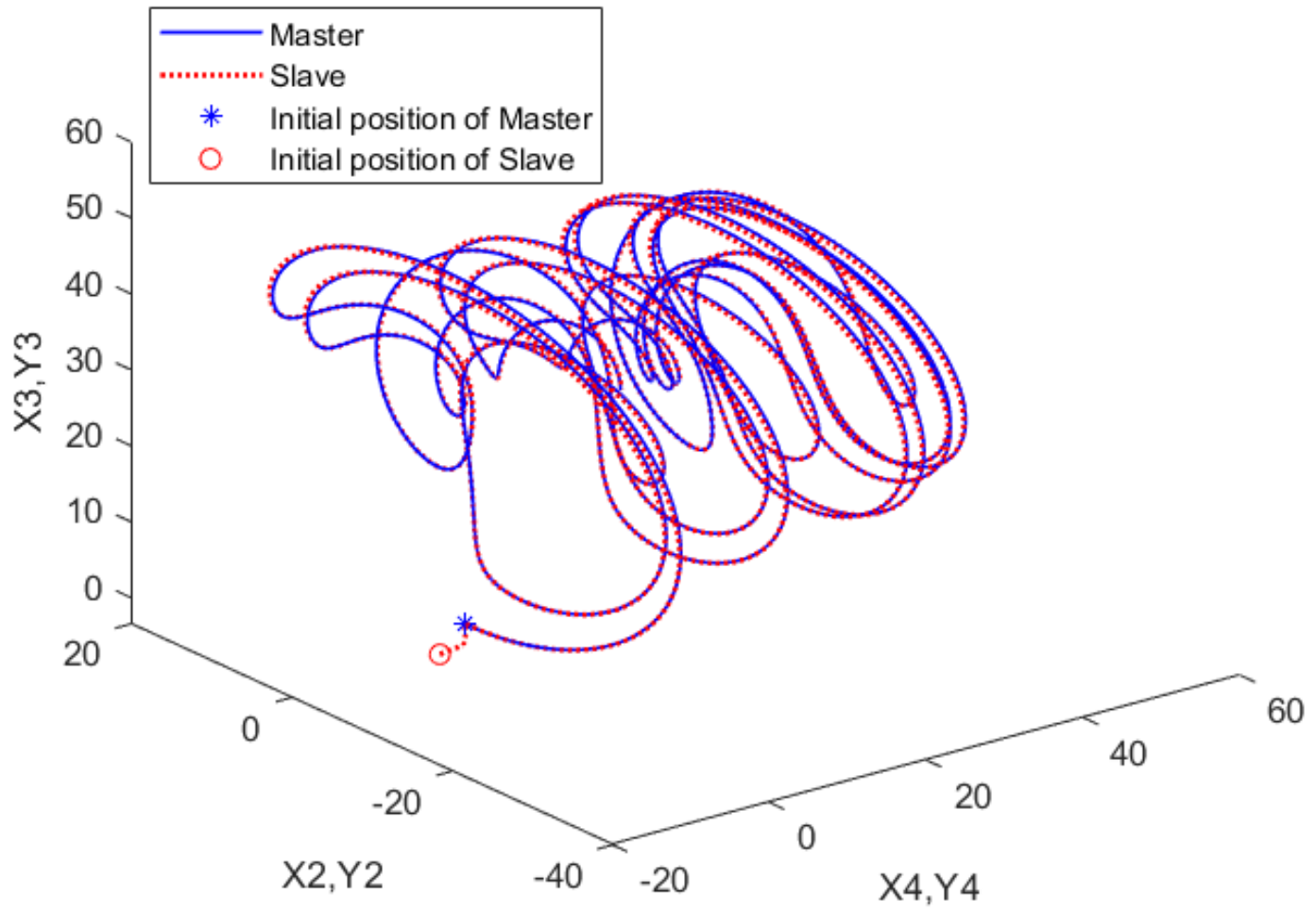

Fig. 8. 3D trajectory phase portrait of master (X2, X3, X4) and slave (Y2, Y3, Y4)

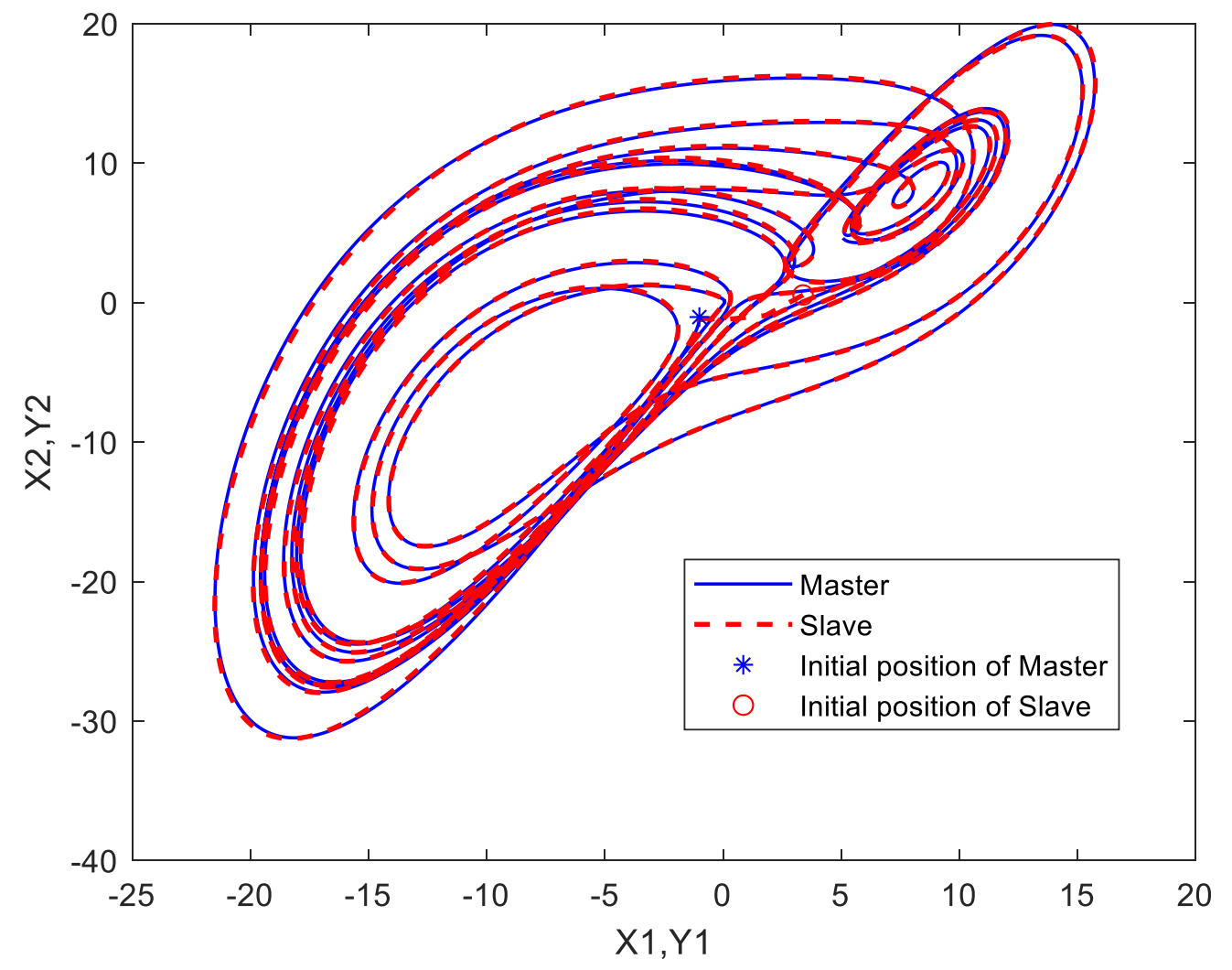

Fig. 9. The state trajectory for master $(\mathrm{X} 1, \mathrm{X} 2)$ and slave $(\mathrm{Y} 1, \mathrm{Y} 2)$ 


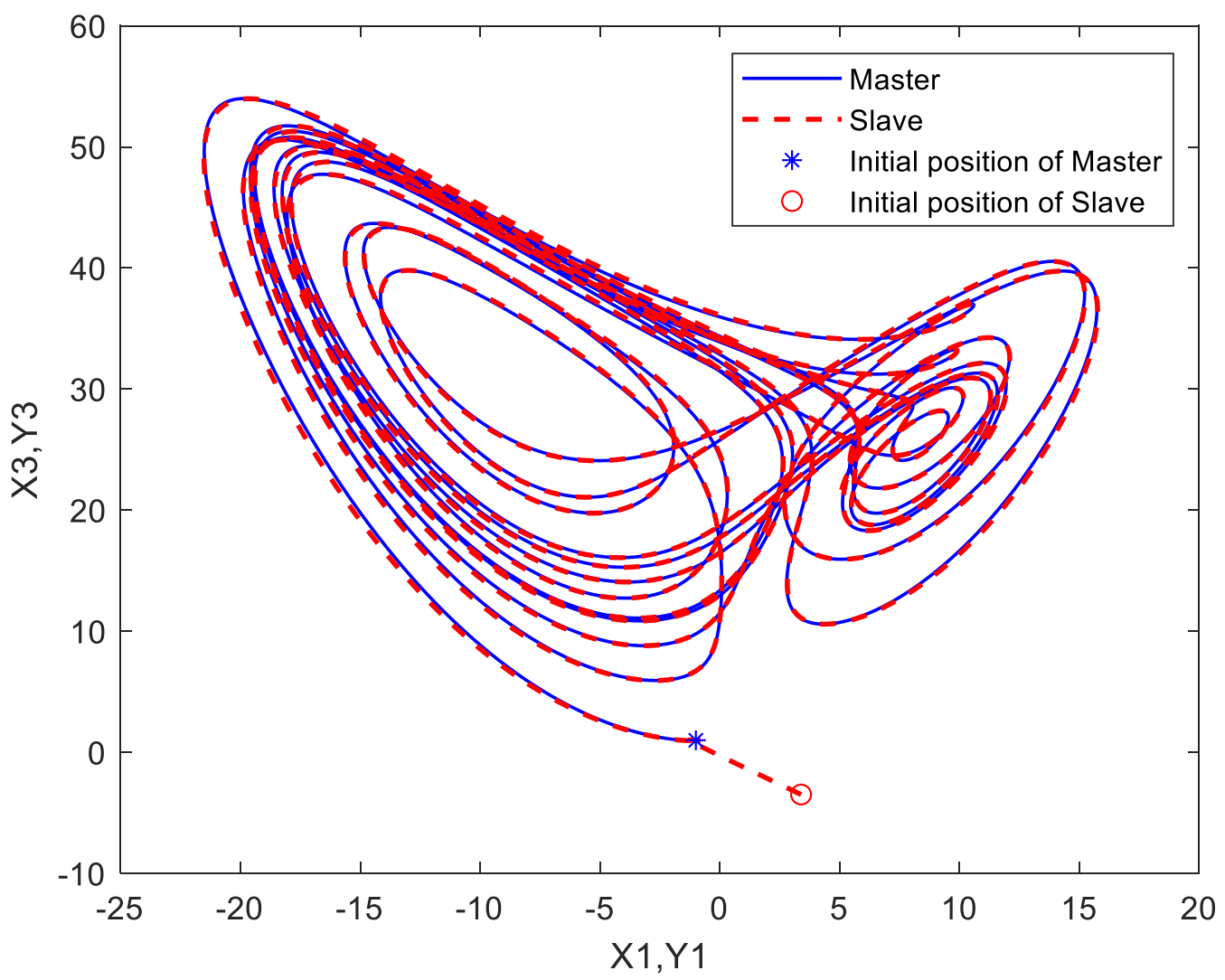

Fig. 10. The state trajectory for master $(X 1, X 3)$ and slave $(Y 1, Y 3)$

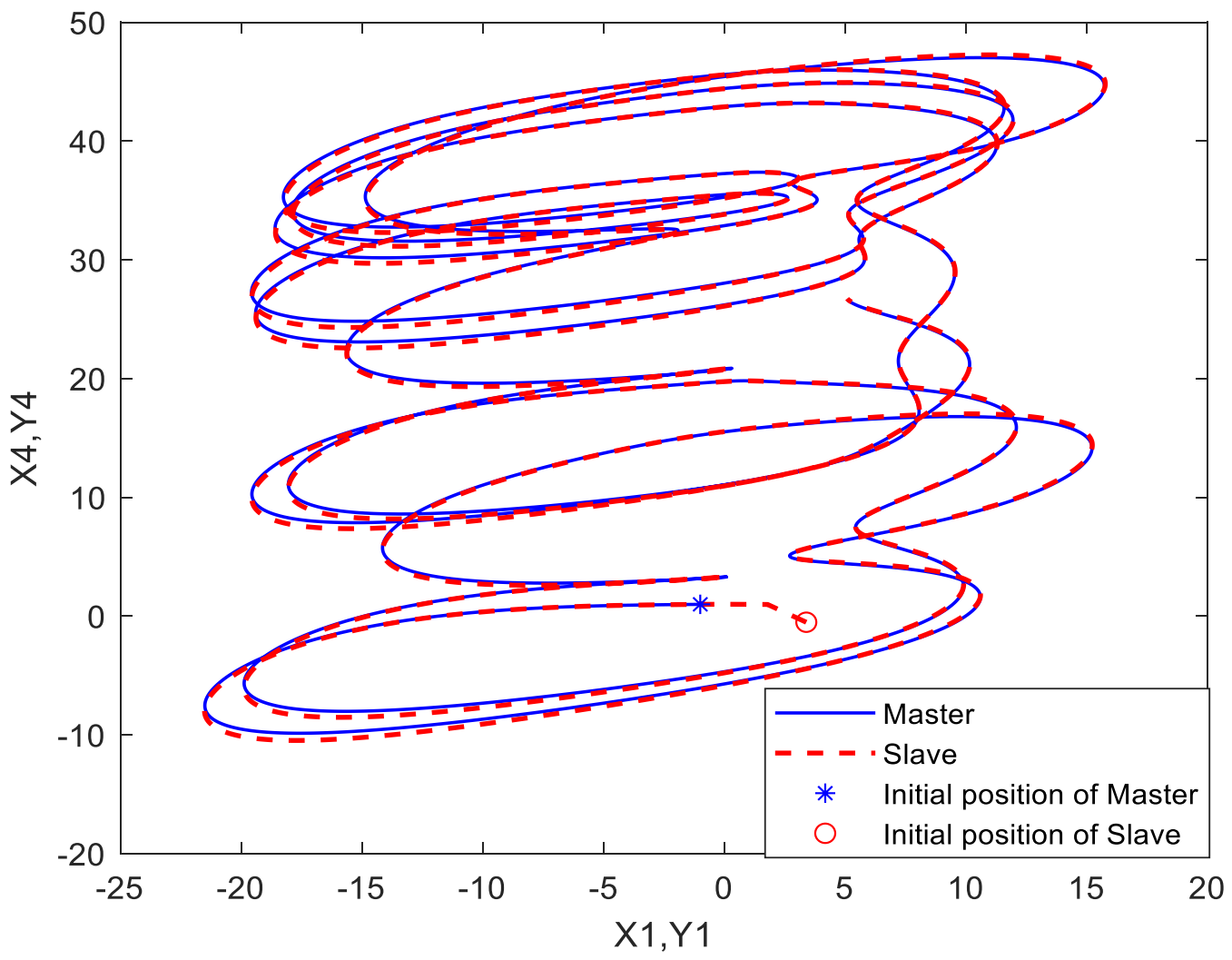

Fig. 11. The state trajectory for master (X1, X4) and slave (Y1, Y4) 


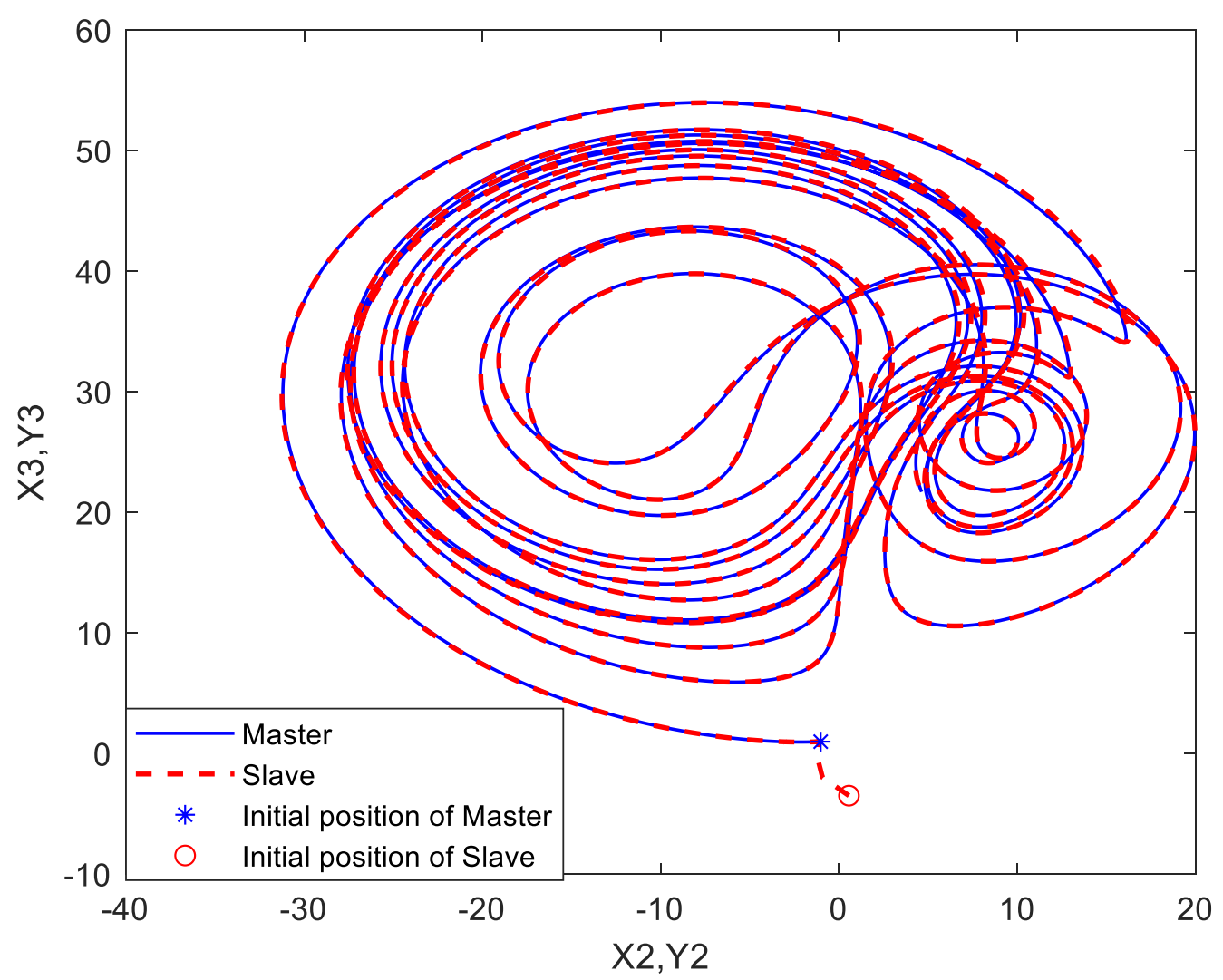

Fig. 12. The state trajectory for master (X2, X3) and slave (Y2, Y3)

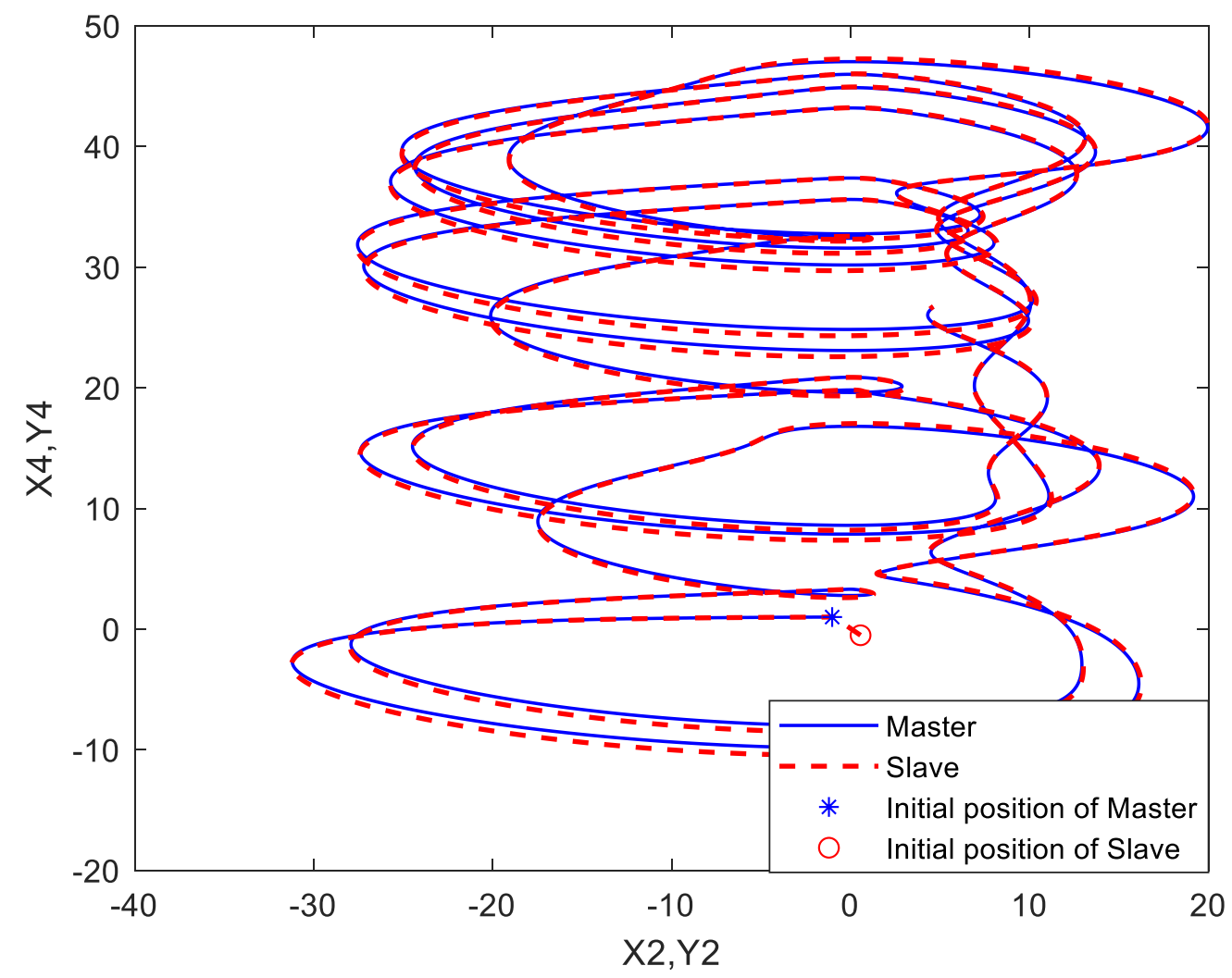

Fig. 13. The state trajectory for master $(\mathrm{X} 2, \mathrm{X} 4)$ and slave $(\mathrm{Y} 2, \mathrm{Y} 4)$ 


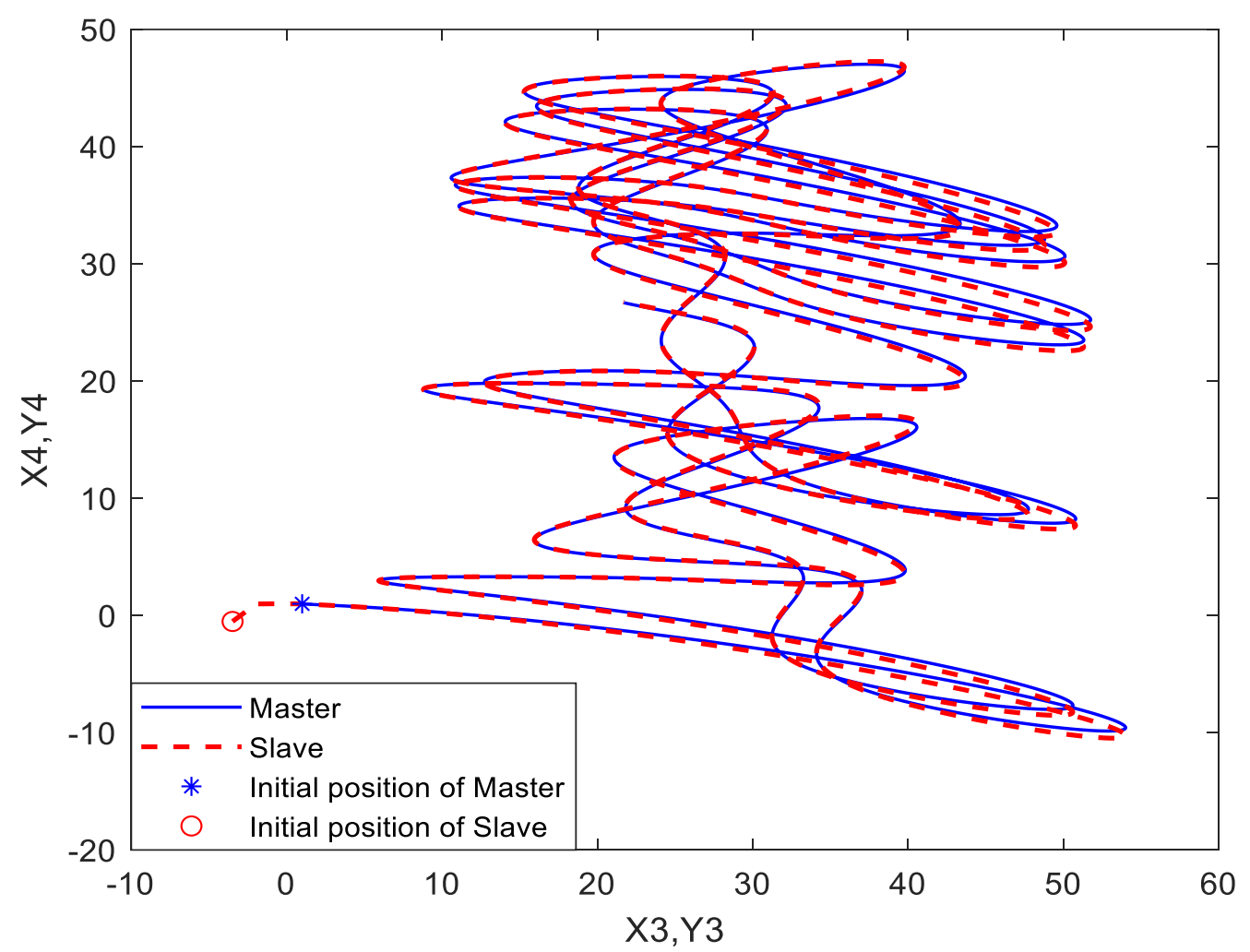

Fig. 14. The state trajectory for master $(\mathrm{X} 3, \mathrm{X} 4)$ and slave $(\mathrm{Y} 3, \mathrm{Y} 4)$
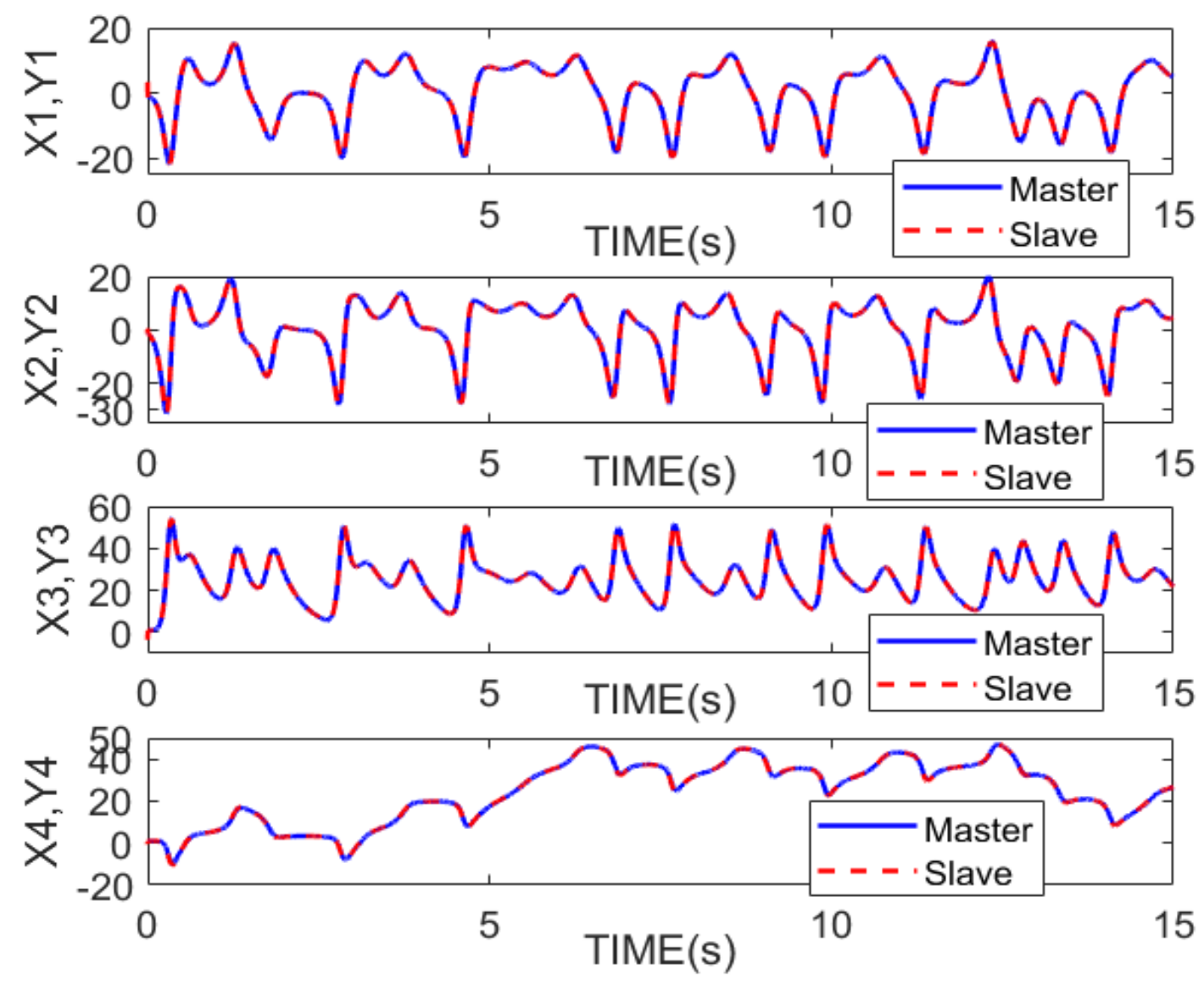

Fig. 15. State trajectories for master-slave (X1, Y1), (X2, Y2), (X3, Y3) and (X4, Y4) 

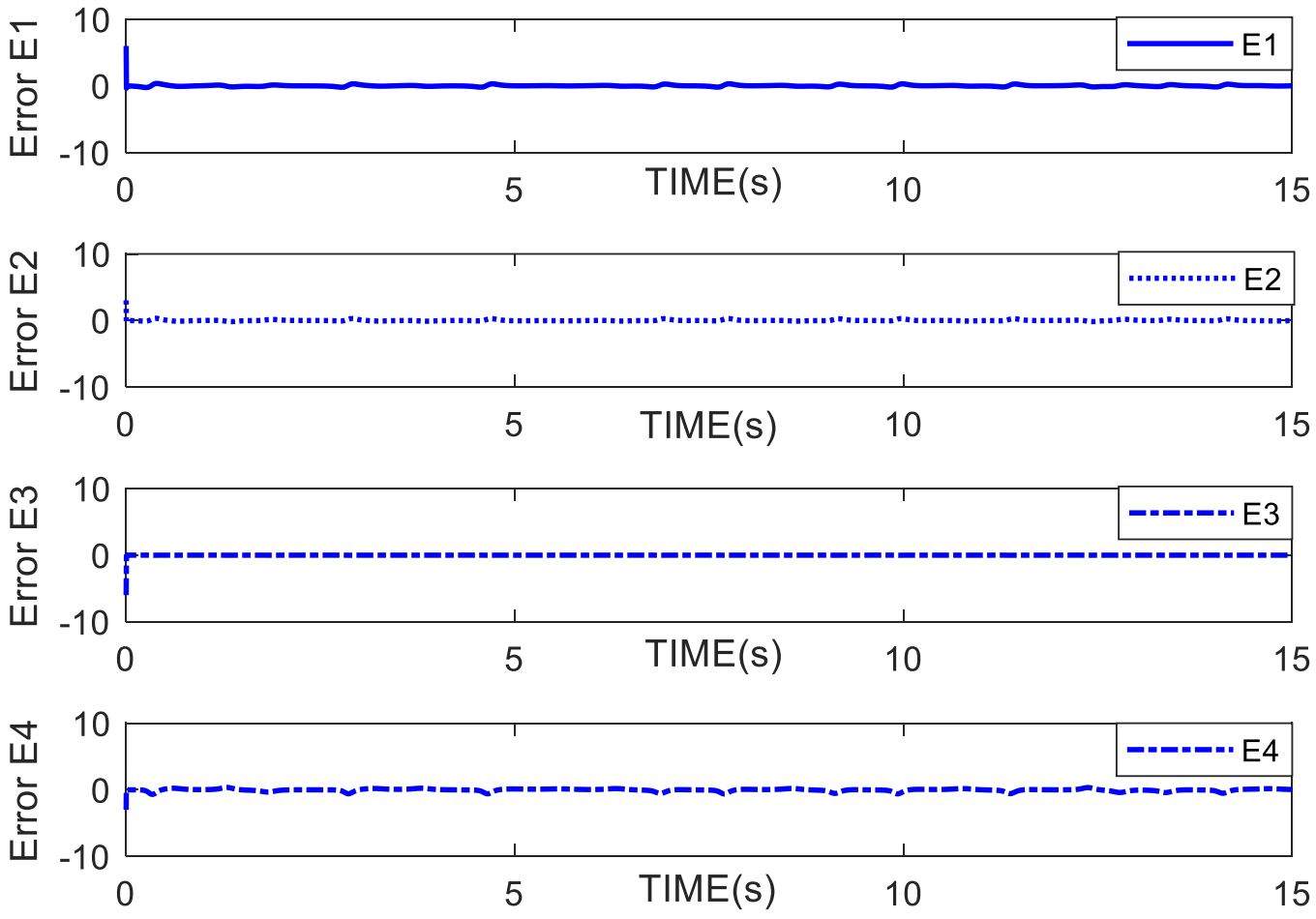

Fig. 16. Errors for synchronization of the 4D hyperchaotic Lorenz-Lu system

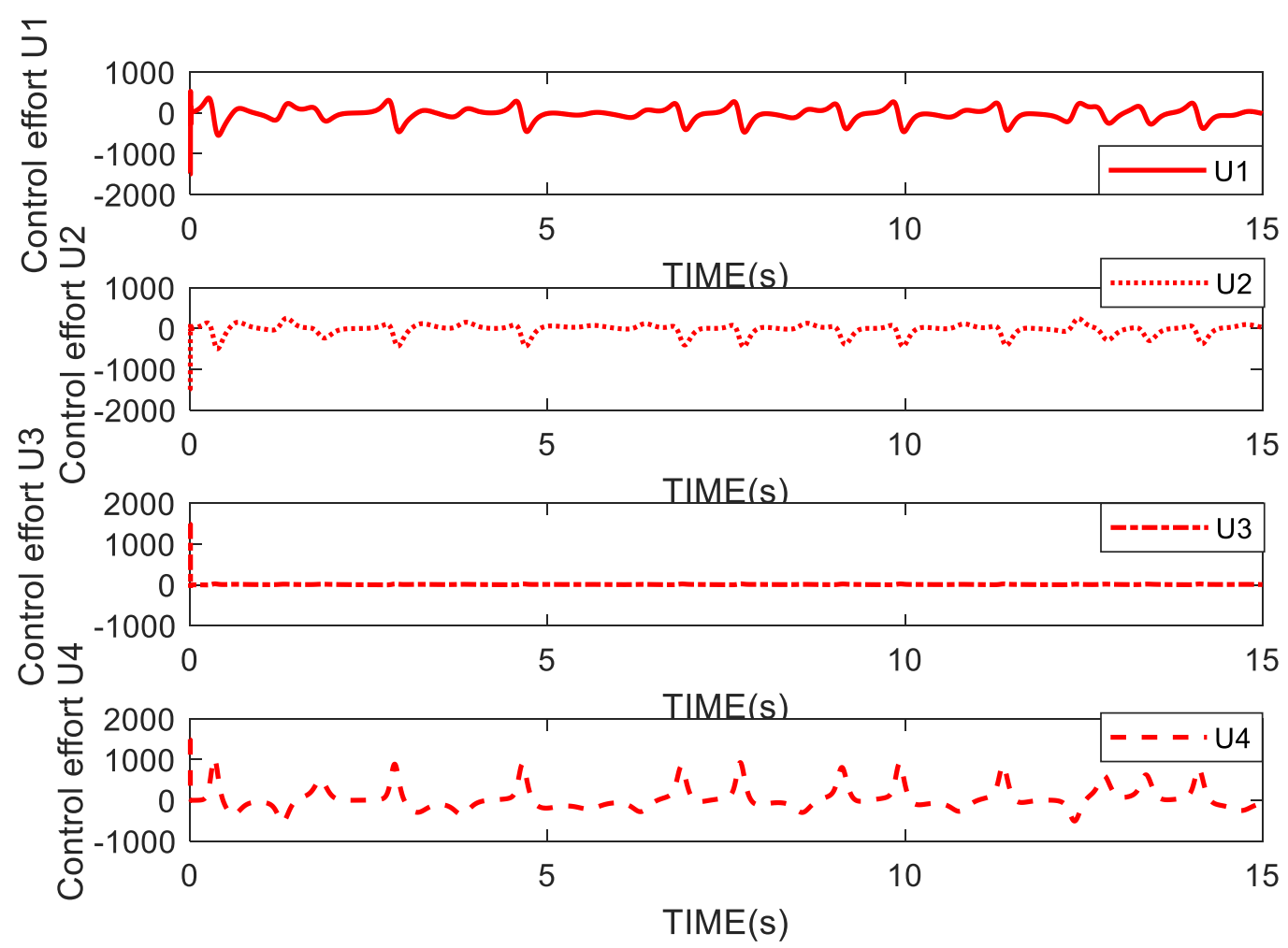

Fig. 17. Control efforts for synchronization of the 4D hyperchaotic Lorenz-Lu system 
Table 1 The comparison of RMSE between IT2FFFBC control system and the former methods

\begin{tabular}{|l|l|l|l|l|c|}
\hline & RMSE1 & RMSE2 & RMSE3 & RMSE4 & Average RMSE \\
\hline Adaptive PID & 8.2922 & 5.8457 & 1.9369 & 9.7788 & 6.4634 \\
\hline RBFNN & 2.6765 & 1.9455 & 1.0070 & 3.1995 & 2.2071 \\
\hline NWC with I-type training method & 1.5102 & 1.0407 & 0.6064 & 1.6916 & 1.2122 \\
\hline NWC with PI-type training method & 0.3828 & 0.2693 & 0.1324 & 0.4483 & 0.3082 \\
\hline IT2FFFLBC control system & $\mathbf{0 . 1 2 1 9}$ & $\mathbf{0 . 0 9 2 4}$ & $\mathbf{0 . 0 6 7 1}$ & $\mathbf{0 . 1 8 4 2}$ & $\mathbf{0 . 1 1 6 4}$ \\
\hline
\end{tabular}

\section{2)Chaos Synchronization for 4D Hyperchaotic Rikitake Two-Wing Dynamo System (Vaidyanathan} et al. 2018)

The MS is given as:

$$
\left\{\begin{array}{l}
\oiiint_{M S 1}(t)=-\alpha_{M S} \times x_{M S 1}(t)+x_{M S 2}(t) x_{M S 3}(t)-x_{M S 4}(t) \\
\underbrace{}_{M S 3}(t)=1-x_{M S 1}(t) x_{M S 2}(t) \\
x_{M S 4}(t)=\gamma_{M S} \times x_{M S 2}(t)
\end{array}\right.
$$

and, the $\mathrm{SS}$ is given as:

$$
\left\{\begin{array}{l}
\&_{S S 1}(t)=-\alpha_{S S} \times y_{S S 1}(t)+y_{S S 2}(t) y_{S S 3}(t)-y_{S S 4}(t)+u_{S S 1}(t)+n_{S S 1}(t) \\
\&_{S S 2}(t)=-\alpha_{S S} \times y_{S S 2}(t)+\left(y_{S S 3}(t)-\beta_{S S}\right) y_{S S 1}(t)-y_{S S 4}(t)+u_{S S 2}(t)+n_{S S 2}(t) \\
\&_{S S 3}(t)=1-y_{S S 1}(t) y_{S S 2}(t)+u_{S S 3}(t)+n_{S S 3}(t) \\
\&_{S S 4}(t)=\gamma_{S S} \times y_{S S 2}(t)+u_{S S 4}(t)+n_{S S 4}(t)
\end{array}\right.
$$

where $x_{M S 1}(t), x_{M S 2}(t), x_{M S 3}(t), x_{M S 4}(t), y_{S S 1}(t), y_{S S 2}(t), y_{S S 3}(t)$ and $y_{S S 4}(t)$ are the states of the MS and SS.

The parameters of the MS and SS are set as $\alpha_{M S}=\alpha_{S S}=1.0, \beta_{M S}=\beta_{S S}=1.0, \gamma_{M S}=\gamma_{S S}=0.7$. In this example, the external noises (see Fig. 18) are given as $\boldsymbol{n}_{S S}(t)=\left[n_{S S 1}(t), n_{S S 2}(t), n_{S S 3}(t), n_{S S 4}(t)\right]=$ $[0.2 \times \cos (\pi \times t), 0.1 \times \cos (t), 0.3 \times \cos (2 \times t), 0.1 \times \cos (t)]$, the initial position states of the MS $x_{M S 1}(0)=0.4, x_{M S 2}(0)=0.4, x_{M S 3}(0)=0.4, x_{M S 4}(0)=0.4 \quad$ and $\quad$ the $\quad$ SS $y_{S S 1}(0)=-1.0, y_{S S 2}(0)=-1.0, y_{S S 3}(0)=-1.0, y_{S S 4}(0)=-1.0$ are utilized. The initial parameters of the IT2FFFLBC control system are $\lambda_{\bar{p}}=\lambda_{\underline{p}}=0.5, \lambda_{\bar{q}}=\lambda_{\underline{q}}=0.1, \lambda_{z^{a}}=\lambda_{z^{o}}=0.2, \quad \lambda_{m}=0.5, \quad \lambda_{\bar{\sigma}}=\lambda_{\underline{\sigma}}=0.1$, and $\lambda_{\Delta}=0.5$. The simulation results using the proposed IT2FFFLBC control system for the 4D hyperchaotic Rikitake two-wing dynamo system are plotted in Figs. 19-31. Specifically, the 3D results are plotted in Figs. 19-22. The 2D results of state trajectories are illustrated in Figs. 23-29, respectively. Subsequent, the synchronization errors are shown in Fig. 30, which indicate that they are rapidly converged to zero. Then, the control efforts are shown in Fig. 31. At the initial, the control efforts are increased to suitable values, then they are reduced to nearly zero after achieving the synchronization. According to the simulation results, our findings point out that the synchronization of the 4D hyperchaotic Rikitake two-wing dynamo system attains good performance and quick response even with 
the impact of external noises and uncertain parameters. The results in RMSE of some recent controllers such as a recurrent cerebellar model articulation controller (RCMAC) (Huynh et al. 2020), a fuzzy brain emotional learning controller (Lin and Chung 2015), a brain-imitated neural network controller (Lin et al. 2021), and the proposed IT2FFFLBC control system are compared and shown in Table 2. The proposed IT2FFFLBC control system synchronizes well the master-slave systems with smaller tracking errors than other controllers. In summary, from the simulation results of two examples, our findings show that the IT2FFFLBC control system can work well for 4D nonlinear hyperchaotic systems with the impact of external noises.
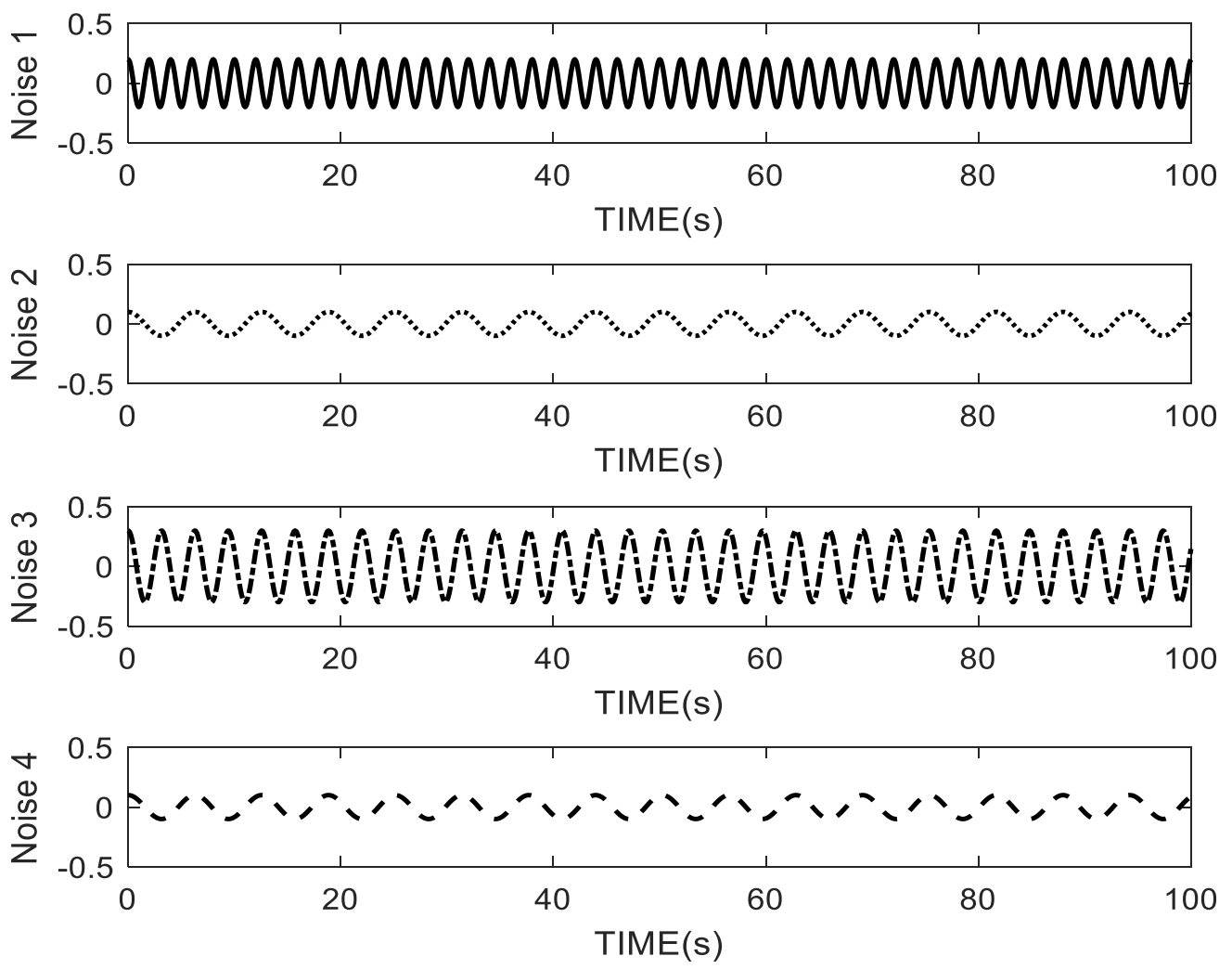

Fig. 18. External noises using for synchronization of the 4D hyperchaotic Rikitake Dynamo System 


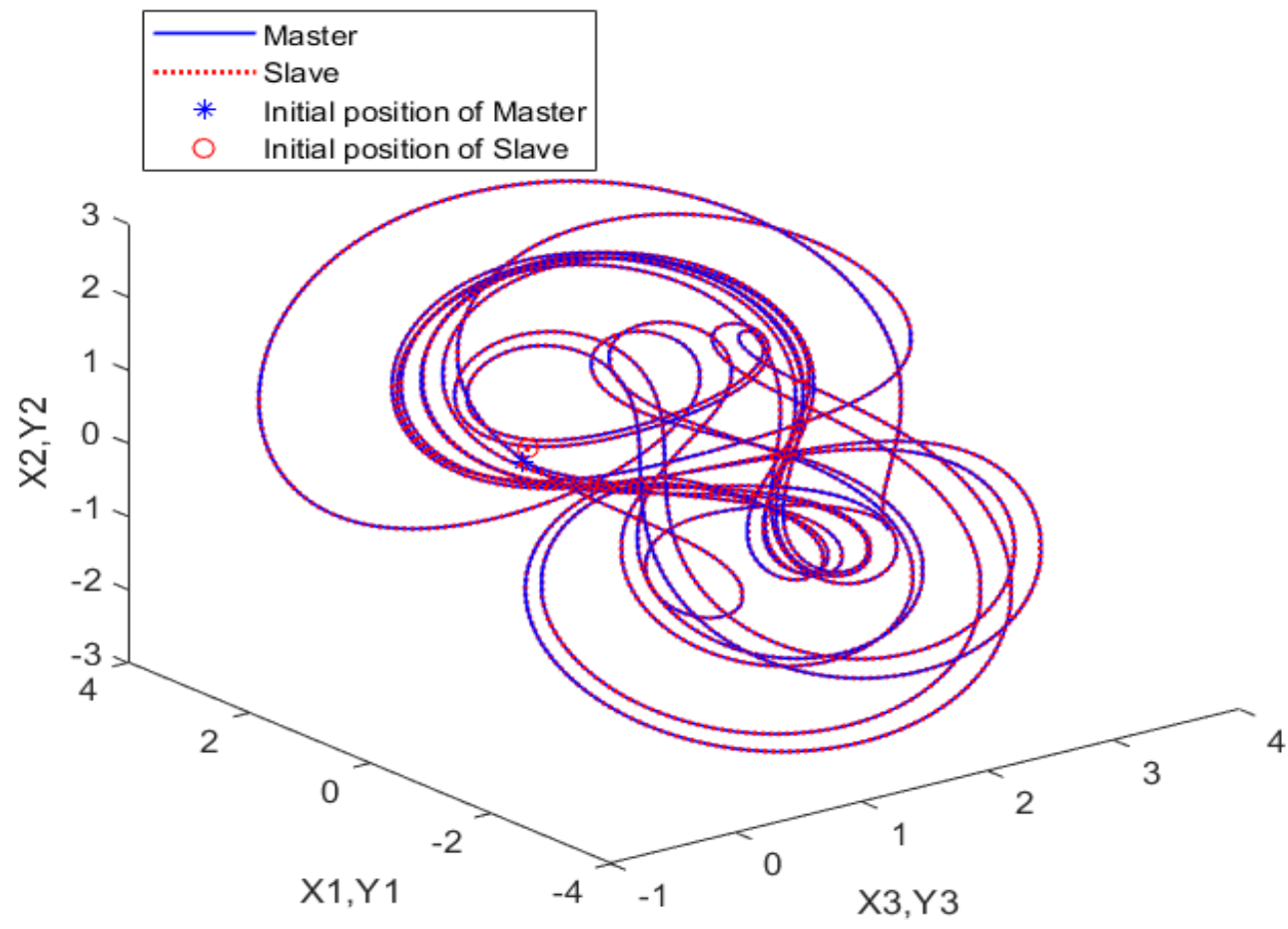

Fig. 19. 3D trajectory phase portrait of master (X1, X2, X3) and slave (Y1, Y2, Y3)

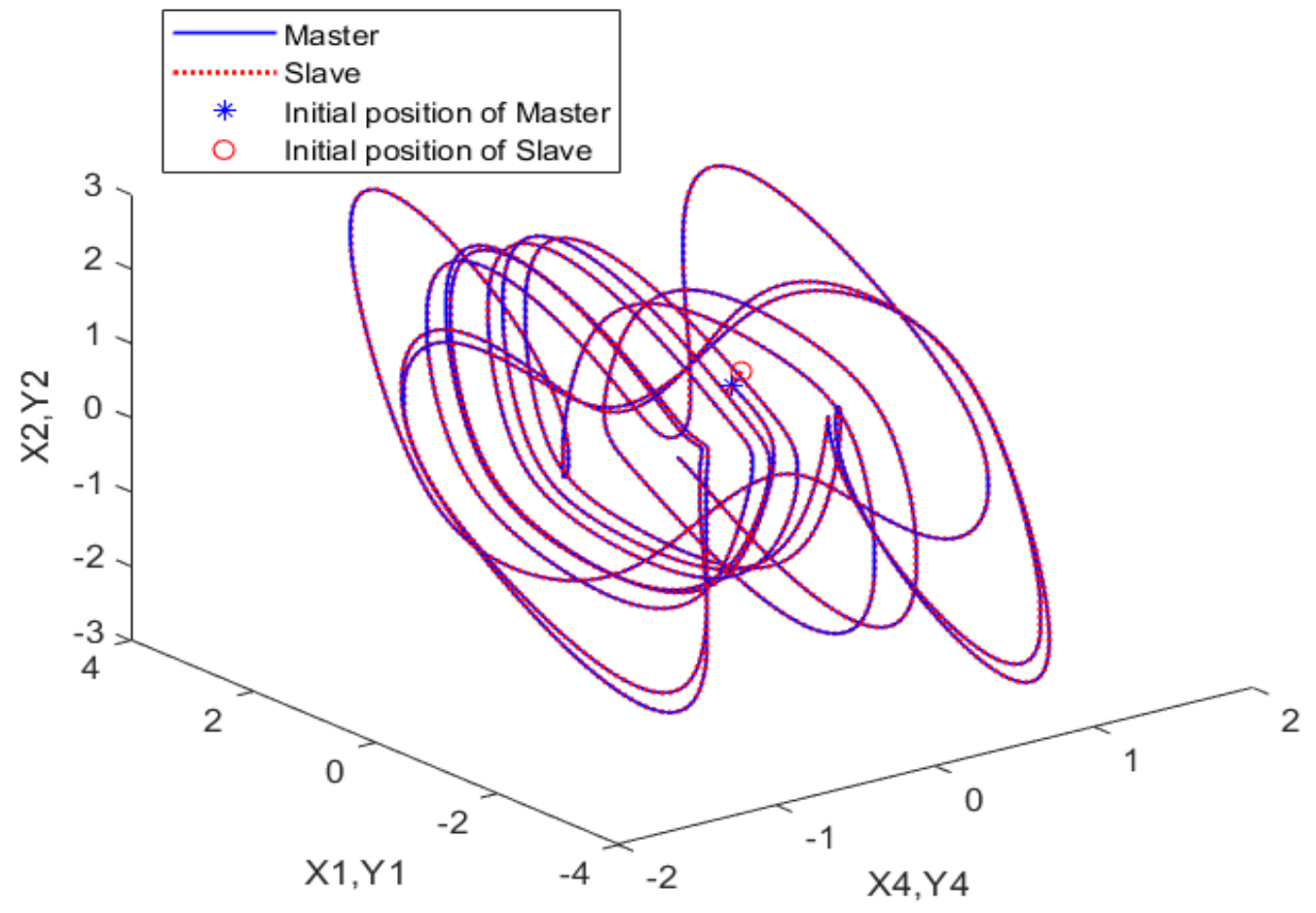

Fig. 20. 3D trajectory phase portrait of master (X1, X2, X4) and slave (Y1, Y2, Y4) 


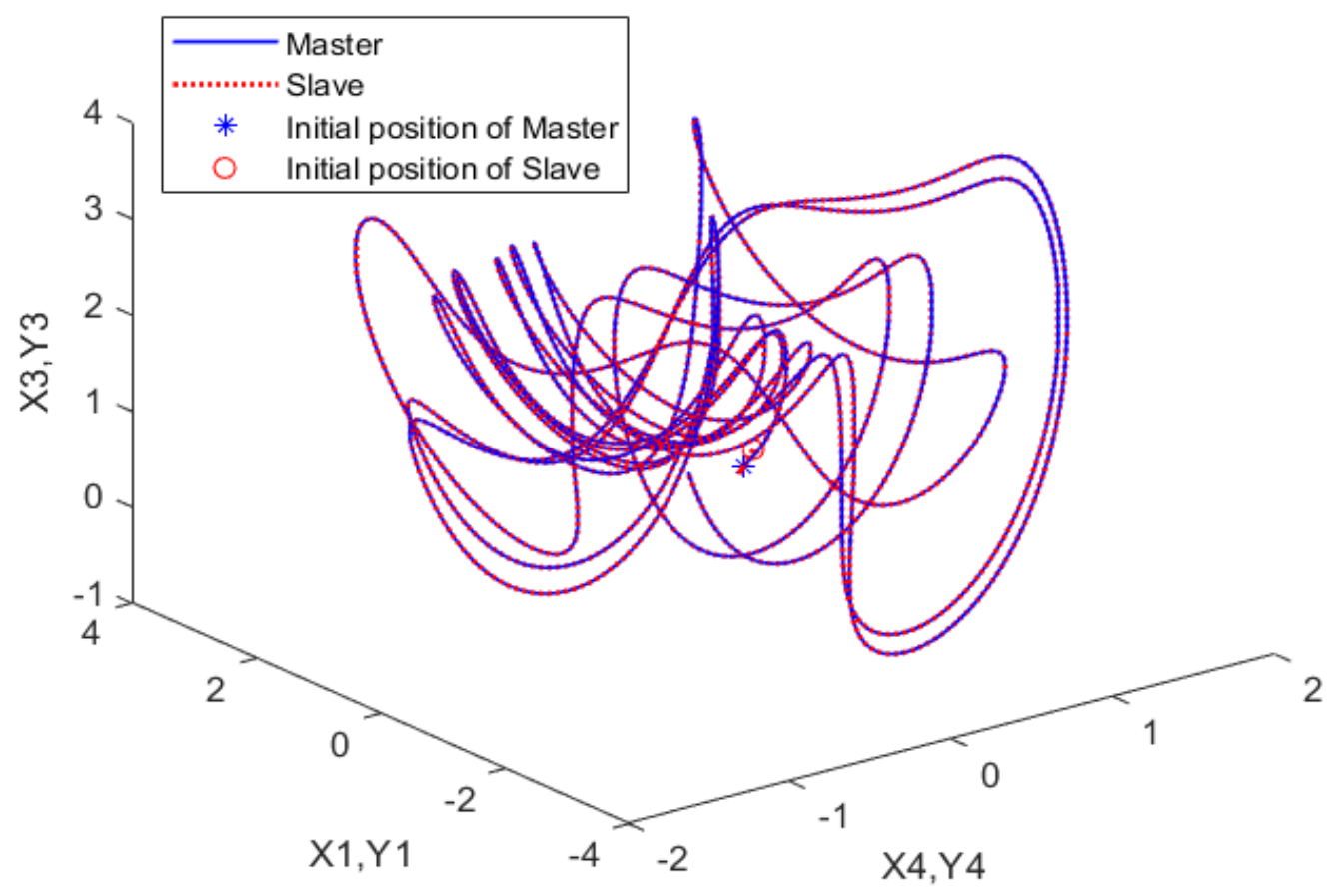

Fig. 21. 3D trajectory phase portrait of master (X1, X3, X4) and slave (Y1, Y3, Y4)

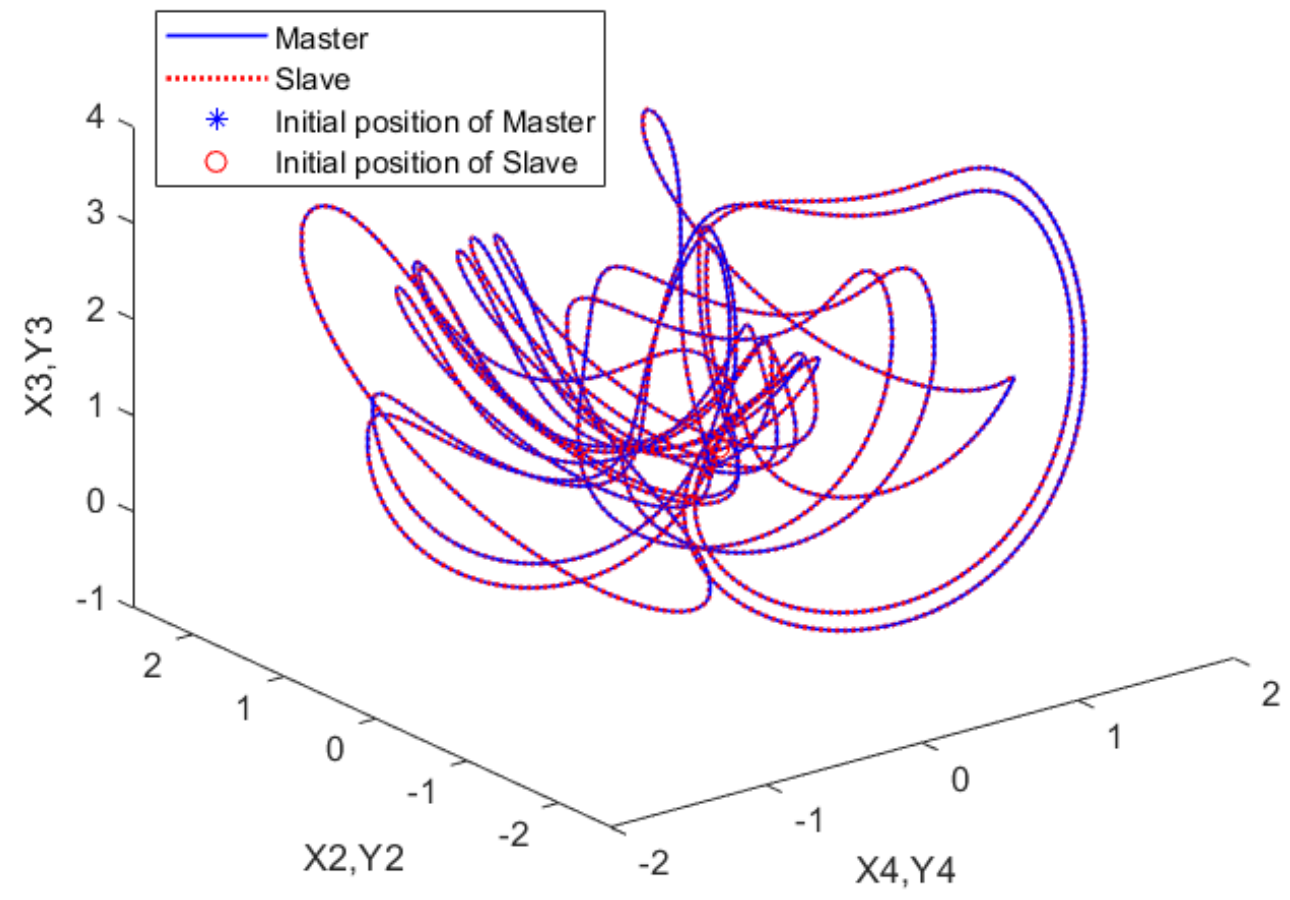

Fig. 22. 3D trajectory phase portrait of master (X2, X3, X4) and slave (Y2, Y3, Y4) 


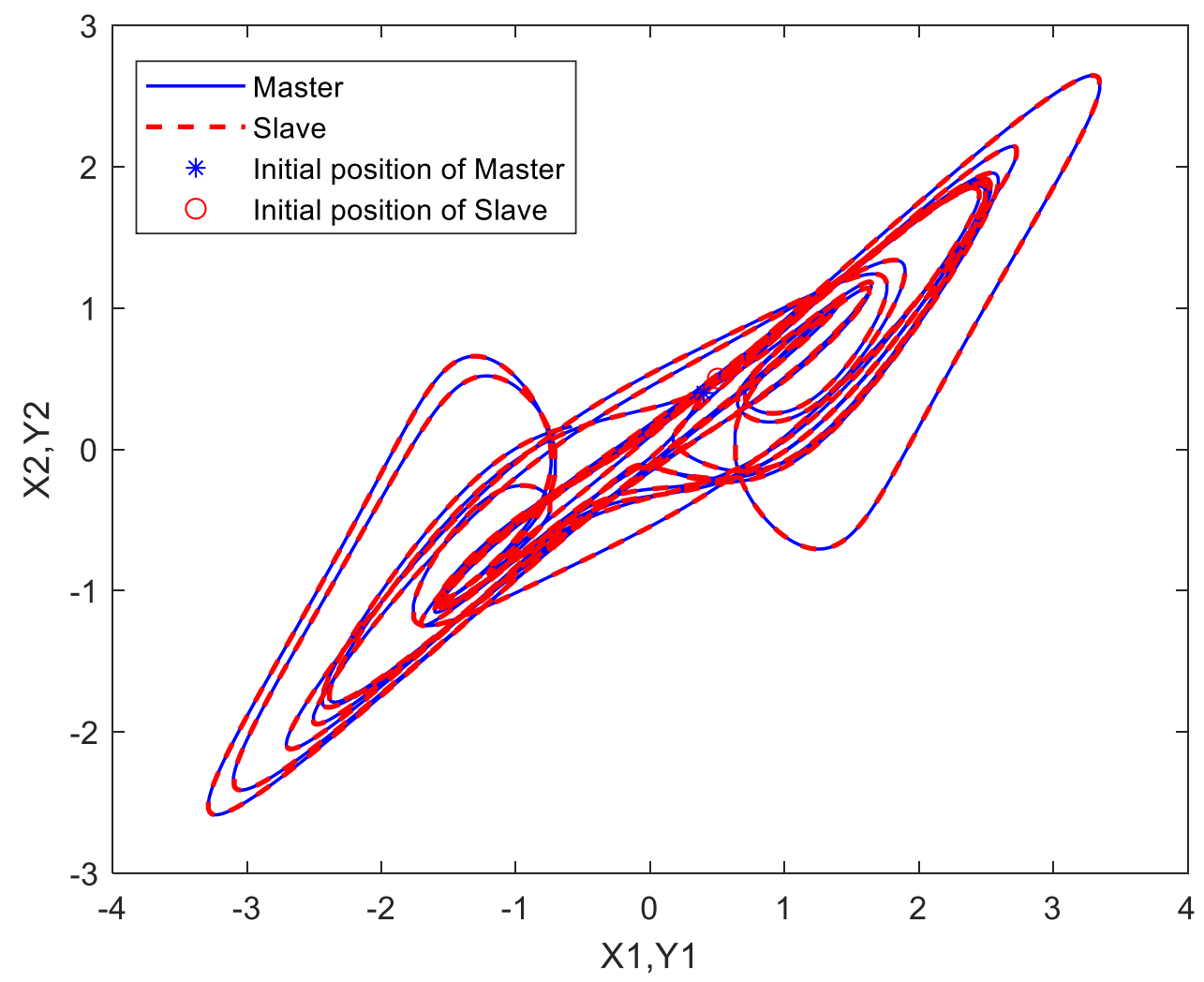

Fig. 23. The state trajectory of master $(\mathrm{X} 1, \mathrm{X} 2)$ and slave $(\mathrm{Y} 1, \mathrm{Y} 2)$

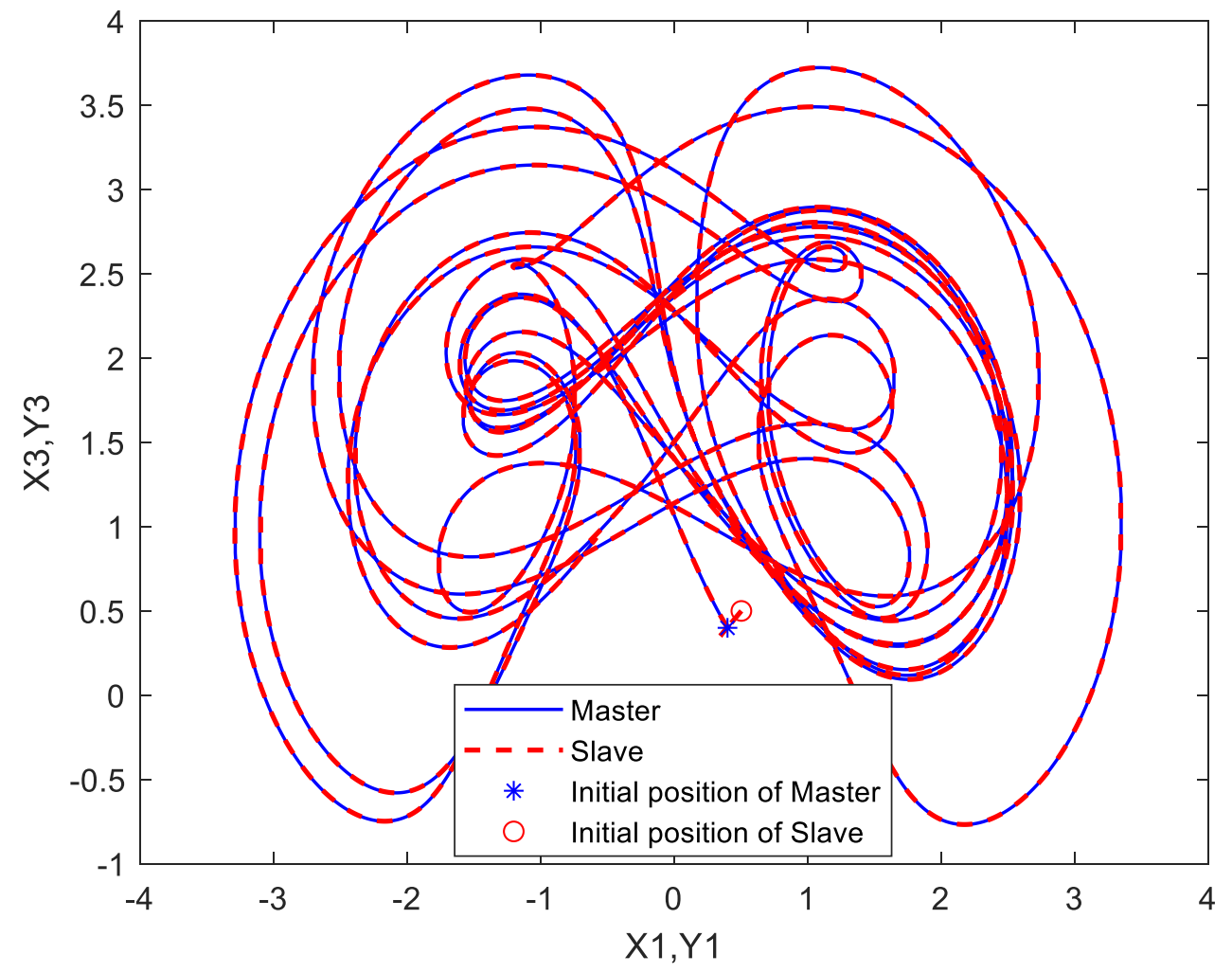

Fig. 24. The state trajectory of master $(\mathrm{X} 1, \mathrm{X} 3)$ and slave $(\mathrm{Y} 1, \mathrm{Y} 3)$ 


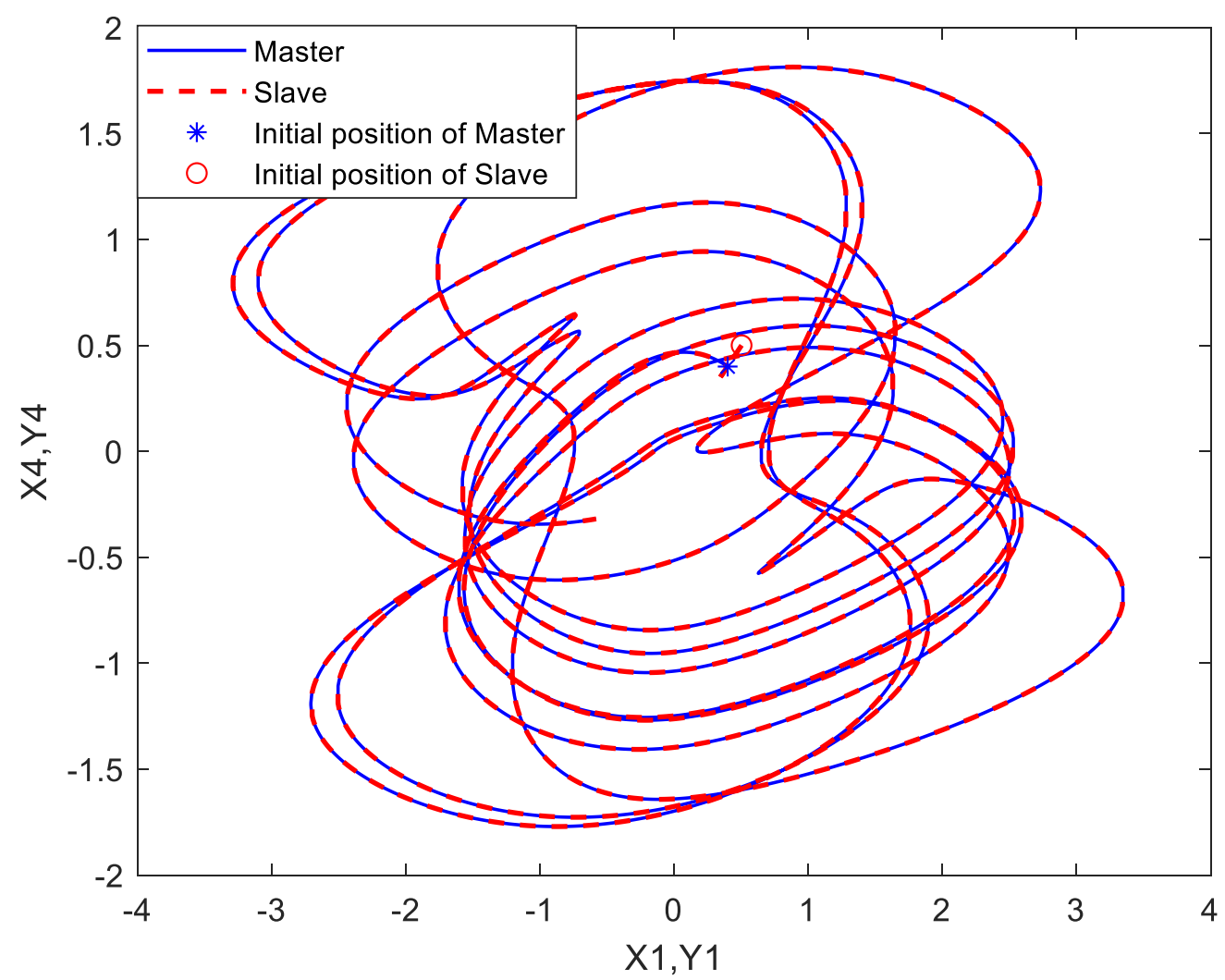

Fig. 25. The state trajectory of master (X1, X4) and slave (Y1, Y4)

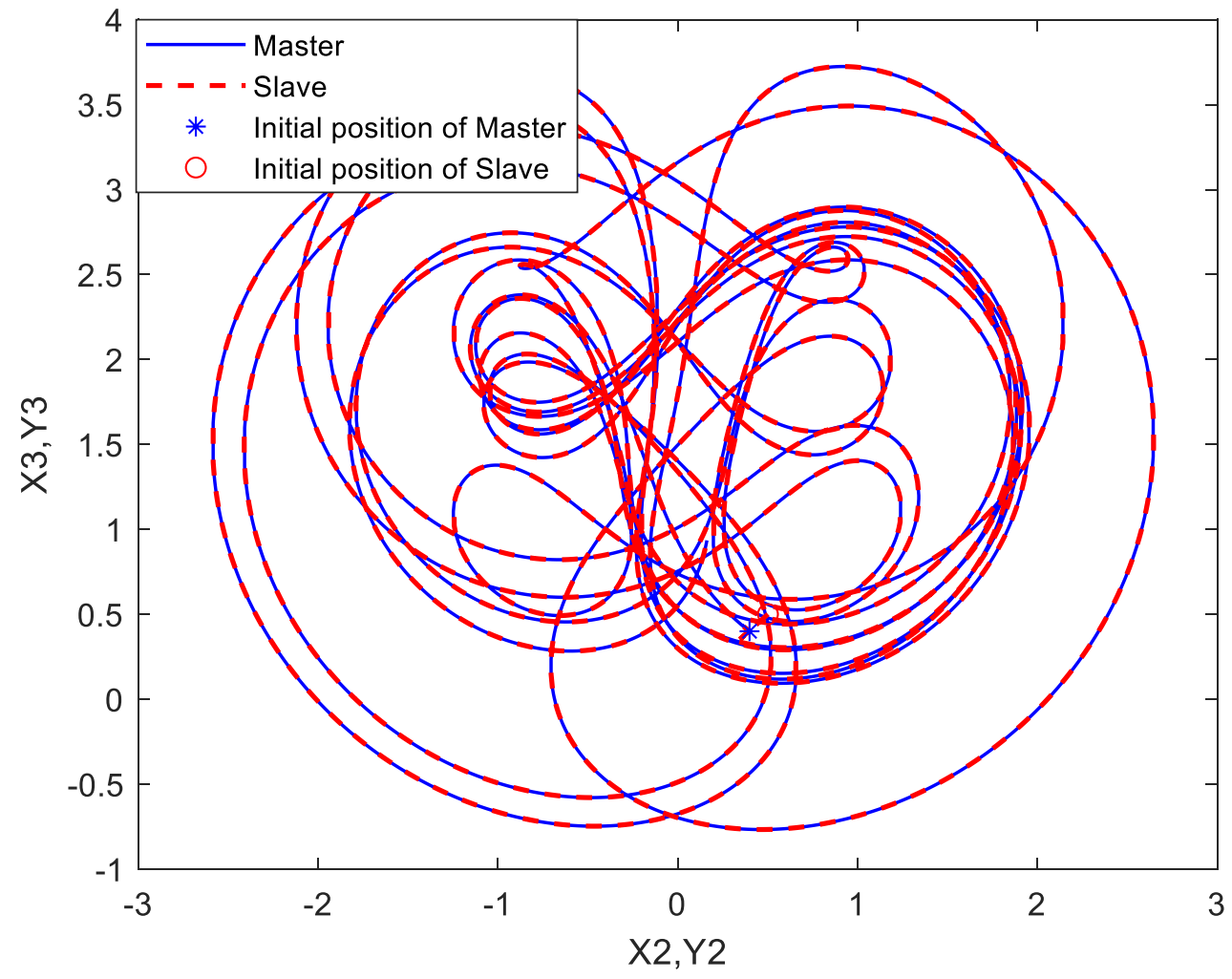

Fig. 26. The state trajectory of master (X2, X3) and slave (Y2, Y3) 


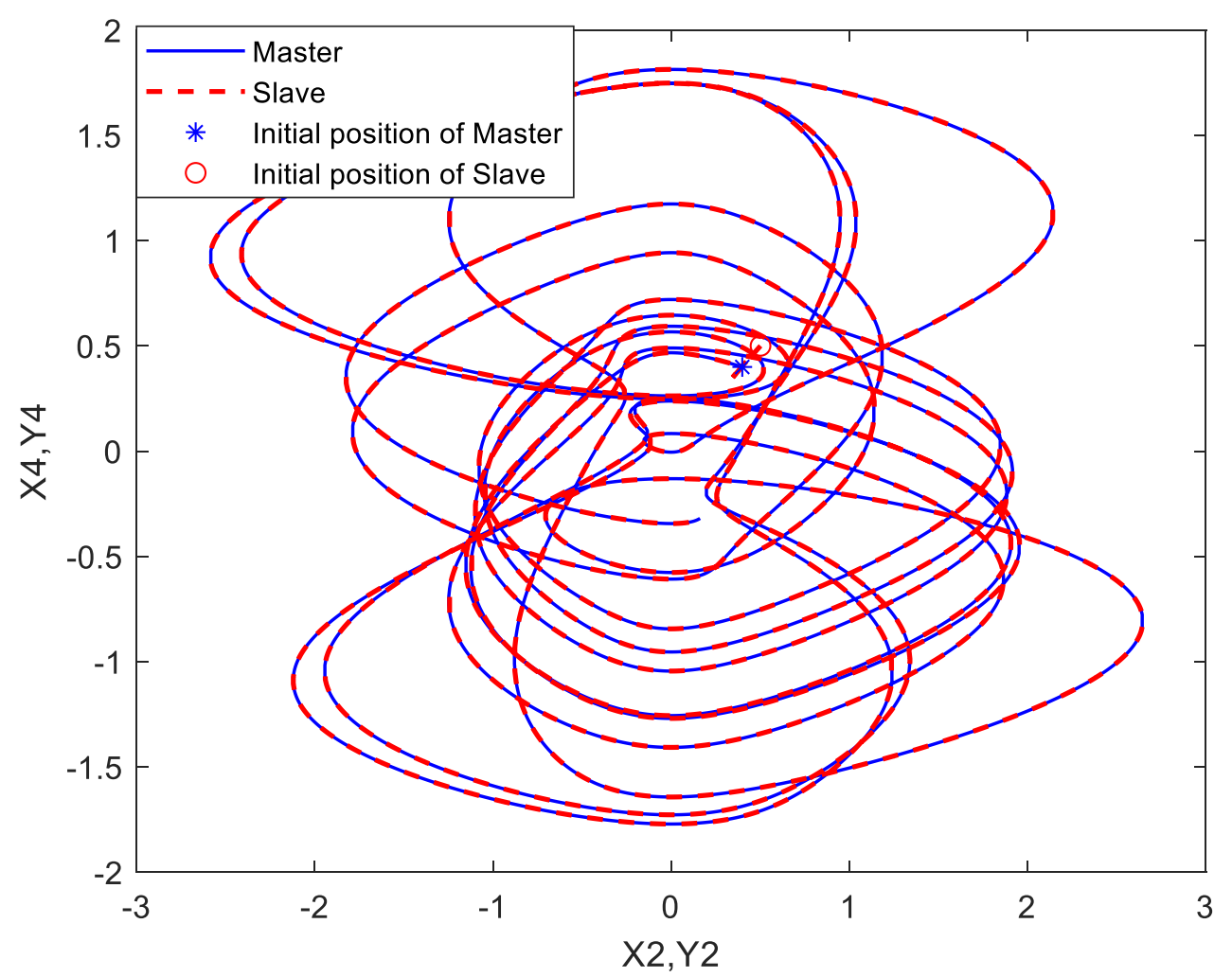

Fig. 27. The state trajectory of master (X2, X4) and slave (Y2, Y4)

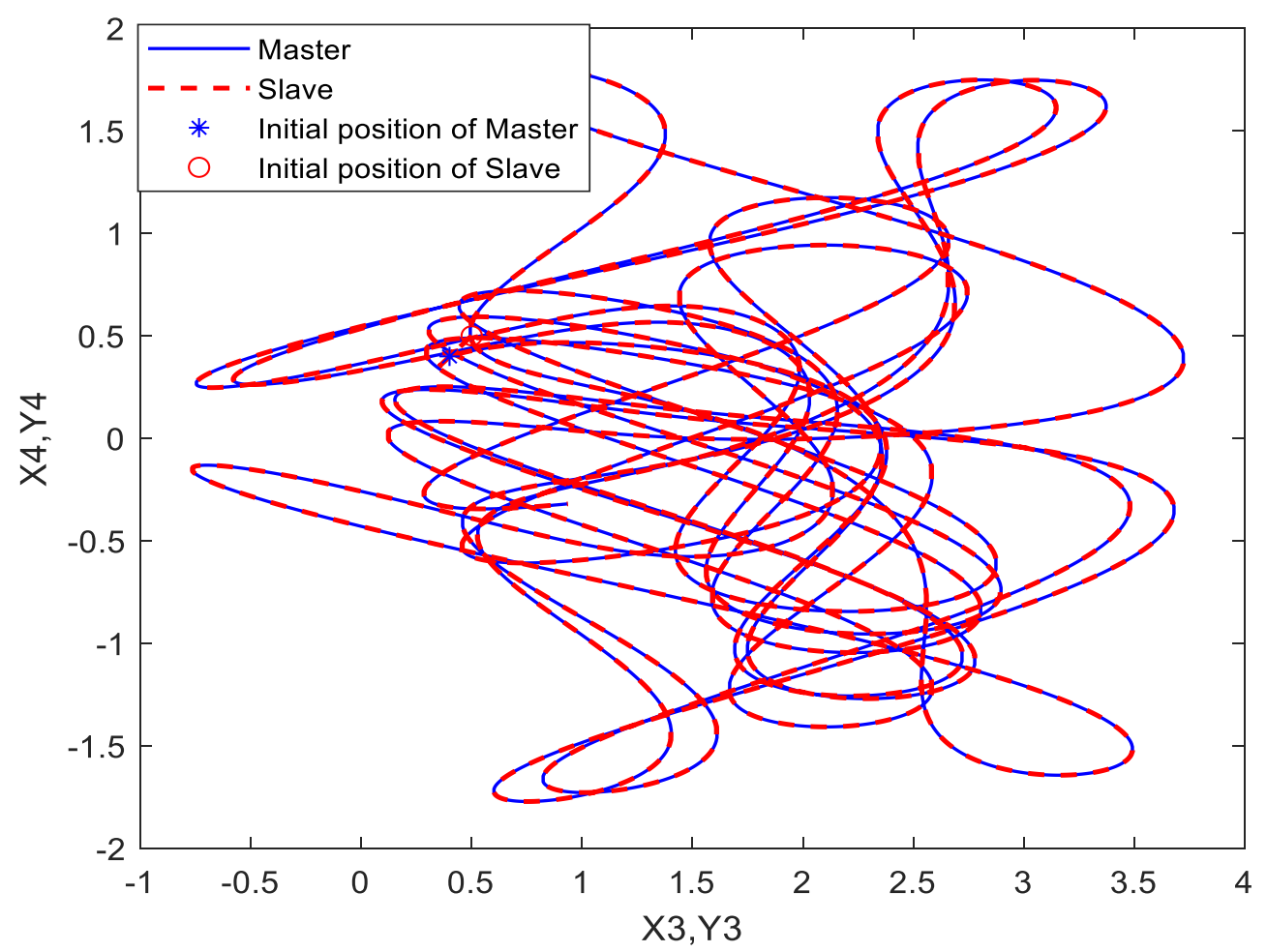

Fig. 28. The state trajectory of master (X3, X4) and slave (Y3, Y4) 

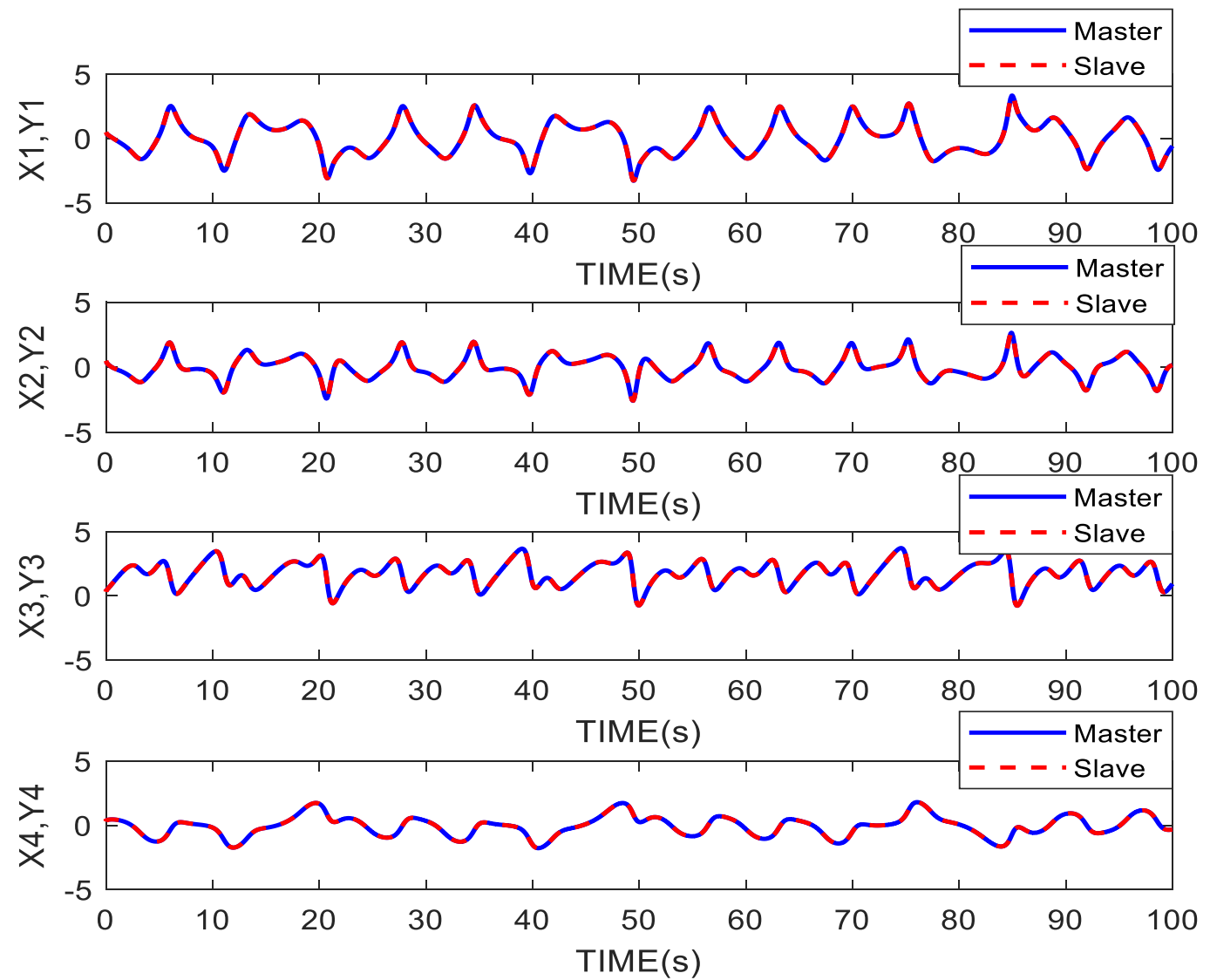

Fig. 29. The state trajectory of master-slave (X1, Y1), (X2, Y2), (X3, Y3), and (X4, Y4)
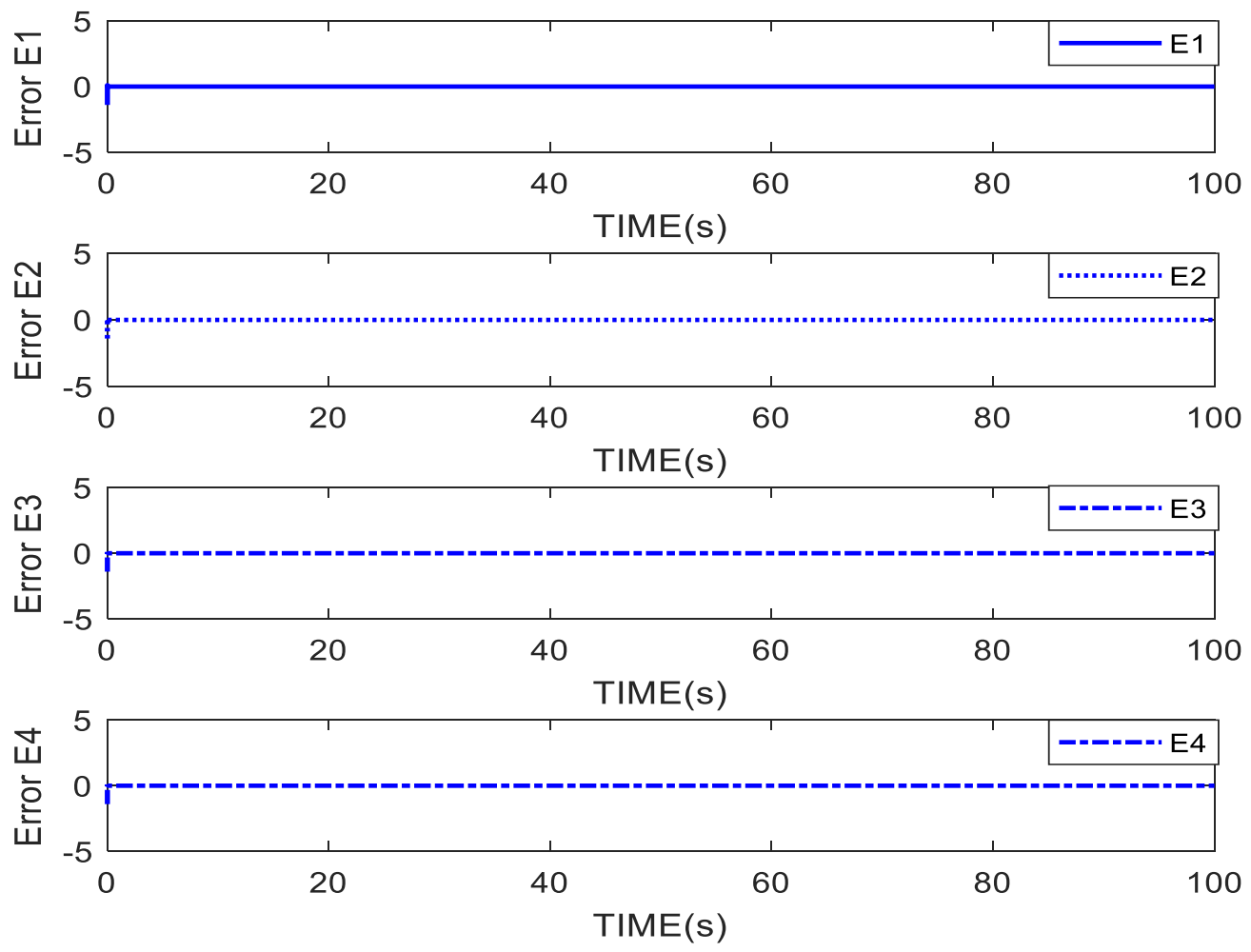

Fig. 30. The errors of synchronization for the 4D hyperchaotic Rikitake Dynamo System 


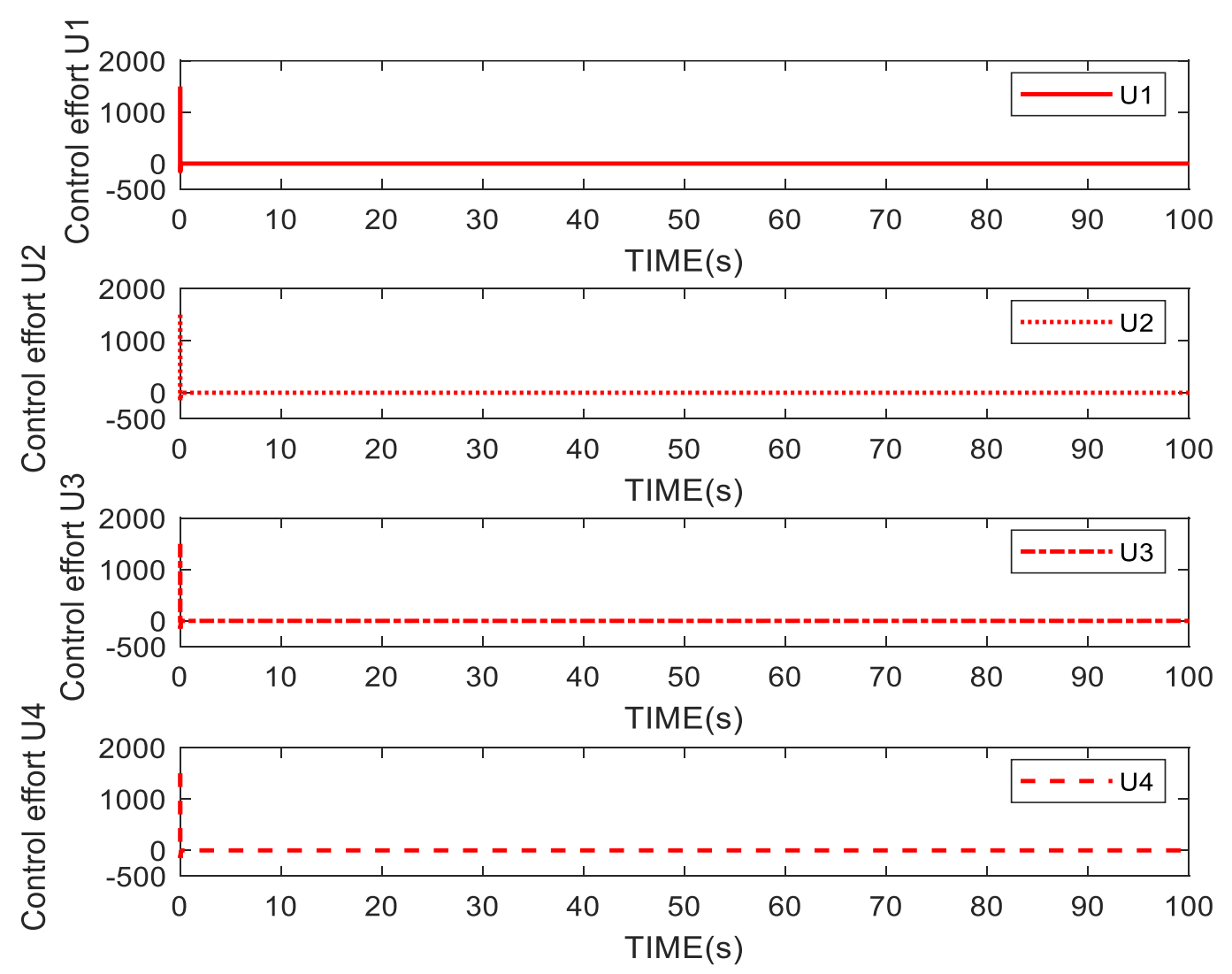

Fig. 31. The control efforts of synchronization for the 4D hyperchaotic Rikitake Dynamo System

Table 2 RMSE for the proposed control system

\begin{tabular}{|l|l|l|l|l|c|}
\hline & RMSE1 & RMSE2 & RMSE3 & RMSE4 & Average RMSE \\
\hline RCMAC & 0.0228 & 0.0195 & 0.0227 & 0.0179 & 0.0207 \\
\hline FBELC & 0.0163 & 0.0141 & 0.0160 & 0.0132 & 0.0149 \\
\hline Brain-imitated neural network & 0.0088 & 0.0078 & 0.0085 & 0.0074 & 0.0081 \\
\hline IT2FFFLBC control system & 0.0040 & 0.0042 & 0.0034 & 0.0044 & 0.0040 \\
\hline
\end{tabular}

\section{Conclusion}

In this research, we design the interval type-2 fuzzy fourfold function-link brain emotional controller for 4D nonlinear hyperchaotic systems. The principal novelty of this research is the successful design of the new fourfold function-link for the IT2FFFLBC that can adjust efficiently the lower and upper weights for orbitofrontal cortex and amygdala networks. A smooth robust compensator is used to eliminate undesired approximate errors and to avoid the chattering phenomenon. Two Lyapunov functions are utilized to determine the online learning laws for tuning network parameters and to prove the stability of the system. Subsequently, simulation results of two 4D hyperchaotic Lorenz-Lu and 4D hyperchaotic Rikitake two-wing dynamo systems show that the IT2FFFLBC control system efficiently 
achieves good synchronization. In summary, the proposed controller can deal with system uncertainty and external noise with small tracking errors. However, the major limitation of the proposed scheme is that the learning rates of the adaptive laws are selected by trial-and-error to improve the control performance. The learning rates are very important and they will change the control performance significantly. Our future work will apply some optimization algorithms to select the optimal learning rates of the proposed control system. The other future study is to apply the proposed control system to some practical nonlinear systems.

\section{Acknowledgments}

The authors appreciate the financial support in part from the Ministry of Science and Technology of Republic of China under grant MOST 109-2811-E-155-504-MY3.

\section{Compliance with ethical standards}

Conflict of Interest The authors declare that they have no conflict of interest.

\section{References}

Adeli H, Jiang X (2008) Intelligent infrastructure: neural networks, wavelets, and chaos theory for intelligent transportation systems and smart structures. CRC press

Andrievsky B, Kudryashova EV, Kuznetsov NV, Kuznetsova OA, Mokaev TN (2018) Hidden nonlinear oscillations in aircraft stabilization system with restrictions at the actuator control. In: AIP Conference Proceedings, 2018. vol 1. AIP Publishing, p 020004

Beal A, Blakely J, Corron N, Dean R (2016) High frequency oscillators for chaotic radar. In: Radar Sensor Technology XX, 2016. International Society for Optics and Photonics, p 98290H

Boubellouta A, Zouari F, Boulkroune A (2019) Intelligent fuzzy controller for chaos synchronization of uncertain fractional-order chaotic systems with input nonlinearities. International Journal of General Systems 48:211-234

Chen A, Lu J, Lü J, Yu S (2006) Generating hyperchaotic Lü attractor via state feedback control. Physica A: Statistical Mechanics and its Applications 364:103-110

Chen C-H, Lin C-M, Li M-C (2011) Development of PI training algorithms for neuro-wavelet control on the synchronization of uncertain chaotic systems Neurocomputing 74:2797-2812

Chen L, Tang S, Li Q, Zhong S (2018) A new 4D hyperchaotic system with high complexity Mathematics and Computers in Simulation 146:44-56

Dashti ZAS, Gholami M, Hajimani M (2017) Brain emotional learning based intelligent controller for velocity control of an electro hydraulic servo system IOSR J Elec Electron Eng 12:29-35

Ding Z, Qiu H, Yang R, Jiang C, Zhou M (2019) Interactive-control-model for Human-computer interactive system based on Petri nets. IEEE Transactions on Automation Science and Engineering 16:1800-1813

Hsu C-F, Chung C-M, Lin C-M, Hsu C-Y (2009) Adaptive CMAC neural control of chaotic systems with a PI-type learning algorithm Expert Systems with Applications 36:11836-11843

Hsu C-F, Su C-T, Lee T-T (2016) Chaos synchronization using brain-emotional-learning-based fuzzy control. In: 2016 Joint 8th International Conference on Soft Computing and Intelligent Systems 
(SCIS) and 17th International Symposium on Advanced Intelligent Systems (ISIS), 2016, pp 811816

Huynh T-T, Le T-L, Lin C-M (2019) Self-organizing recurrent wavelet fuzzy neural network-based control system design for MIMO uncertain nonlinear systems using TOPSIS method. International Journal of Fuzzy Systems 21:468-487

Huynh T-T, Lin C-M, Le T-L, Nguyen NP, Hong S-K, Chao F (2020a) Wavelet interval type-2 fuzzy quad-function-link brain emotional control algorithm for the synchronization of 3D nonlinear chaotic systems. International Journal of Fuzzy Systems 22: 2546-2564

Huynh T-T, Lin C-M, Le T-L, Vu V-P, Chao F (2020b) Self-organizing double function-link fuzzy brain emotional control system design for uncertain nonlinear systems. IEEE Transactions on Systems, Man, and Cybernetics: Systems. https://doi.org/10.1109/TSMC.2020.3036404

Huynh T-T, Lin C-M, Pham T-TT, Cho H-Y, Le T-L (2019) A modified function-link fuzzy cerebellar model articulation controller using a PI-type learning algorithm for nonlinear system synchronization and control Chaos, Solitons \& Fractals 118:65-82

Huynh TT, Lin CM (2019) Wavelet dual function-link fuzzy brain emotional learning system design for system identification and trajectory tracking of nonlinear systems. In: 2019 IEEE International Conference on Systems, Man and Cybernetics (SMC), 2019, pp 1653-1657

Huynh TT, Lin CM, Le TL (2020) A double function-link function-based fuzzy brain emotional controller for synchronizing a 4D hyper-chaotic system. In: 2020 IEEE International Conference on Systems, Man, and Cybernetics (SMC), 2020, pp 1961-1965

Huynh T-T, Le T-L, Lin C-M (2020) A TOPSIS multi-criteria decision method-based intelligent recurrent wavelet CMAC control system design for MIMO uncertain nonlinear systems. Neural Computing and Applications 32: 4025-4043

Kong Y, Gao J, Xu Y, Pan Y, Wang J, Liu J (2019) Classification of autism spectrum disorder by combining brain connectivity and deep neural network classifier Neurocomputing 324:63-68

Le T-L (2019) Fuzzy C-means clustering interval type-2 cerebellar model articulation neural network for medical data classification. IEEE Access 7:20967-20973

Le T-L, Huynh T-T, Hong S-K (2020a) Self-organizing interval type-2 fuzzy asymmetric CMAC design to synchronize chaotic satellite systems using a modified grey wolf optimizer. IEEE Access 8:5369753709

Le T-L, Lin C-M, Huynh T-T (2018) Self-evolving type-2 fuzzy brain emotional learning control design for chaotic systems using PSO. Applied Soft Computing 73:418-433

Le T-L, Huynh T-T, Hong SK (2020b) A modified grey wolf optimizer for optimum parameters of multilayer type-2 asymmetric fuzzy controller. IEEE Access 8:121611-121629

LeDoux J (1991) Emotion and the limbic system concept Concepts in neuroscience 2:169-199

Lin C-M, Chung C-C (2015) Fuzzy brain emotional learning control system design for nonlinear systems. International Journal of Fuzzy Systems 17:117-128

Lin C-M, Huynh T-T (2018) Function-link fuzzy cerebellar model articulation controller design for nonlinear chaotic systems using TOPSIS multiple attribute decision-making method. International Journal of Fuzzy Systems 20:1839-1856

Lin C-M, Huynh T-T (2019) Dynamic TOPSIS fuzzy cerebellar model articulation controller for magnetic levitation system. Journal of Intelligent \& Fuzzy Systems 36:2465-2480

Lin C-M, Huynh T-T, Le T-L (2018) Adaptive TOPSIS fuzzy CMAC back-stepping control system design for nonlinear systems. Soft Computing 23: 6947-6966

Lin C-M, Nguyen HB, Huynh T-T (2021) A new self-organizing double function-link brain emotional learning controller for MIMO nonlinear systems using sliding surface. IEEE Access 9: 73826-73842

Lin C-M, Pham DH, Huynh T-T (2021) Synchronization of chaotic system using a brain-imitated neural network controller and its applications for secure communications. IEEE Access 9: 75923-75944 
Marzbanrad J, Babalooei M (2016) Grazing Bifurcations and Chaos of a Hydraulic Engine Mount International Journal of Automotive Engineering 6:2182-2190

Mendel JM, Chimatapu R, Hagras H (2020) Comparing the performance potentials of singleton and nonsingleton type-1 and interval type-2 fuzzy systems in terms of sculpting the state space. IEEE Transactions on Fuzzy Systems 28: 783-794

Mendel JM (2011) Uncertain rule-based fuzzy logic systems: introduction and new directions. Prentice Hall, Upper Saddle River

Ni P, Xia Y, Li J, Hao H (2019) Using polynomial chaos expansion for uncertainty and sensitivity analysis of bridge structures. Mechanical Systems and Signal Processing 119:293-311

Panahi S, Pham V-T, Rajagopal K, Boubaker O, Jafari S (2019) A new four-dimensional chaotic system with no equilibrium point. in: recent advances in chaotic systems and synchronization. Elsevier, pp 63-76

Patra JC, Pal RN (1995) A functional link artificial neural network for adaptive channel equalization. Signal Processing 43:181-195

Pham V-T, Kingni ST, Volos C, Jafari S, Kapitaniak T (2017) A simple three-dimensional fractionalorder chaotic system without equilibrium: Dynamics, circuitry implementation, chaos control and synchronization. AEU-International Journal of Electronics and Communications 78:220-227

Rahmani M, Ghanbari A, Ettefagh MM (2018) A novel adaptive neural network integral sliding-mode control of a biped robot using bat algorithm. Journal of Vibration and Control 24:2045-2060

Rakheja P, Vig R, Singh P (2019) Optical asymmetric watermarking using 4D hyperchaotic system and modified equal modulus decomposition in hybrid multi resolution wavelet domain. Optik 176:425437

Ravi V, Pradeepkumar D, Deb K (2017) Financial time series prediction using hybrids of chaos theory, multi-layer perceptron and multi-objective evolutionary algorithms. Swarm and Evolutionary Computation 36:136-149

Rong N, Wang Z, Ding S, Zhang H (2018) Interval type-2 regional switching T-S fuzzy control for timedelay systems via membership function dependent approach. Fuzzy Sets and Systems 374:152-169

Sambas A, He S, Liu H, Vaidyanathan S, Hidayat Y, Saputra J (2020) Dynamical analysis and adaptive fuzzy control for the fractional-order financial risk chaotic system. Advances in Difference Equations 2020:1-12

Sambas A, Mamat M, Arafa AA, Mahmoud GM, Mohamed MA, Sanjaya W (2019a) A new chaotic system with line of equilibria: dynamics, passive control and circuit design. International Journal of Electrical \& Computer Engineering 9:2088-8708

Sambas A, Mamat M, Viadyanathan S, Mohamed MA, Sanjaya WM (2018) A new 4-D chaotic system with hidden attractor and its circuit implementation. International Journal of Engineering \& Technology 7:1245-1250

Sambas A, Vaidyanathan S, Zhang S, Mohamed MA, Zeng Y, Azar AT (2021) A new 4-D chaotic hyperjerk system with coexisting attractors, its active backstepping control, and circuit realization. In: Backstepping Control of Nonlinear Dynamical Systems. Elsevier, pp 73-94

Sambas A, Vaidyanathan S, Zhang S, Putra WT, Mamat M, Mohamed MA (2019b) Multistability in a novel chaotic system with perpendicular lines of equilibrium: analysis, adaptive synchronization and circuit design. Engineering Letters 27

Slotine JJE, Li W (1991) Applied Nonlinear Control. Prentice-Hall, Englewood Cliffs

Sothmann B, Sánchez R, Jordan AN, Büttiker M (2012) Rectification of thermal fluctuations in a chaotic cavity heat engine Physical Review B 85:205301

Sun J, Shen Y, Yin Q, Xu C (2013) Compound synchronization of four memristor chaotic oscillator systems and secure communication Chaos: An Interdisciplinary. Journal of Nonlinear Science 23:013140 
Vaidyanathan S, Dolvis LG, Jacques K, Lien C-H, Sambas A (2019) A new five-dimensional four-wing hyperchaotic system with hidden attractor, its electronic circuit realisation and synchronisation via integral sliding mode control. International Journal of Modelling, Identification and Control 32:3045

Vaidyanathan S, Pham V-T, Volos C, Sambas A (2018) A novel 4-D hyperchaotic Rikitake Dynamo system with hidden attractor, its properties, synchronization and circuit design. In: Pham V-T, Vaidyanathan S, Volos C, Kapitaniak T (eds) Nonlinear Dynamical Systems with Self-Excited and Hidden Attractors. Springer International Publishing, Cham, pp 345-364

Vaidyanathan S, Rasappan S (2014) Global chaos synchronization of n-scroll chua circuit and lur'e system using backstepping control design with recursive feedback. Arabian Journal for Science \& Engineering (Springer Science \& Business Media BV) 39

Wang H, Luo C, Wang X (2019a) Synchronization and identification of nonlinear systems by using a novel self-evolving interval type-2 fuzzy LSTM-neural network. Engineering Applications of Artificial Intelligence 81:79-93

Wang X, Kingni ST, Volos C, Pham VT, Vo HD, Jafari S (2019b) A fractional system with five terms: analysis, circuit, chaos control and synchronization. International Journal of Electronics 106:109-120

Wang Y, Shen H, Karimi HR, Duan D (2018) Dissipativity-based fuzzy integral sliding mode control of continuous-time TS fuzzy systems. IEEE Transactions on Fuzzy Systems 26:1164-1176

Wu J, Liao X, Yang B (2017) Color image encryption based on chaotic systems and elliptic curve ElGamal scheme. Signal Processing 141:109-124

Xue W, Zhang M, Liu S, Li Y, Cang S (2019) Mechanical analysis and ultimate boundary estimation of the chaotic permanent magnet synchronous motor. Journal of the Franklin Institute 356: 5378-5394

Zhao J, Lin C-M (2019) Wavelet-TSK-type fuzzy cerebellar model neural network for uncertain nonlinear systems. IEEE Transactions on Fuzzy Systems 27:549-558

Zhou Q, Chao F, Lin C-M (2018) A functional-link-based fuzzy brain emotional learning network for breast tumor classification and chaotic system synchronization. International Journal of Fuzzy Systems 20:349-365 
Figures

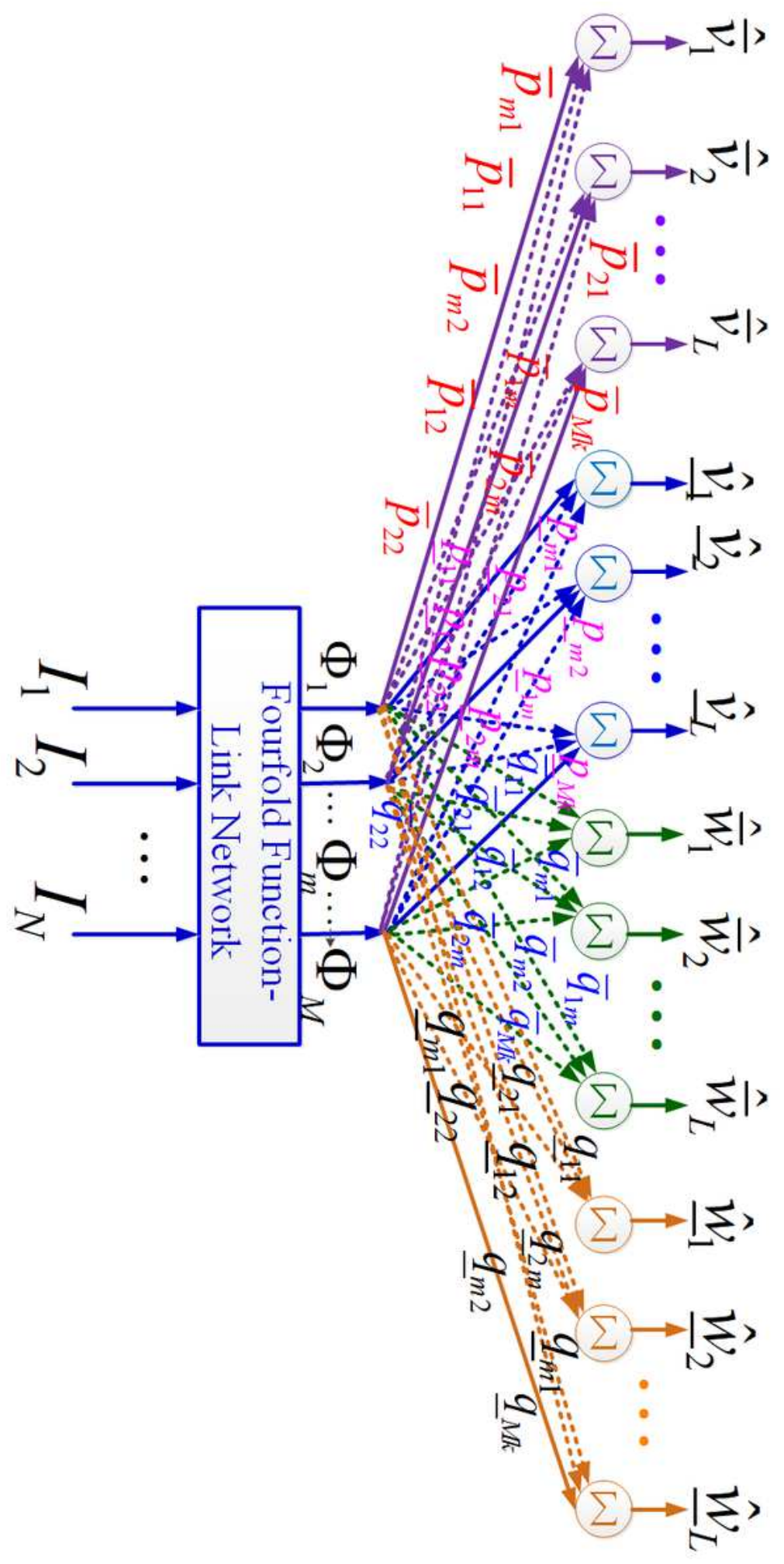

Figure 1

Fourfold FLN 


\section{Layer 1 Layer 2 Layer $3 \quad$ Layer $4 \quad$ Layer 5}
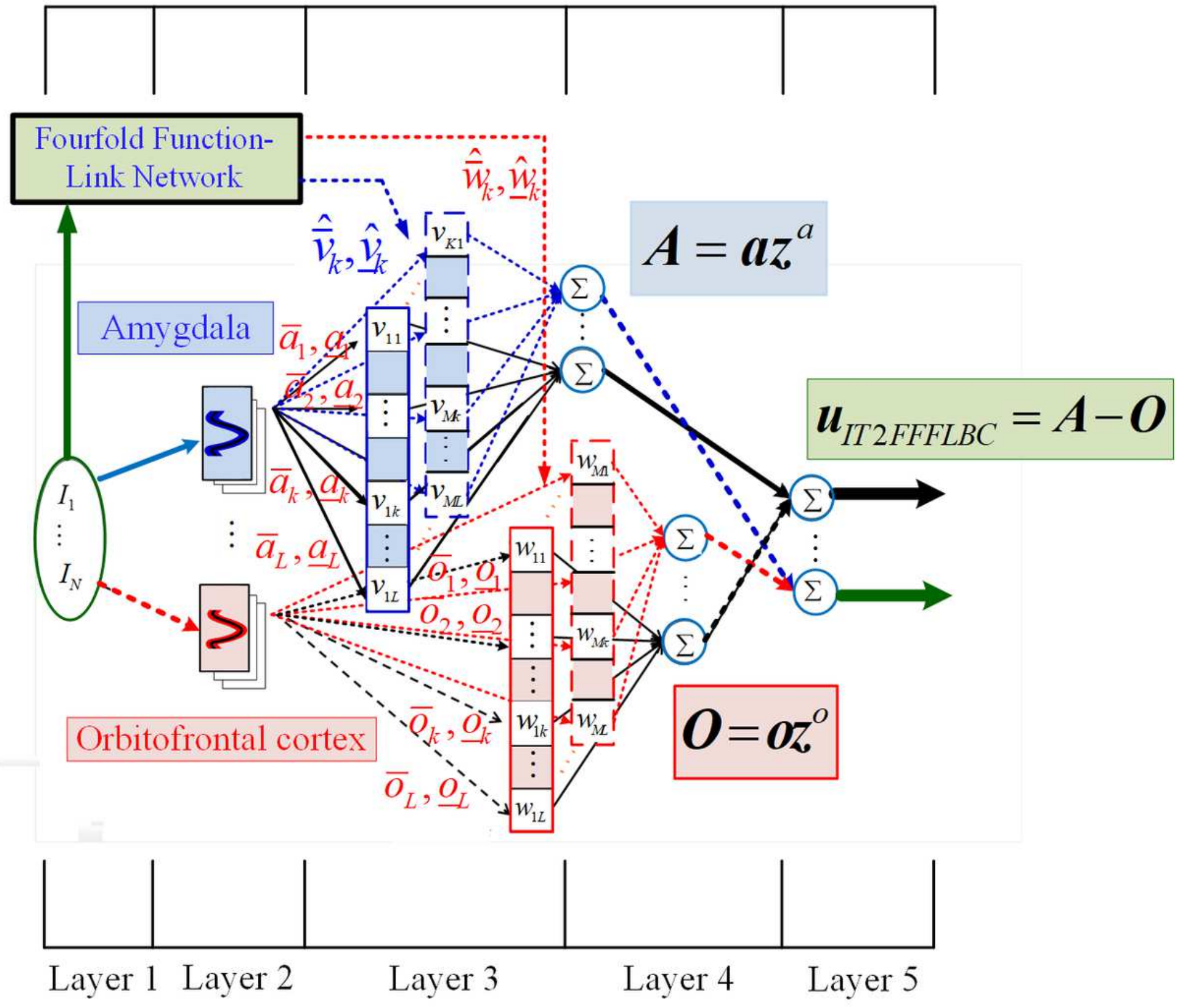

Figure 2

Structure of IT2FFFLBC 


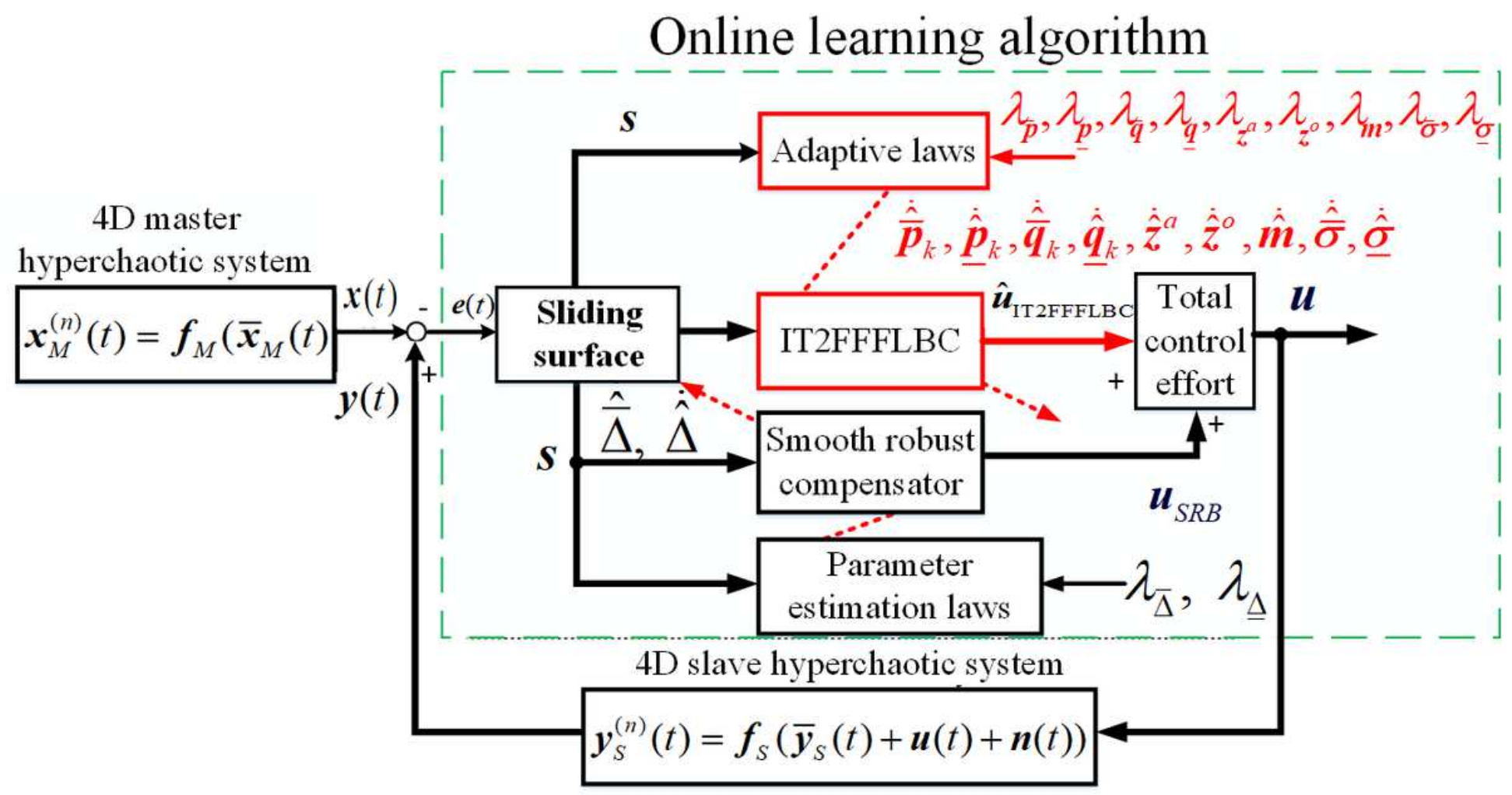

Figure 3

Block diagram of synchronization for 4D nonlinear hyperchaotic systems using the proposed IT2FFFLBC 

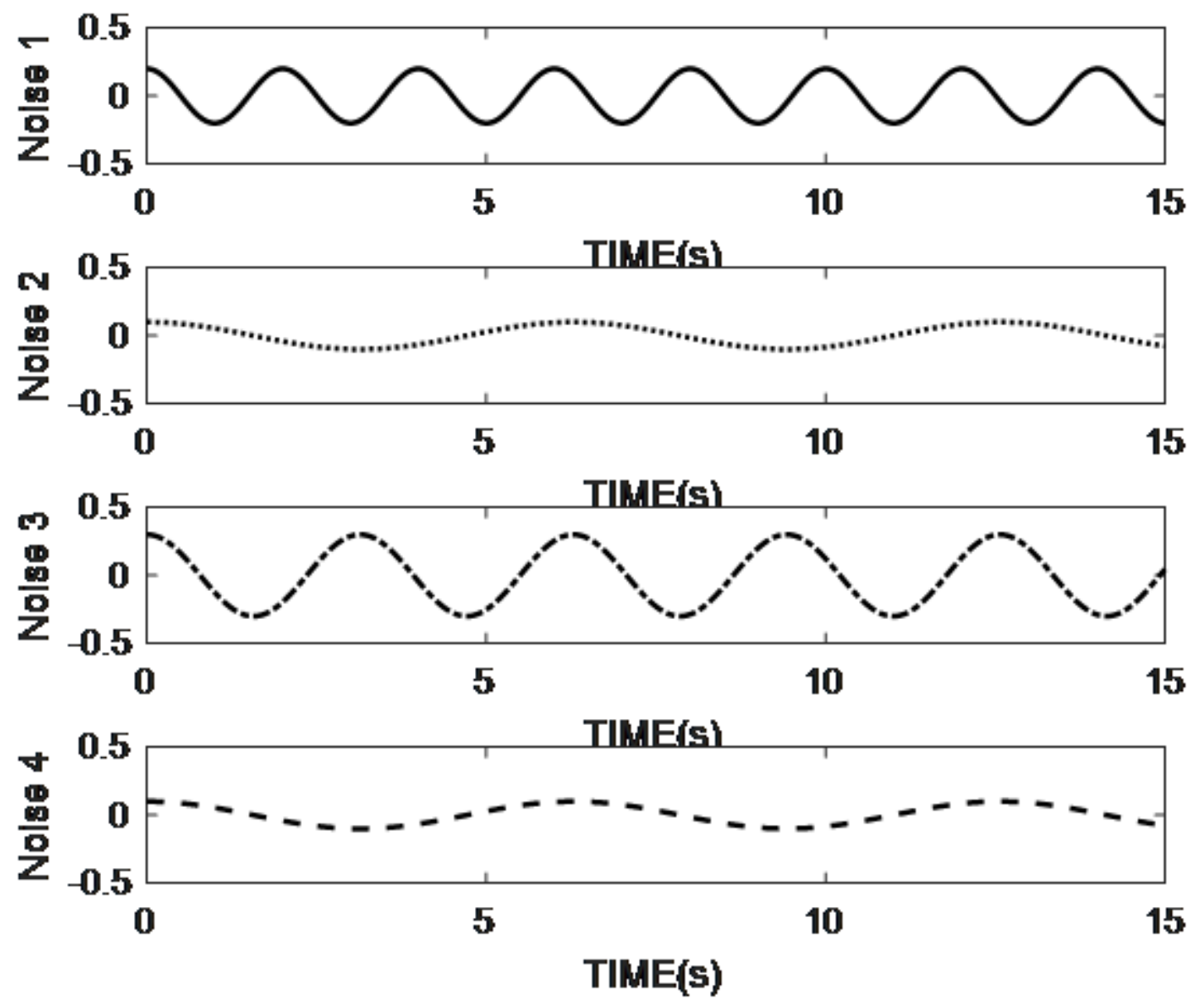

Figure 4

External noises using for synchronization of the 4D hyperchaotic Lorenz-Lu system 


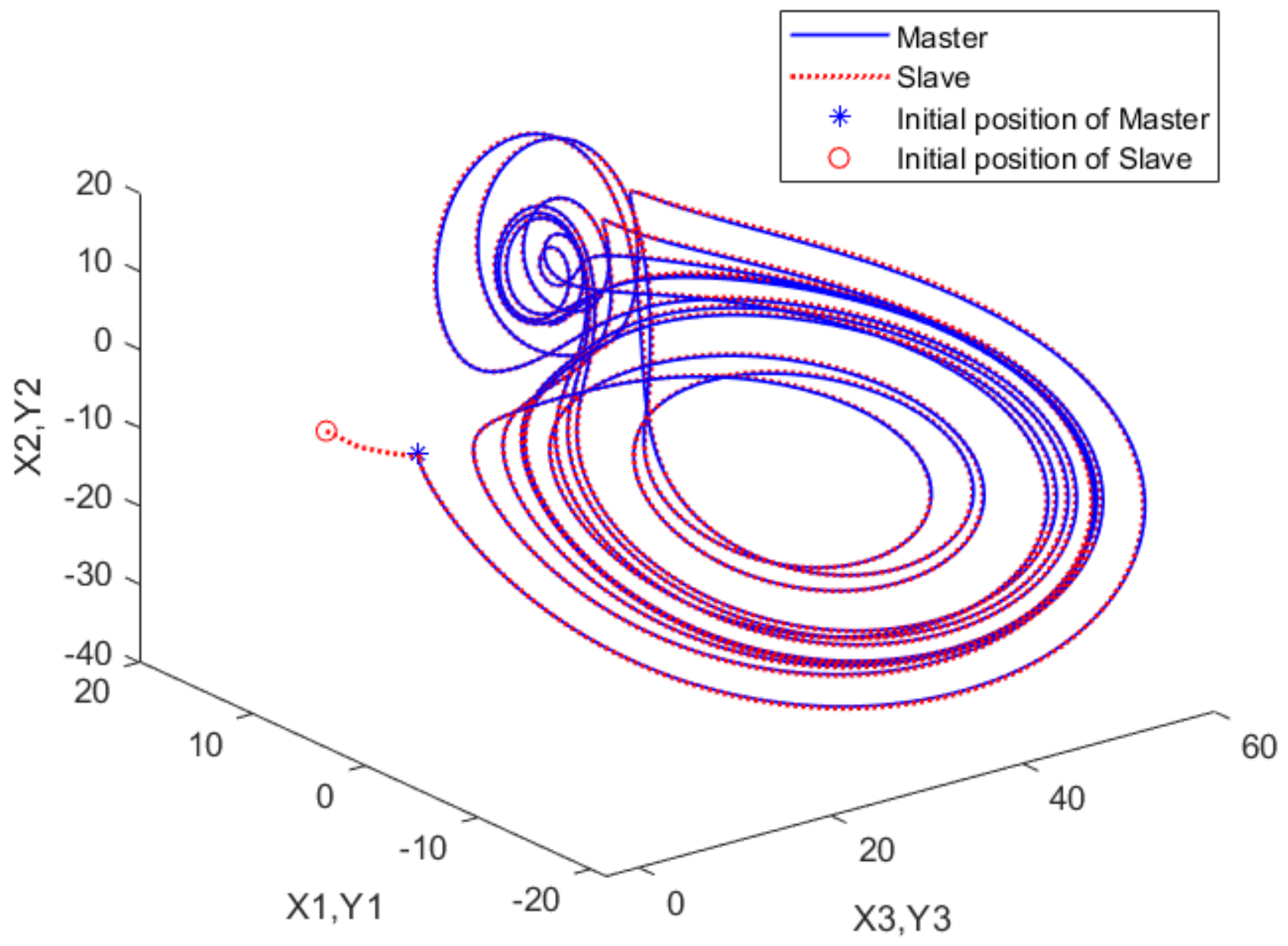

Figure 5

3D trajectory phase portrait of master $(\mathrm{X} 1, \mathrm{X} 2, \mathrm{X} 3)$ and slave $(\mathrm{Y} 1, \mathrm{Y} 2, \mathrm{Y} 3)$ 


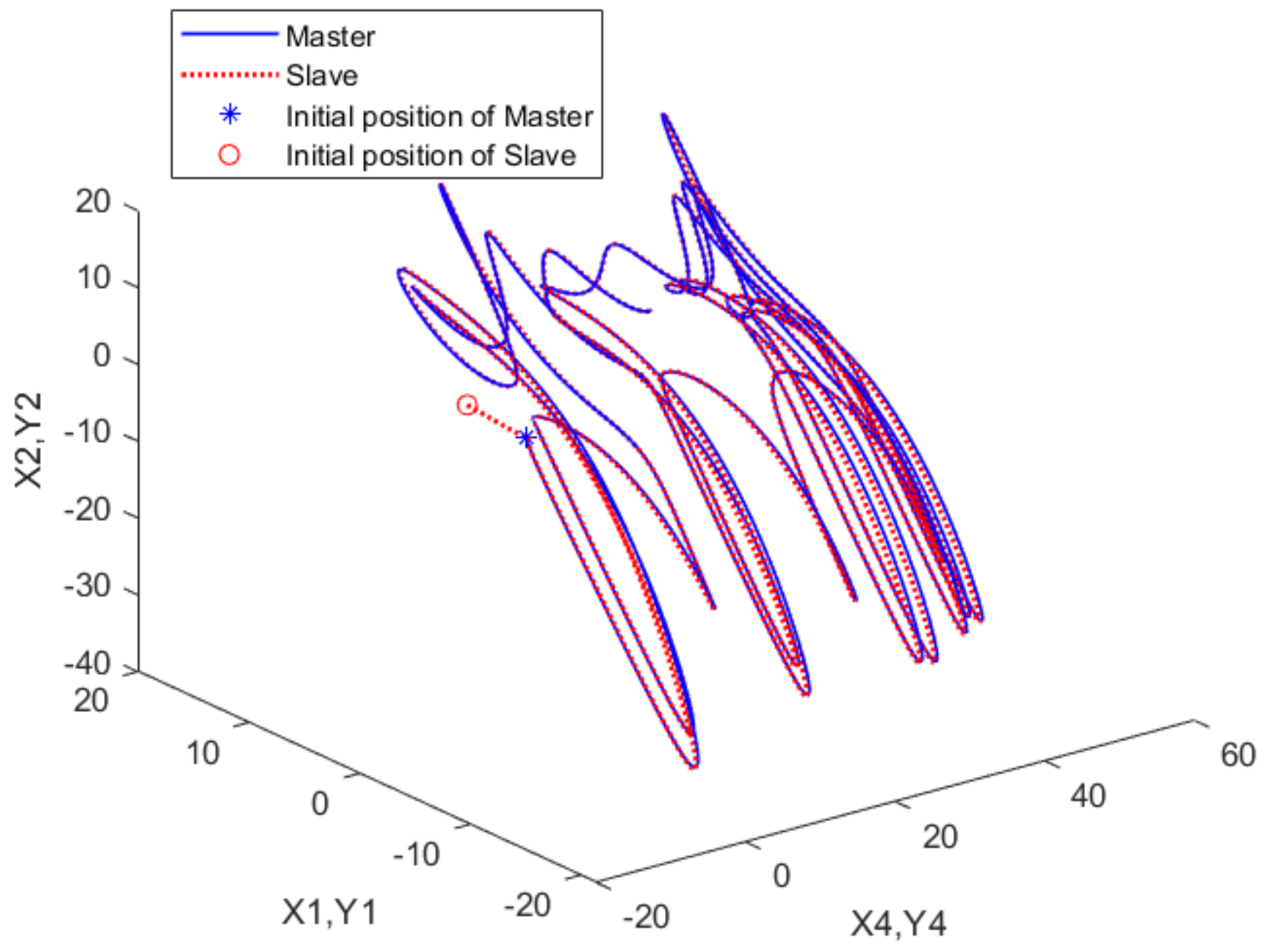

Figure 6

3D trajectory phase portrait of master $(\mathrm{X} 1, \mathrm{X} 2, \mathrm{X} 4)$ and slave $(\mathrm{Y} 1, \mathrm{Y} 2, \mathrm{Y} 4)$ 


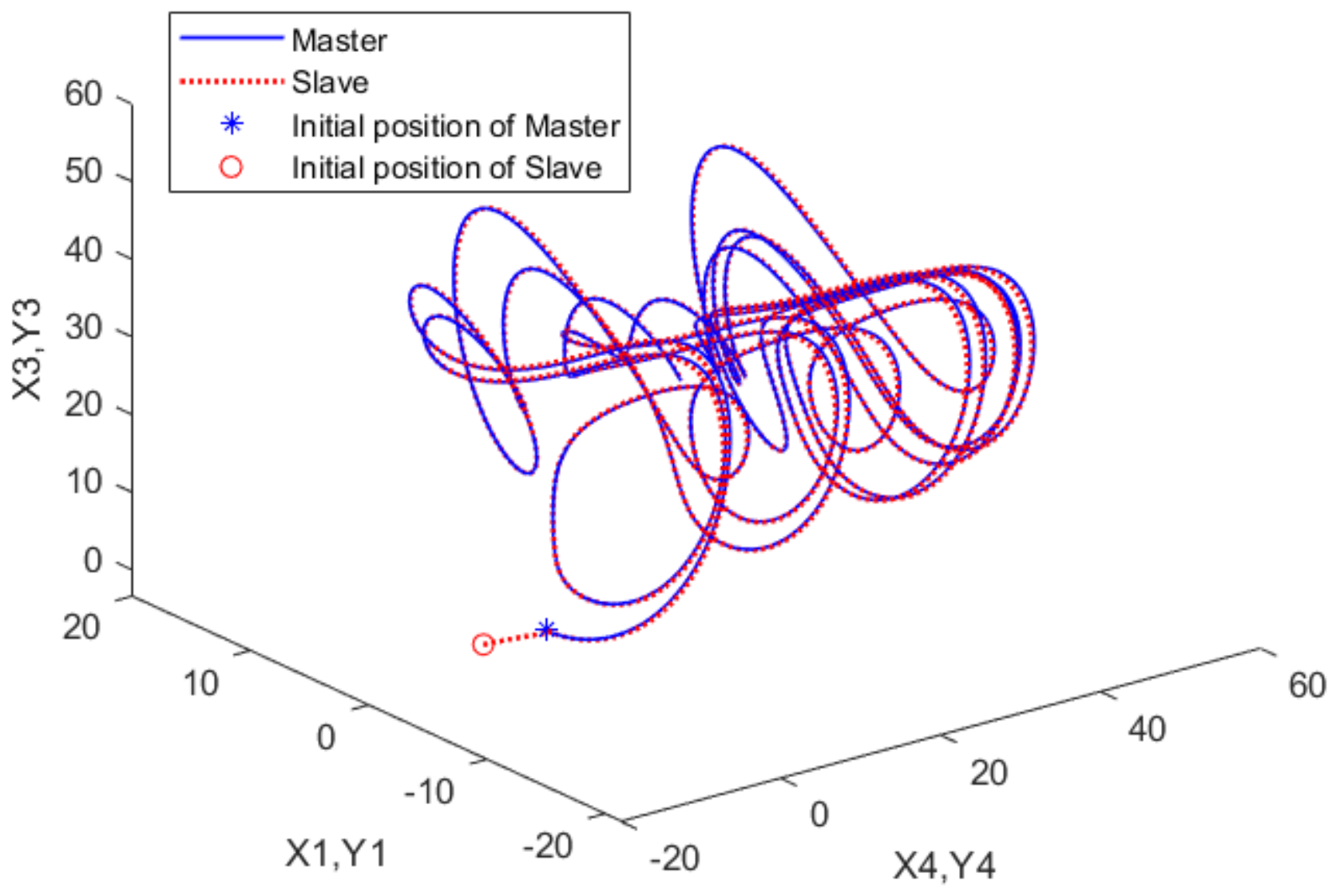

Figure 7

3D trajectory phase portrait of master $(X 1, X 3, X 4)$ and slave $(Y 1, Y 3, Y 4)$ 


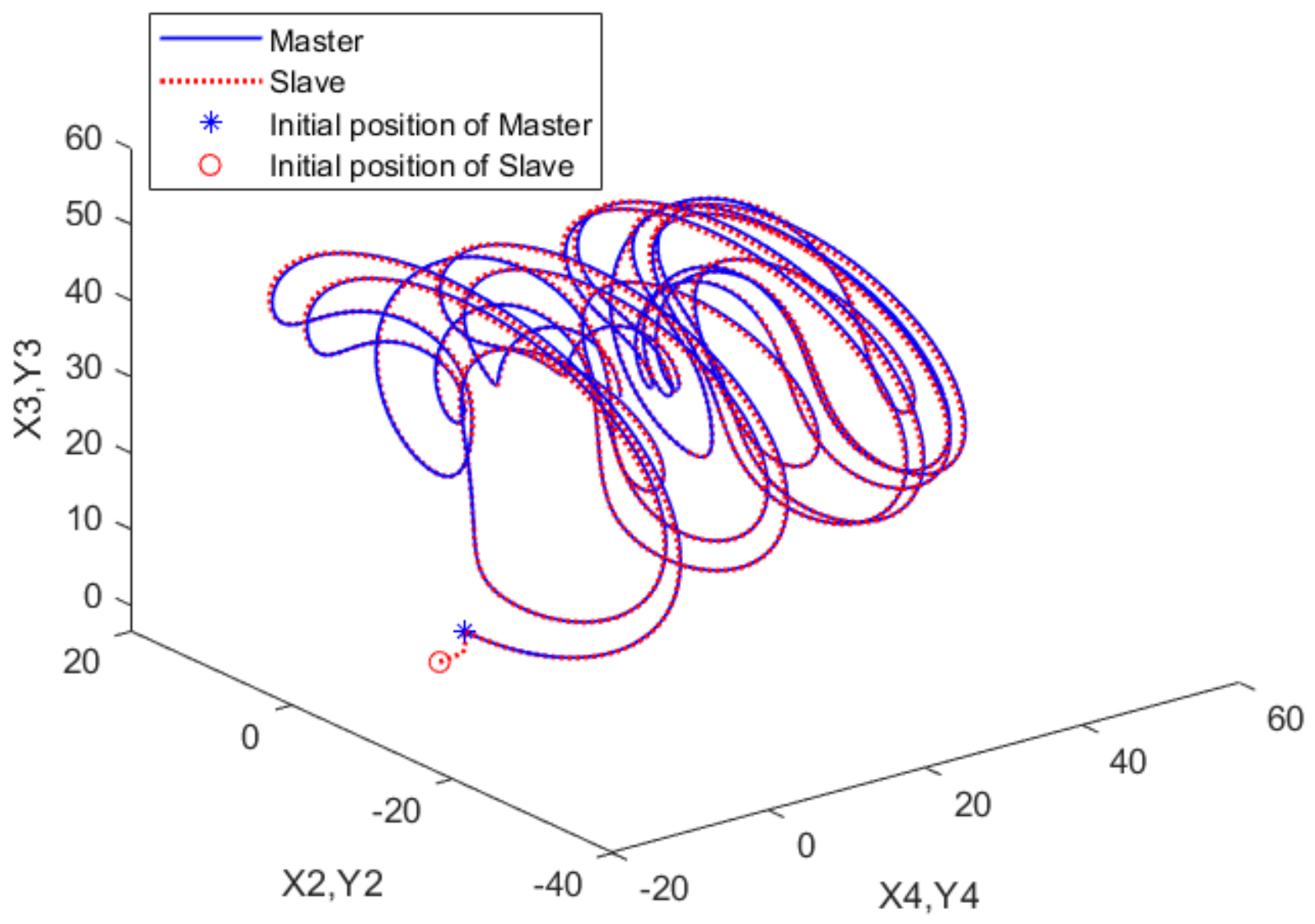

Figure 8

3D trajectory phase portrait of master $(X 2, X 3, X 4)$ and slave $(Y 2, Y 3, Y 4)$ 


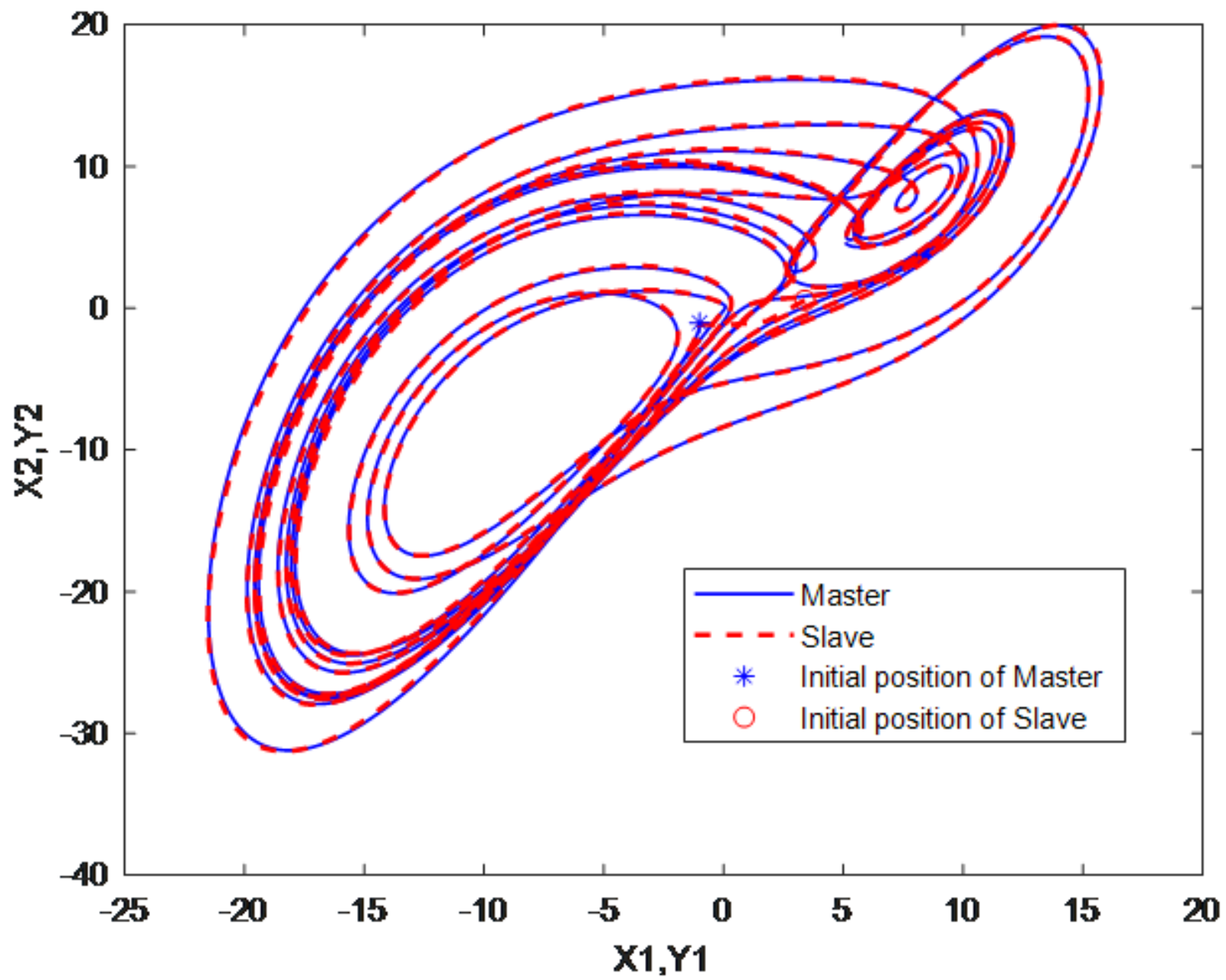

Figure 9

The state trajectory for master $(\mathrm{X} 1, \mathrm{X} 2)$ and slave $(\mathrm{Y} 1, \mathrm{Y} 2)$ 


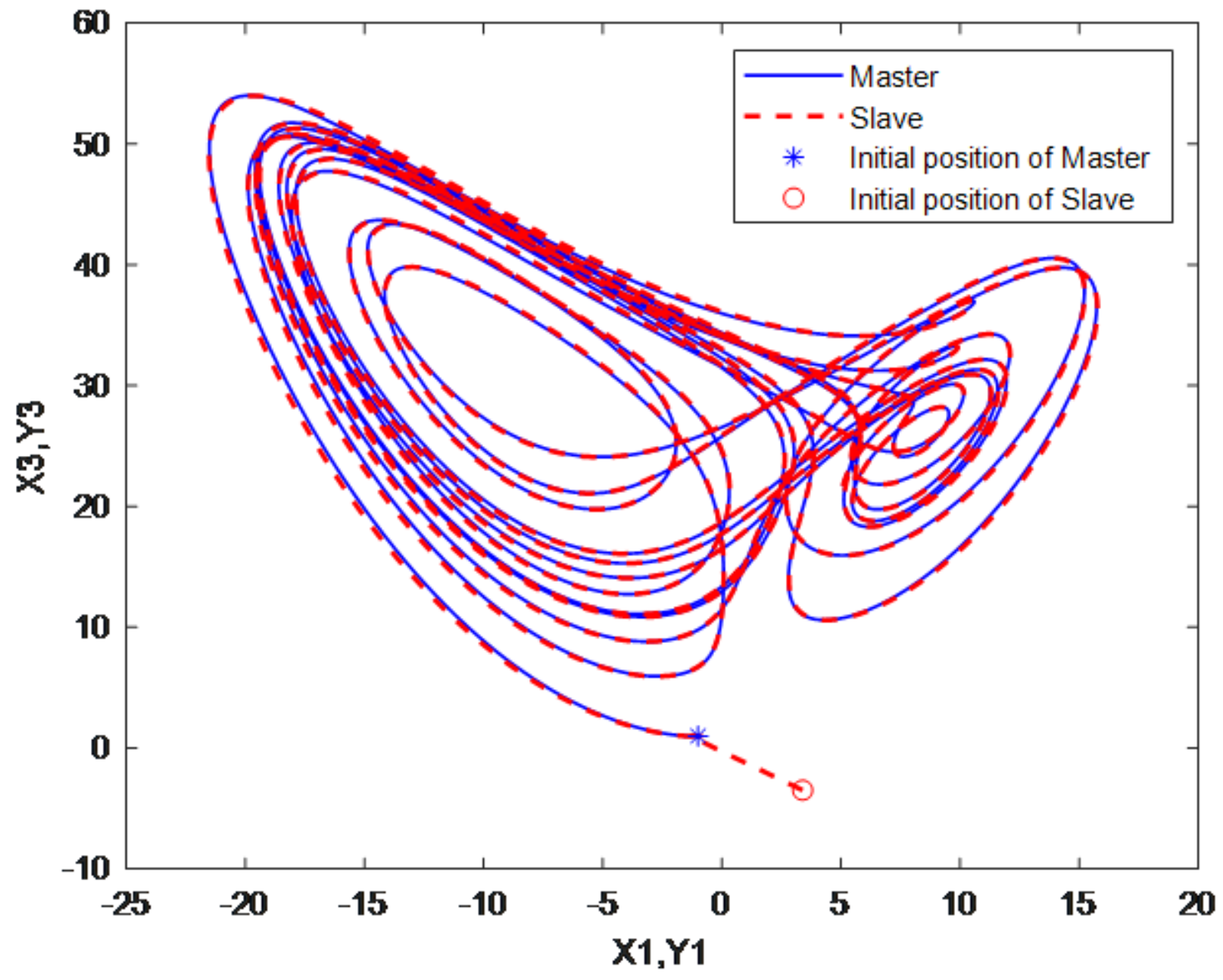

Figure 10

The state trajectory for master $(\mathrm{X} 1, \mathrm{X} 3)$ and slave $(\mathrm{Y} 1, \mathrm{Y} 3)$ 


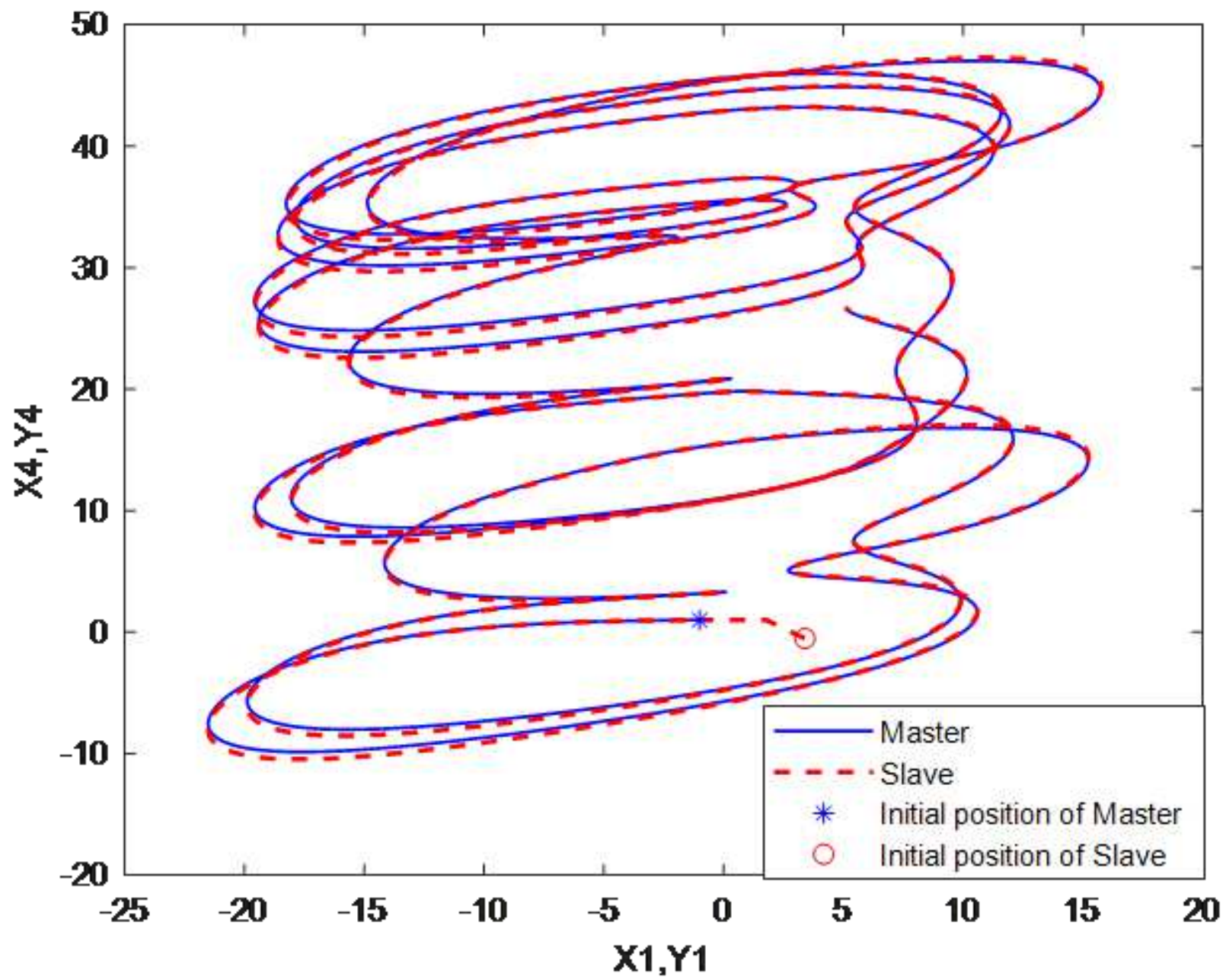

Figure 11

The state trajectory for master $(\mathrm{X} 1, \mathrm{X} 4)$ and slave $(\mathrm{Y} 1, \mathrm{Y} 4)$ 


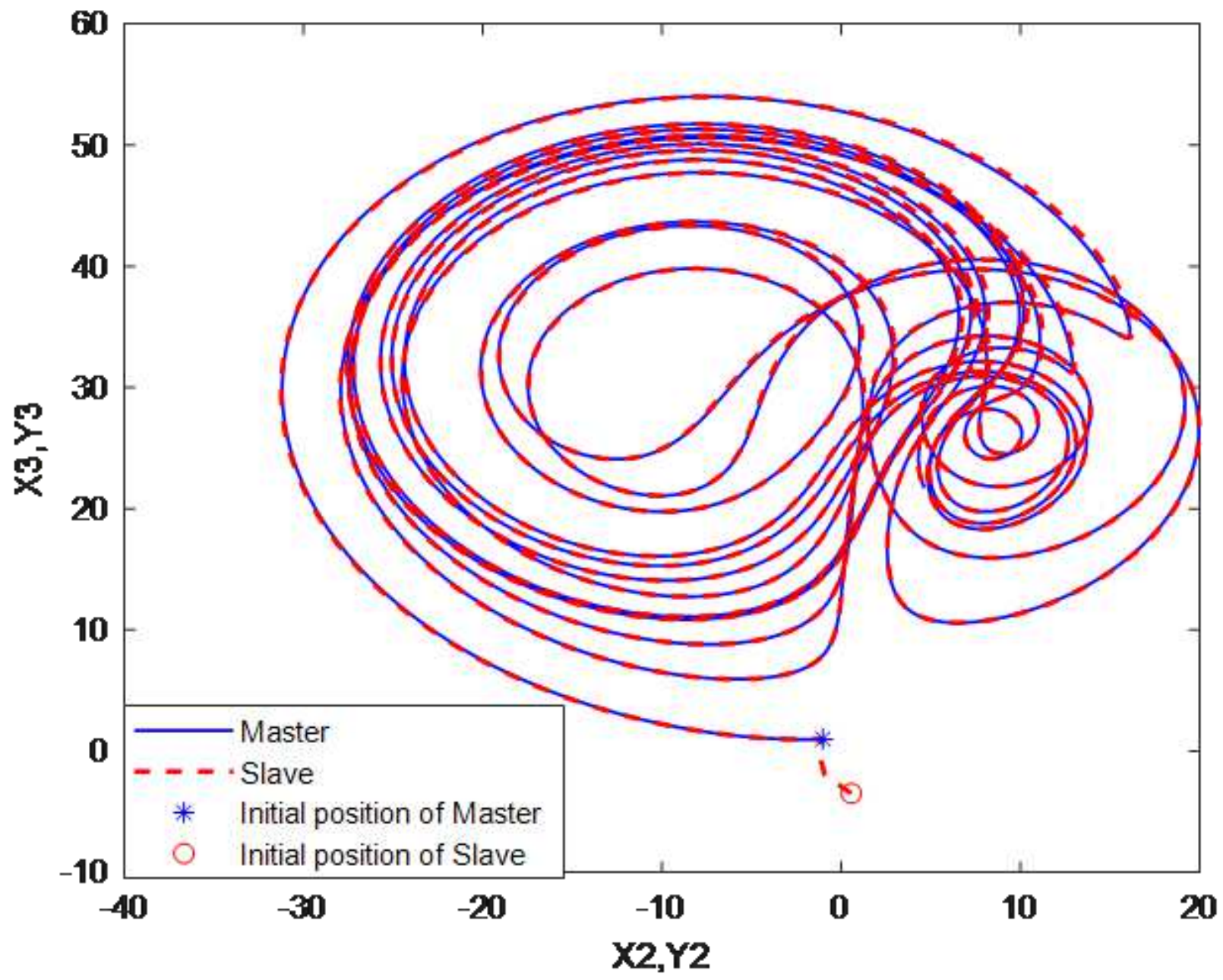

Figure 12

The state trajectory for master $(\mathrm{X} 2, \mathrm{X} 3)$ and slave $(\mathrm{Y} 2, \mathrm{Y} 3)$ 


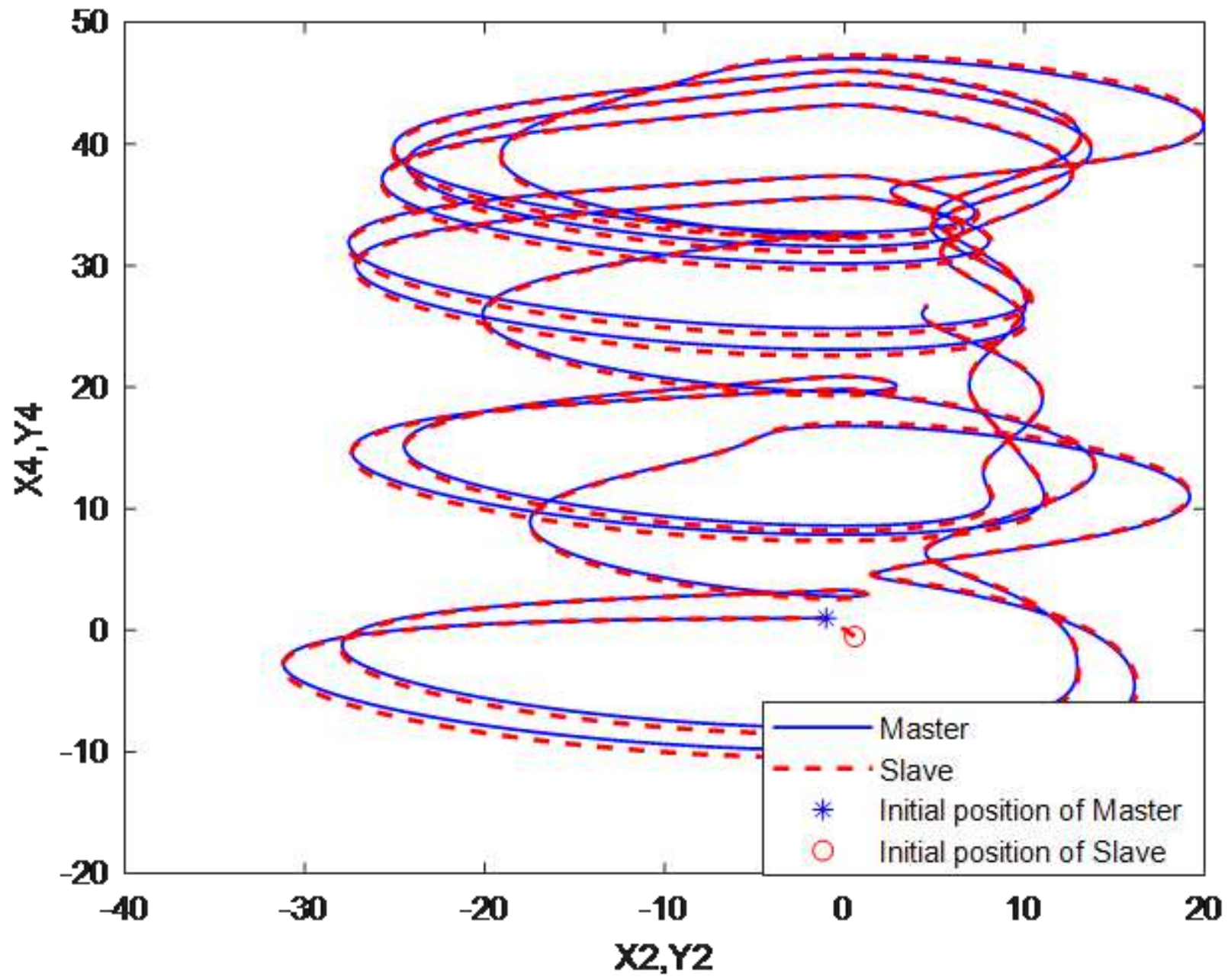

Figure 13

The state trajectory for master $(\mathrm{X} 2, \mathrm{X} 4)$ and slave $(\mathrm{Y} 2, \mathrm{Y} 4)$ 


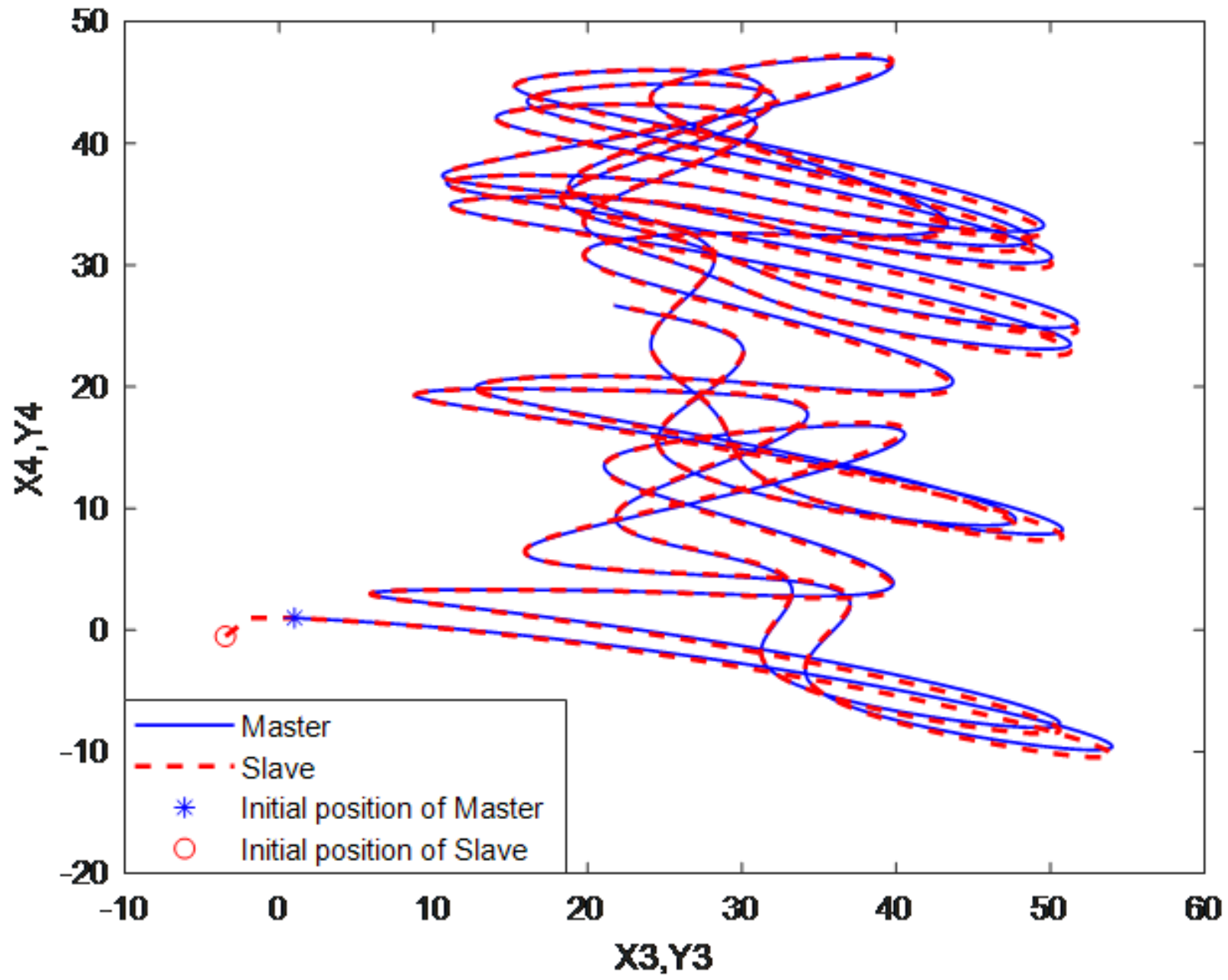

Figure 14

The state trajectory for master $(\mathrm{X} 3, \mathrm{X} 4)$ and slave $(\mathrm{Y} 3, \mathrm{Y} 4)$ 

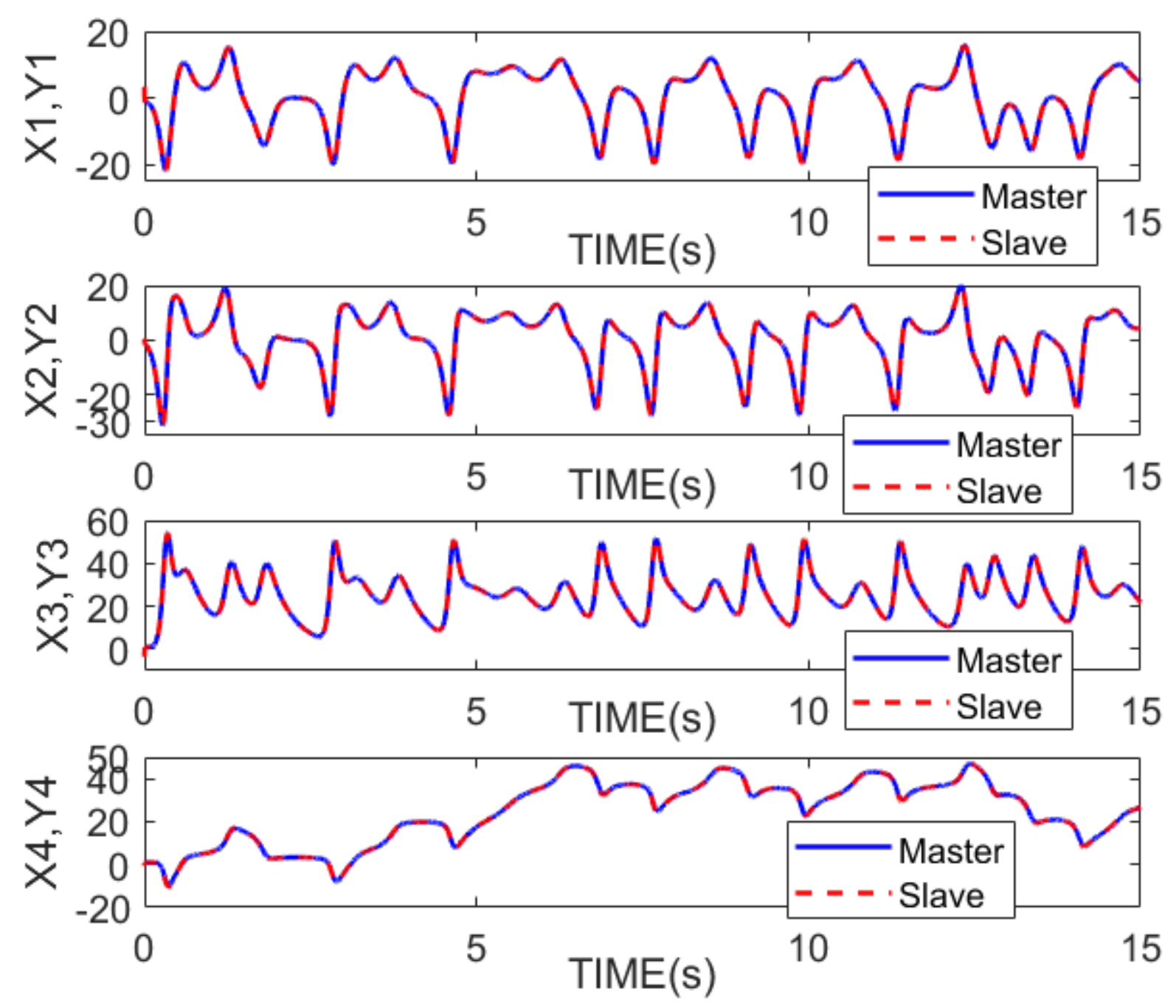

Figure 15

State trajectories for master-slave (X1, Y1), $(\mathrm{X} 2, \mathrm{Y} 2),(\mathrm{X} 3, \mathrm{Y} 3)$ and $(\mathrm{X} 4, \mathrm{Y} 4)$ 

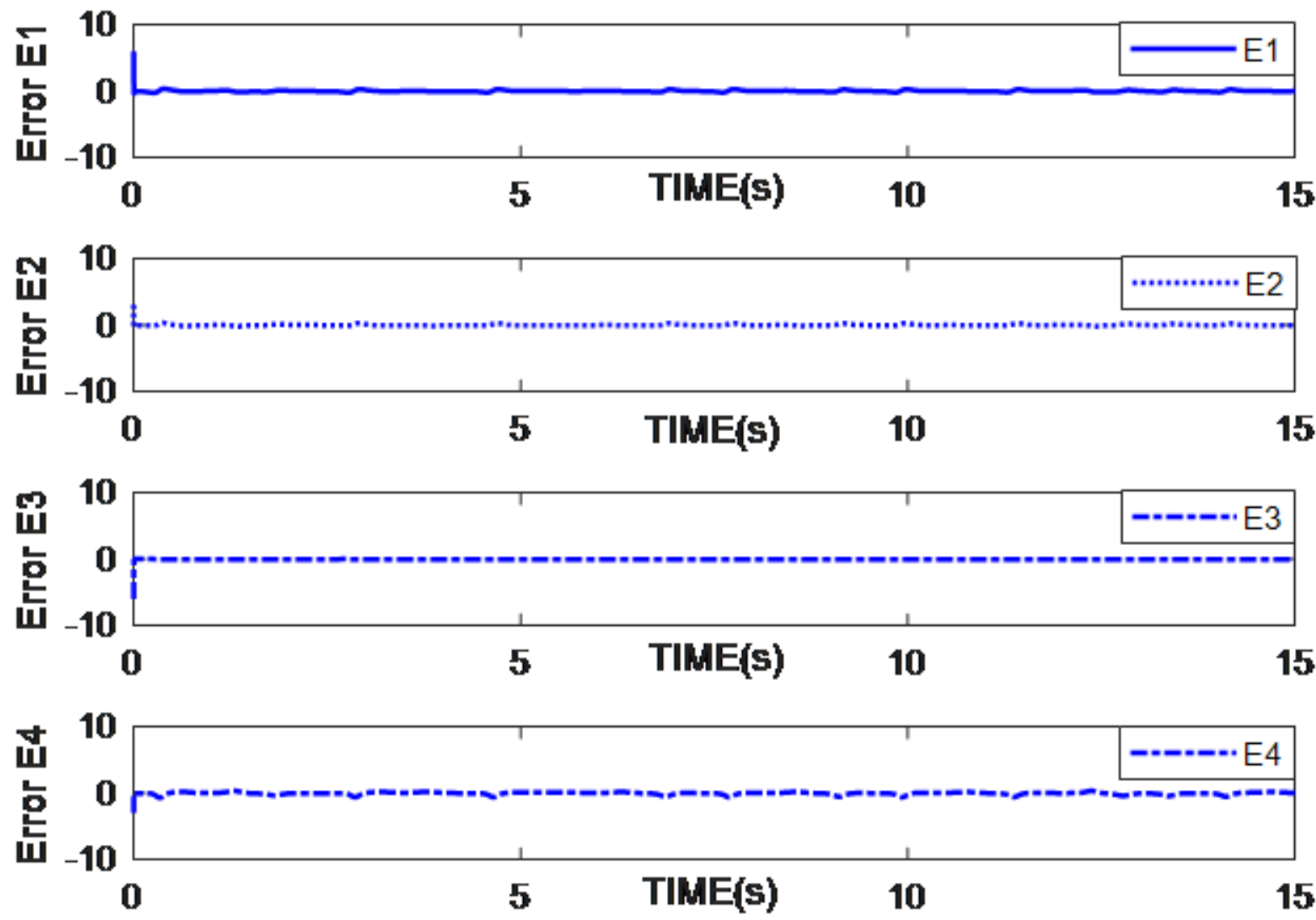

Figure 16

Errors for synchronization of the 4D hyperchaotic Lorenz-Lu system 


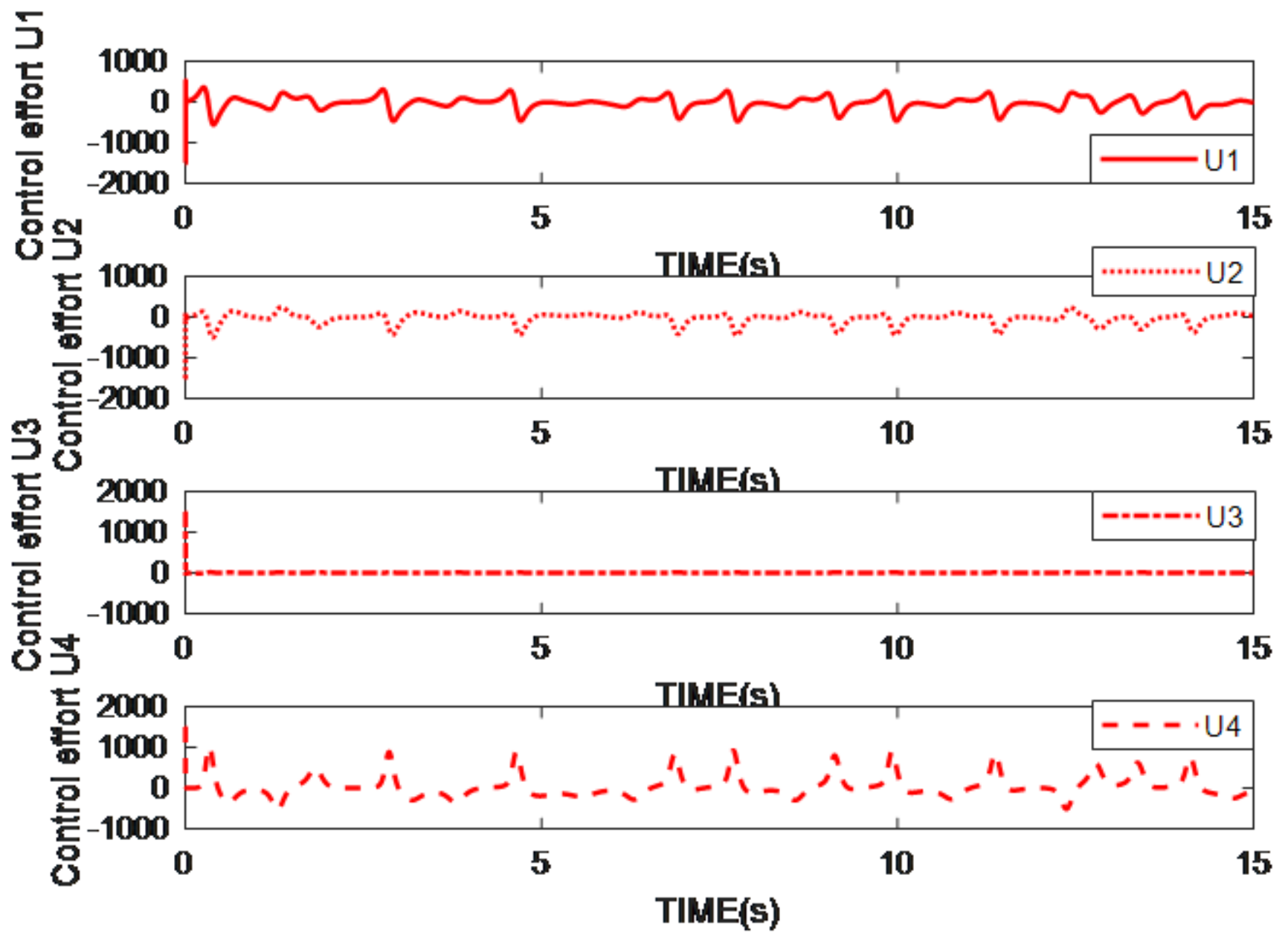

Figure 17

Control efforts for synchronization of the 4D hyperchaotic Lorenz-Lu system 

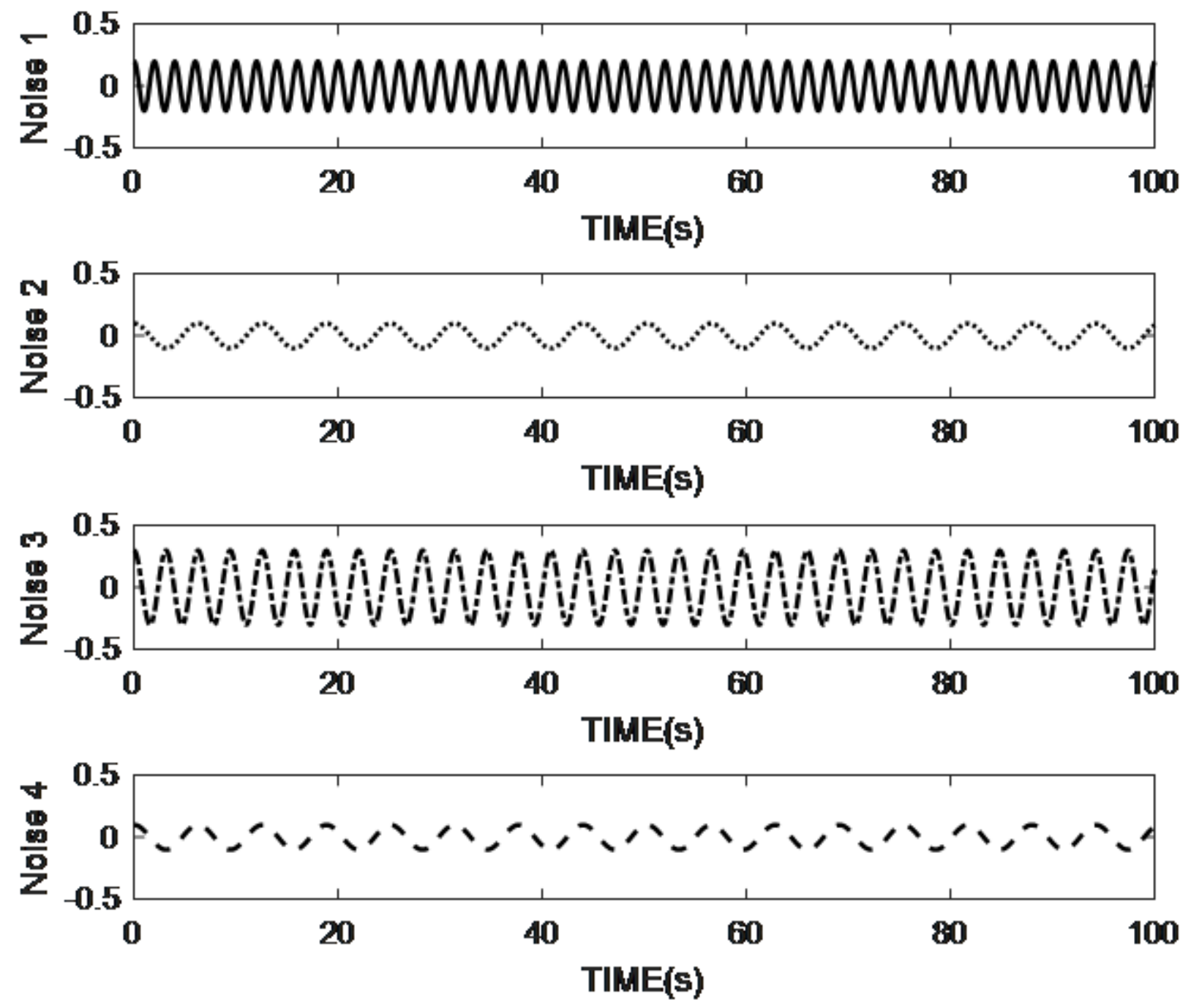

Figure 18

External noises using for synchronization of the 4D hyperchaotic Rikitake Dynamo System 


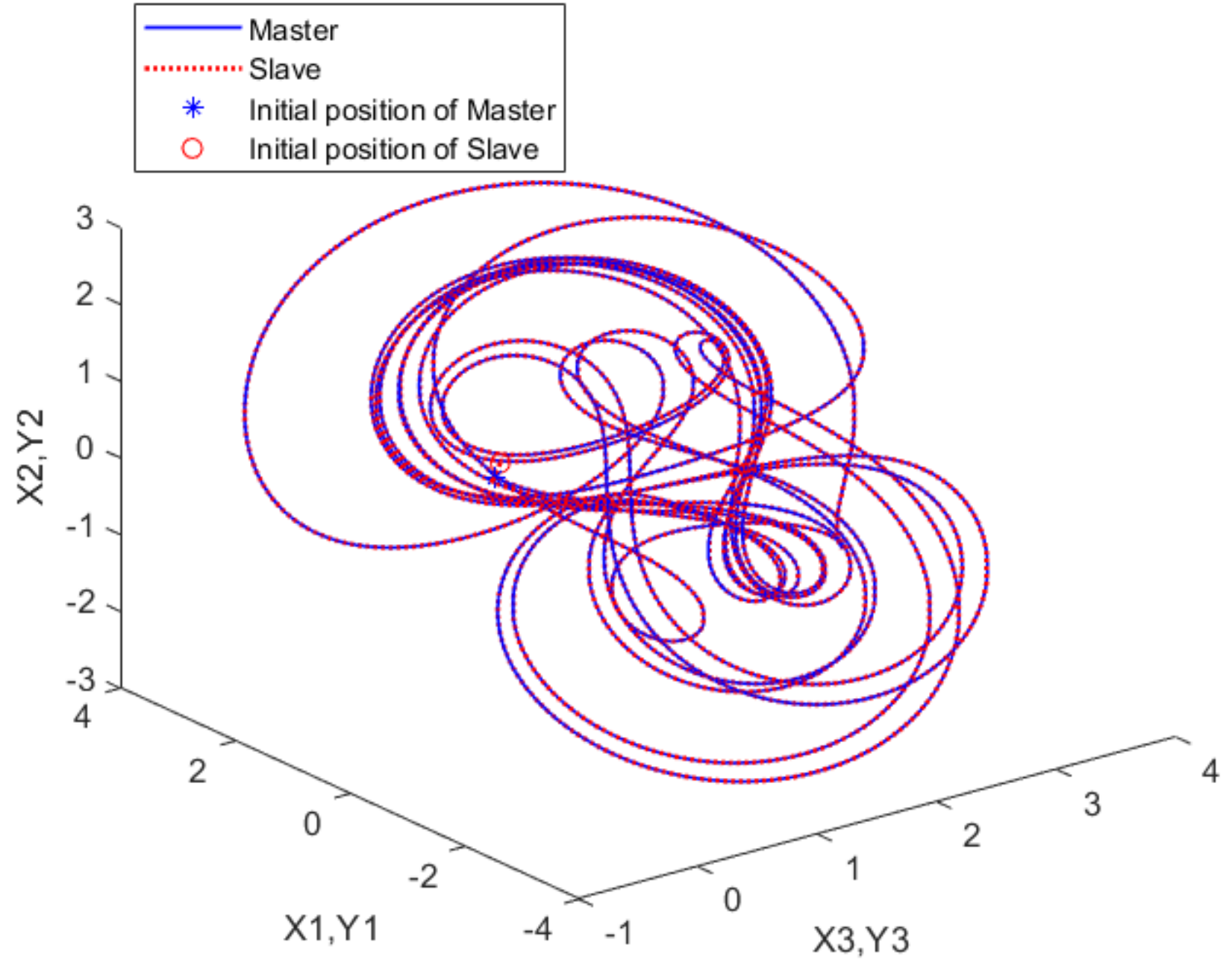

Figure 19

3D trajectory phase portrait of master $(\mathrm{X} 1, \mathrm{X} 2, \mathrm{X} 3)$ and slave $(\mathrm{Y} 1, \mathrm{Y} 2, \mathrm{Y} 3)$ 


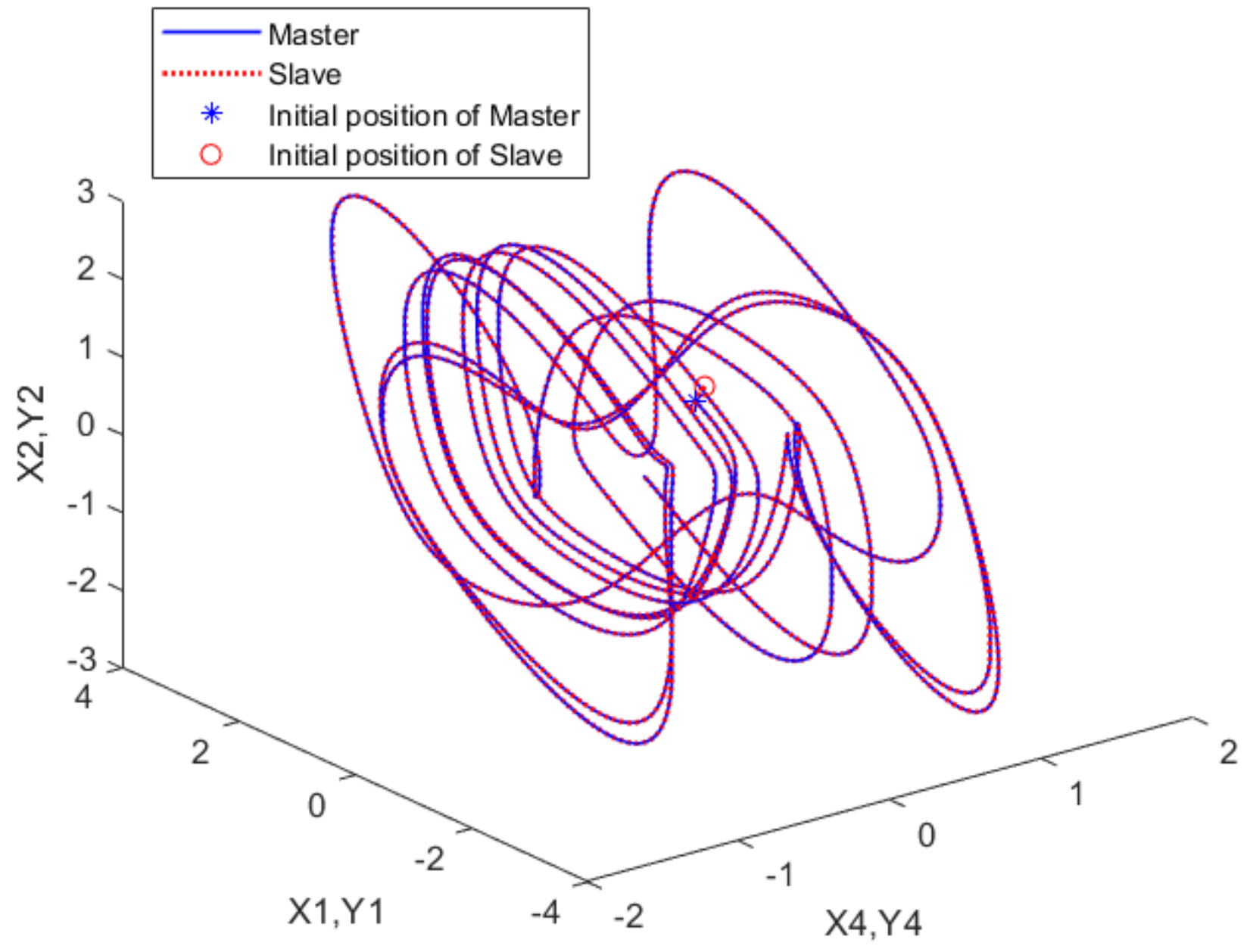

Figure 20

3D trajectory phase portrait of master $(\mathrm{X} 1, \mathrm{X} 2, \mathrm{X} 4)$ and slave $(\mathrm{Y} 1, \mathrm{Y} 2, \mathrm{Y} 4)$ 


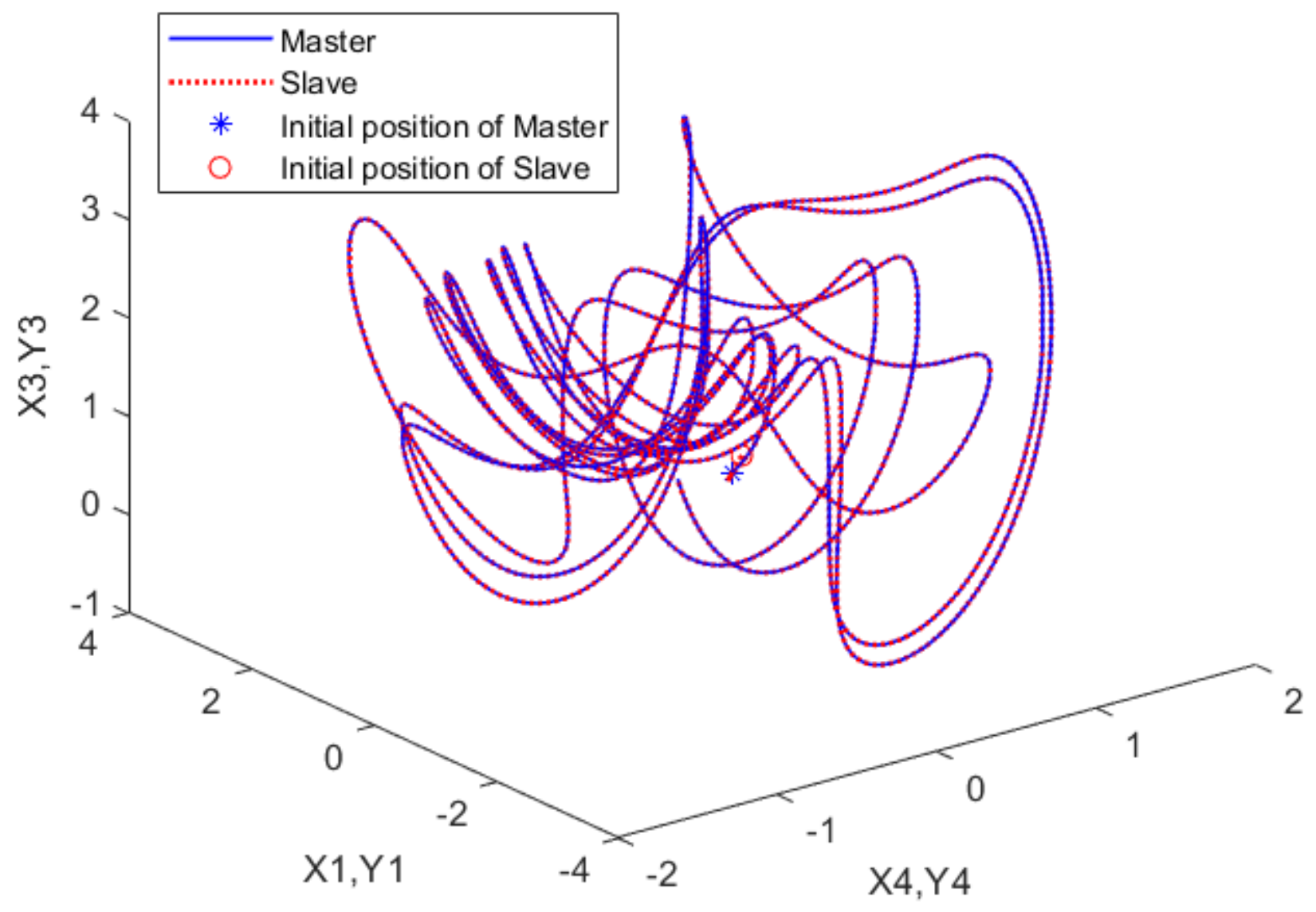

Figure 21

$3 \mathrm{D}$ trajectory phase portrait of master $(\mathrm{X} 1, \mathrm{X} 3, \mathrm{X} 4)$ and slave $(\mathrm{Y} 1, \mathrm{Y} 3, \mathrm{Y} 4)$ 


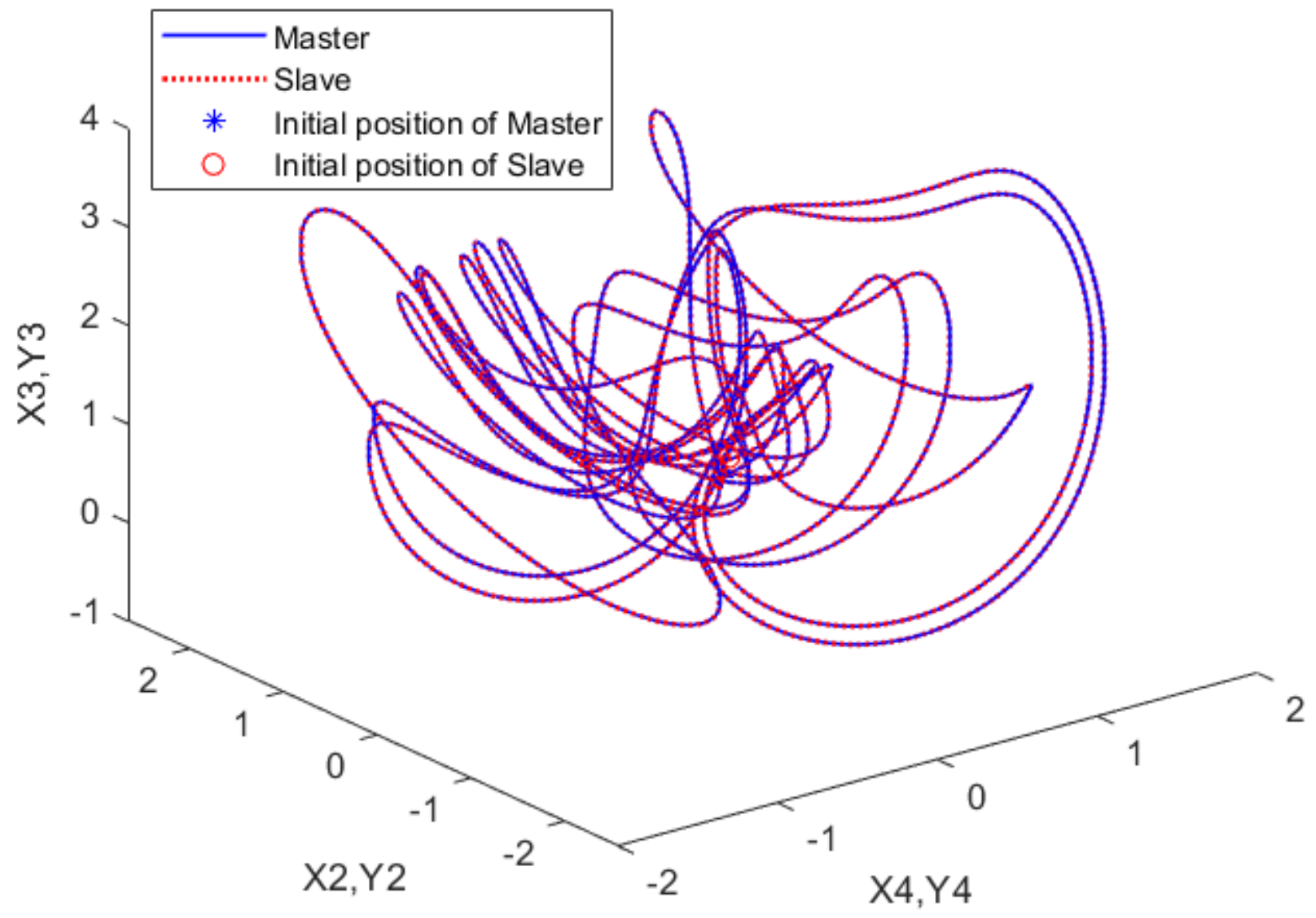

Figure 22

3D trajectory phase portrait of master $(X 2, X 3, X 4)$ and slave $(Y 2, Y 3, Y 4)$ 


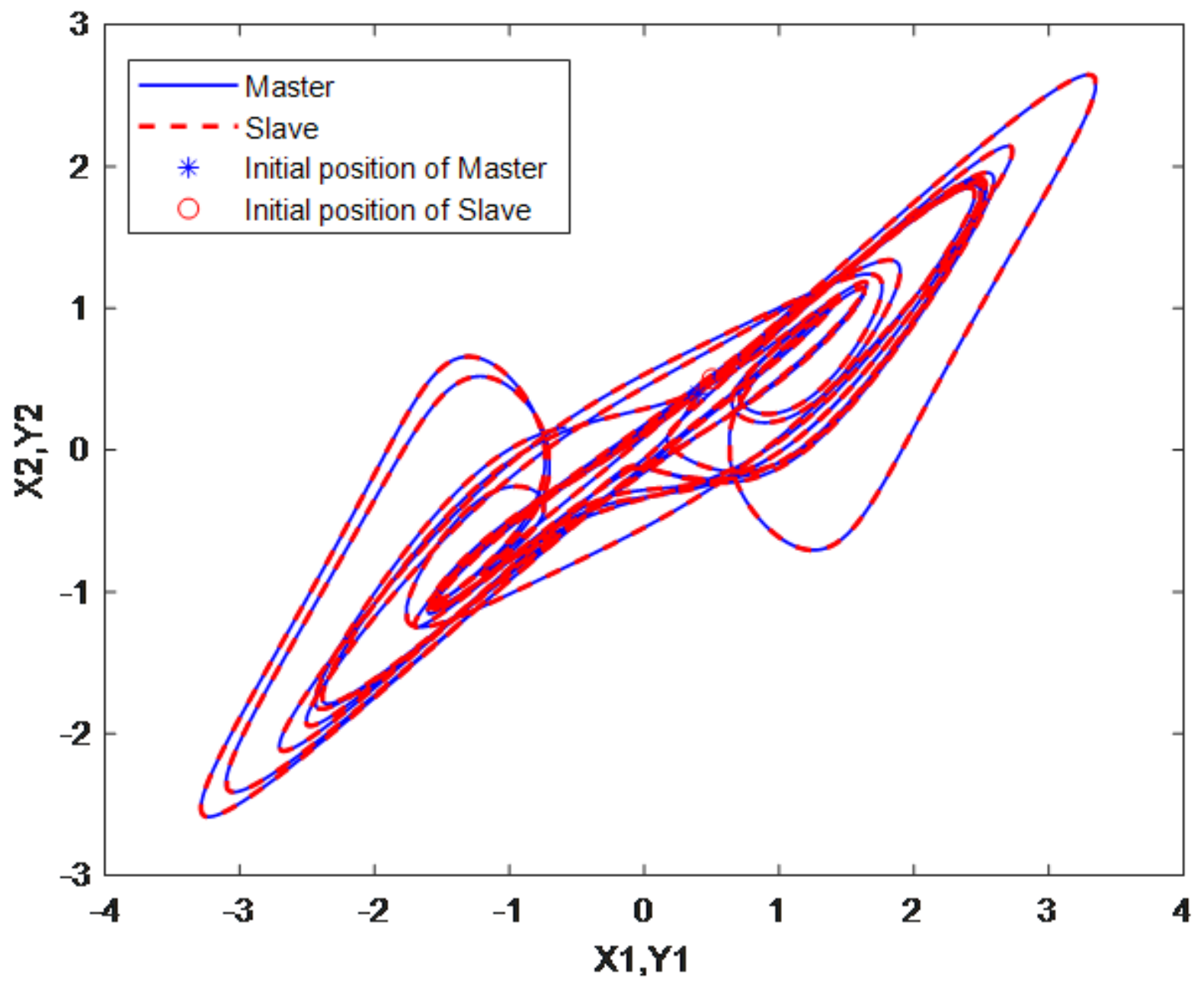

Figure 23

The state trajectory of master $(\mathrm{X} 1, \mathrm{X} 2)$ and slave $(\mathrm{Y} 1, \mathrm{Y} 2)$ 


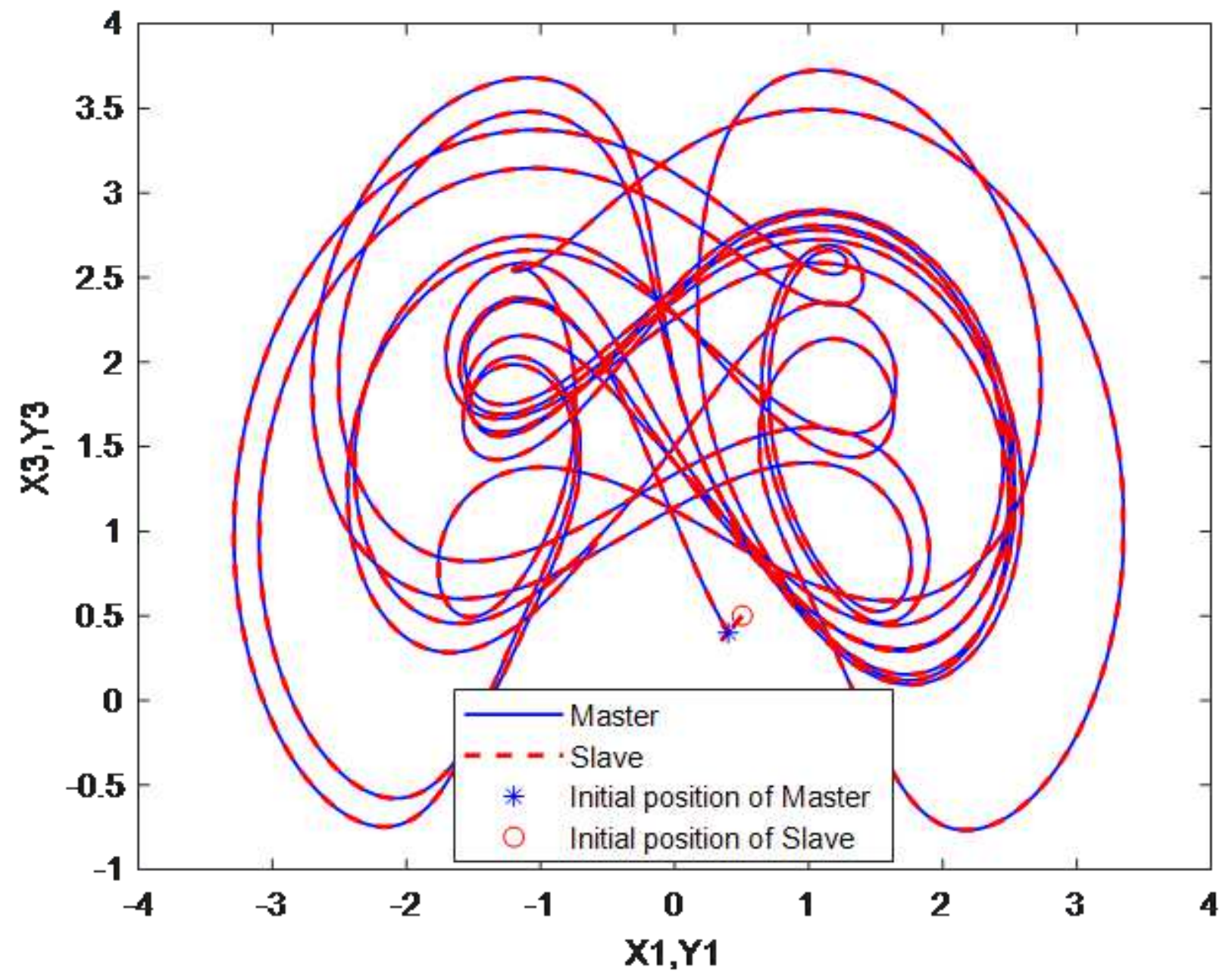

Figure 24

The state trajectory of master $(\mathrm{X} 1, \mathrm{X} 3)$ and slave $(\mathrm{Y} 1, \mathrm{Y} 3)$ 


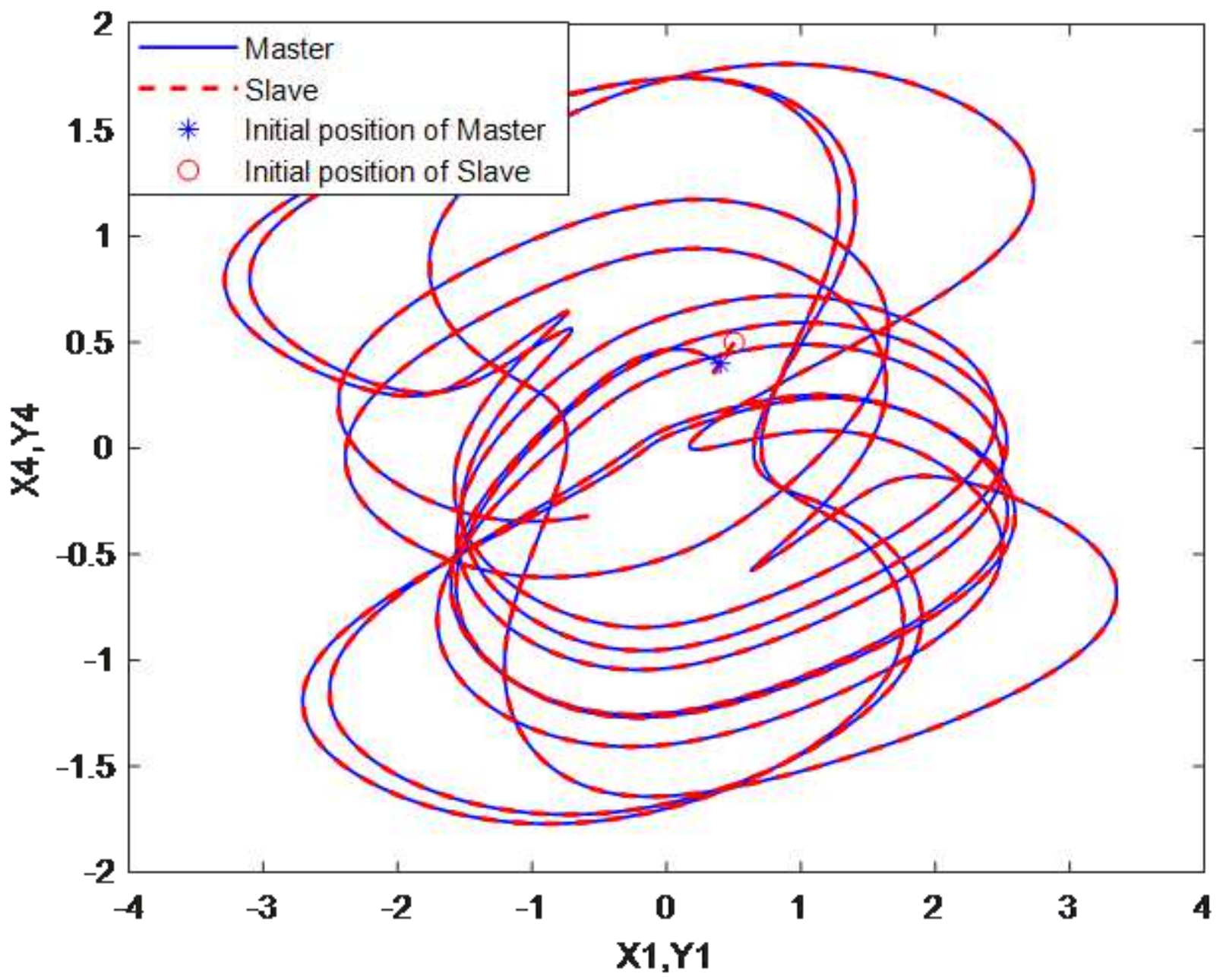

Figure 25

The state trajectory of master $(\mathrm{X} 1, \mathrm{X} 4)$ and slave $(\mathrm{Y} 1, \mathrm{Y} 4)$ 


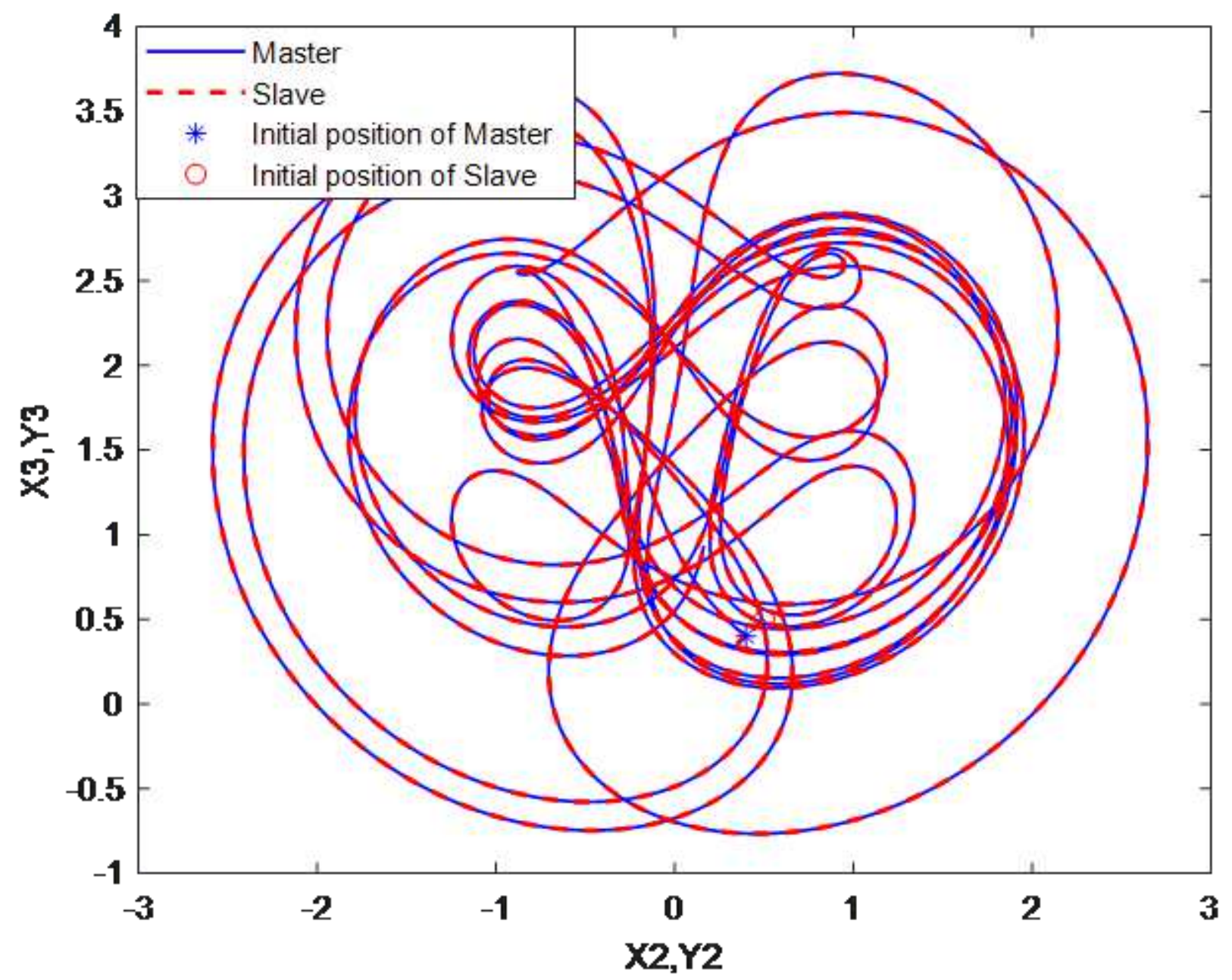

Figure 26

The state trajectory of master $(\mathrm{X} 2, \mathrm{X} 3)$ and slave $(\mathrm{Y} 2, \mathrm{Y} 3)$ 


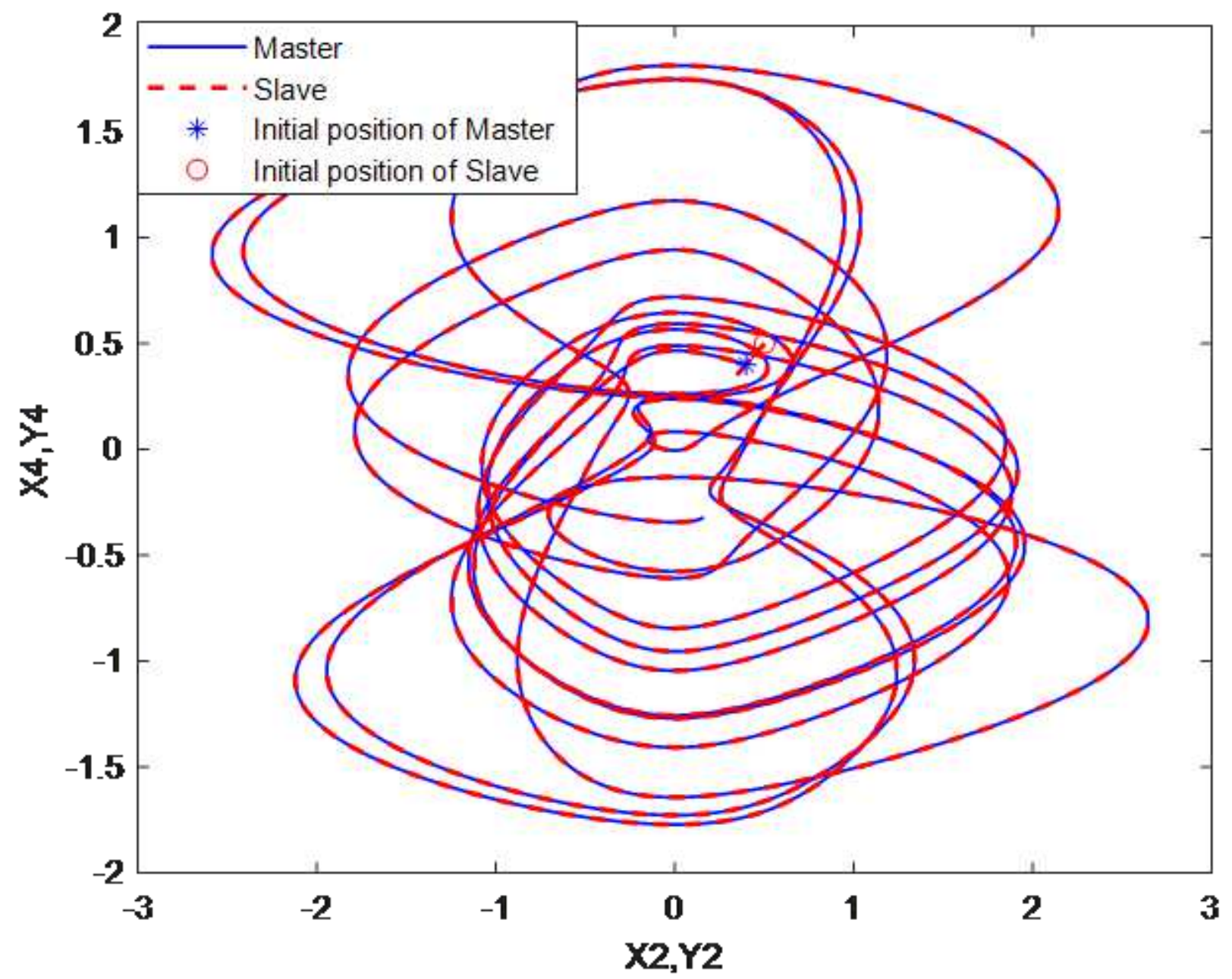

Figure 27

The state trajectory of master $(\mathrm{X} 2, \mathrm{X} 4)$ and slave $(\mathrm{Y} 2, \mathrm{Y} 4)$ 


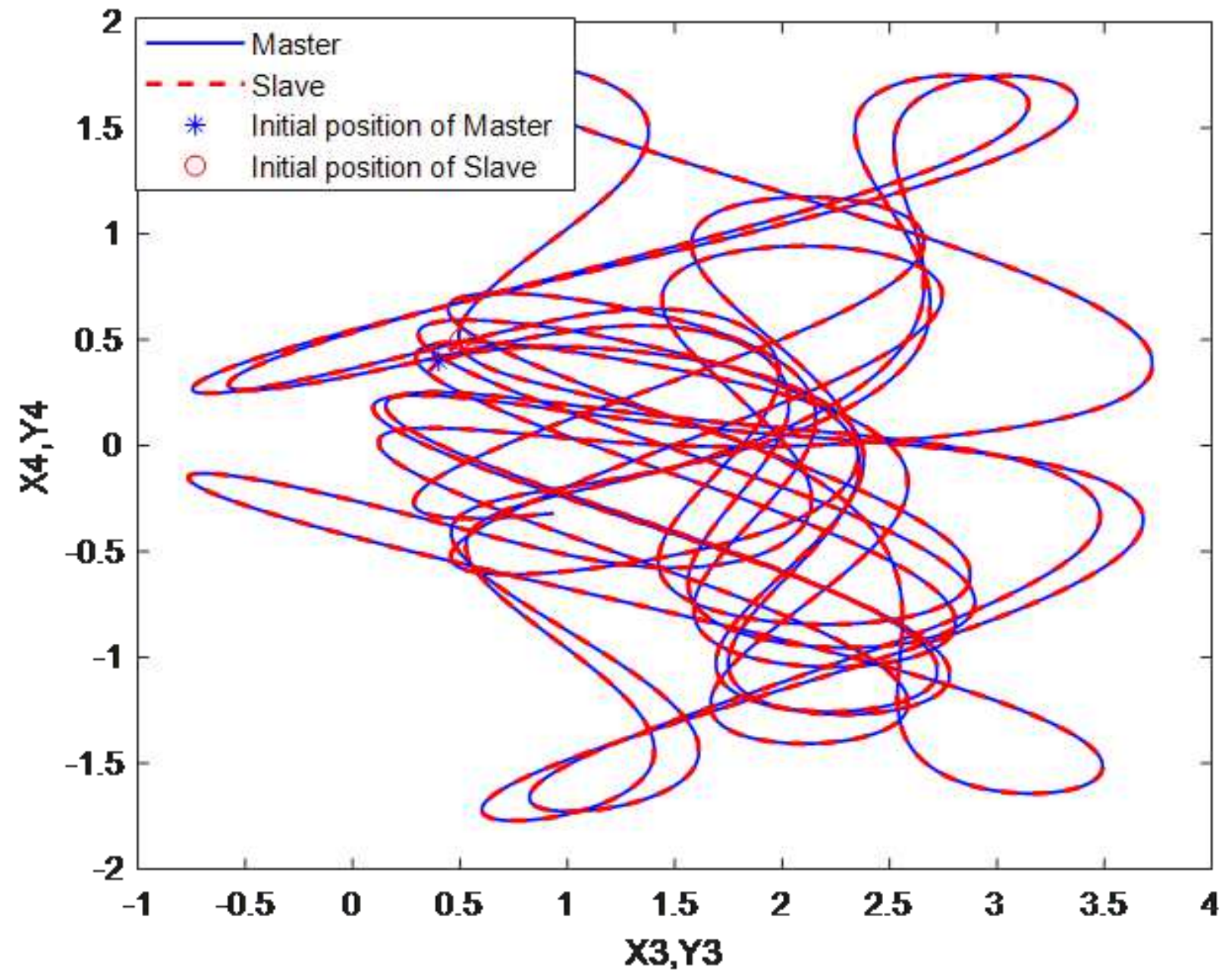

Figure 28

The state trajectory of master $(\mathrm{X} 3, \mathrm{X} 4)$ and slave $(\mathrm{Y} 3, \mathrm{Y} 4)$ 

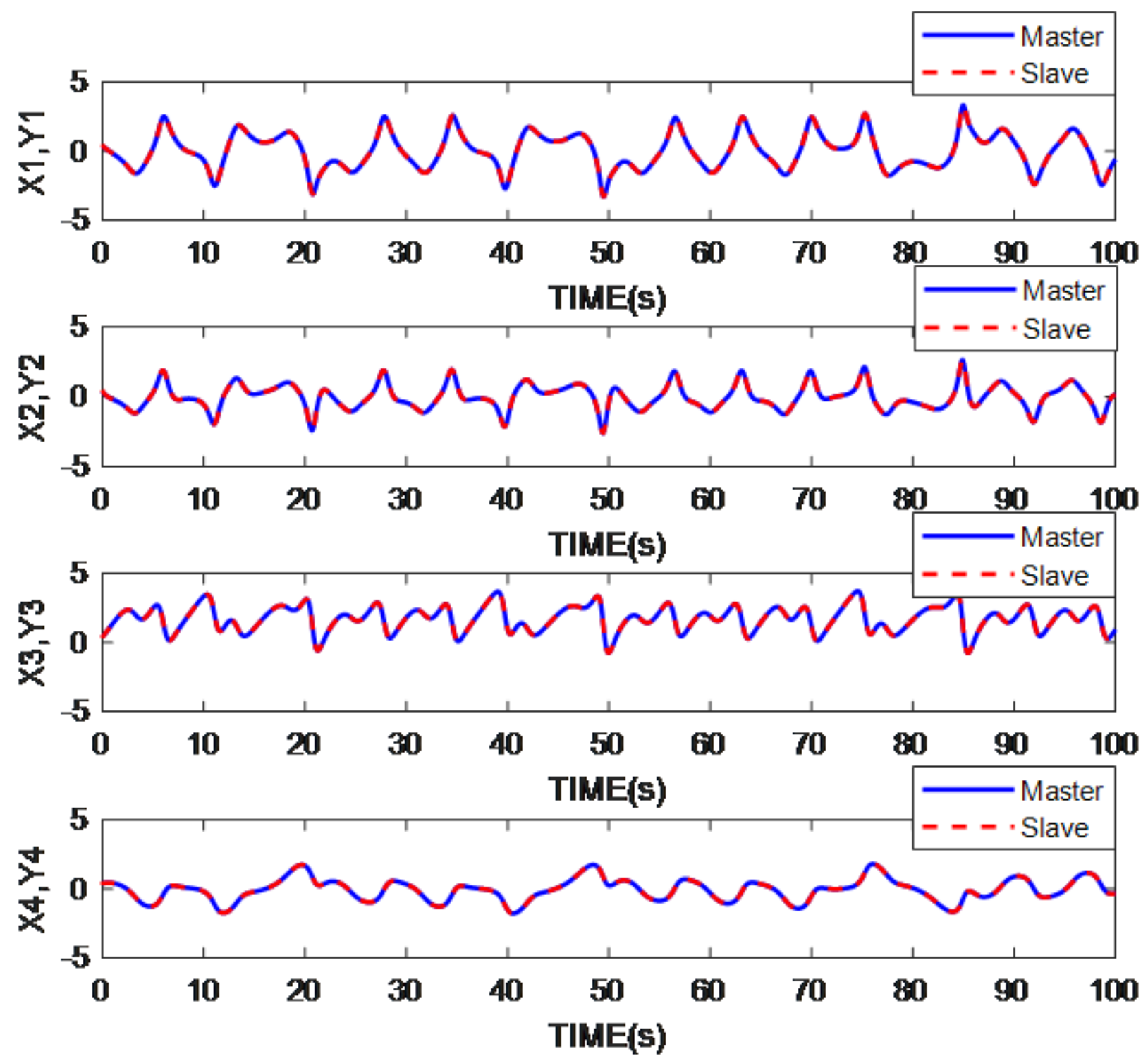

Figure 29

The state trajectory of master-slave (X1, Y1), (X2, Y2), (X3, Y3), and (X4, Y4) 

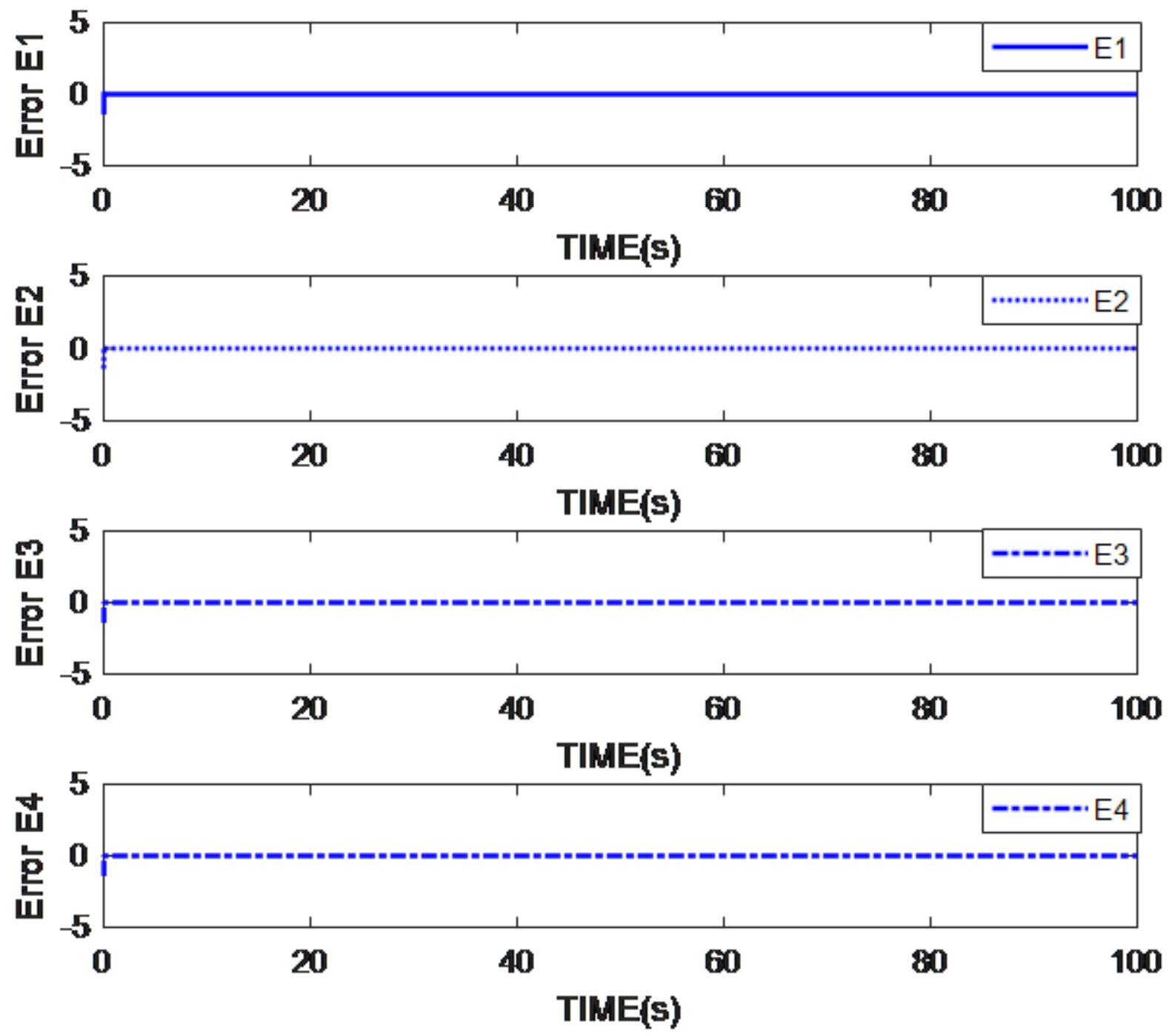

Figure 30

The errors of synchronization for the 4D hyperchaotic Rikitake Dynamo System 


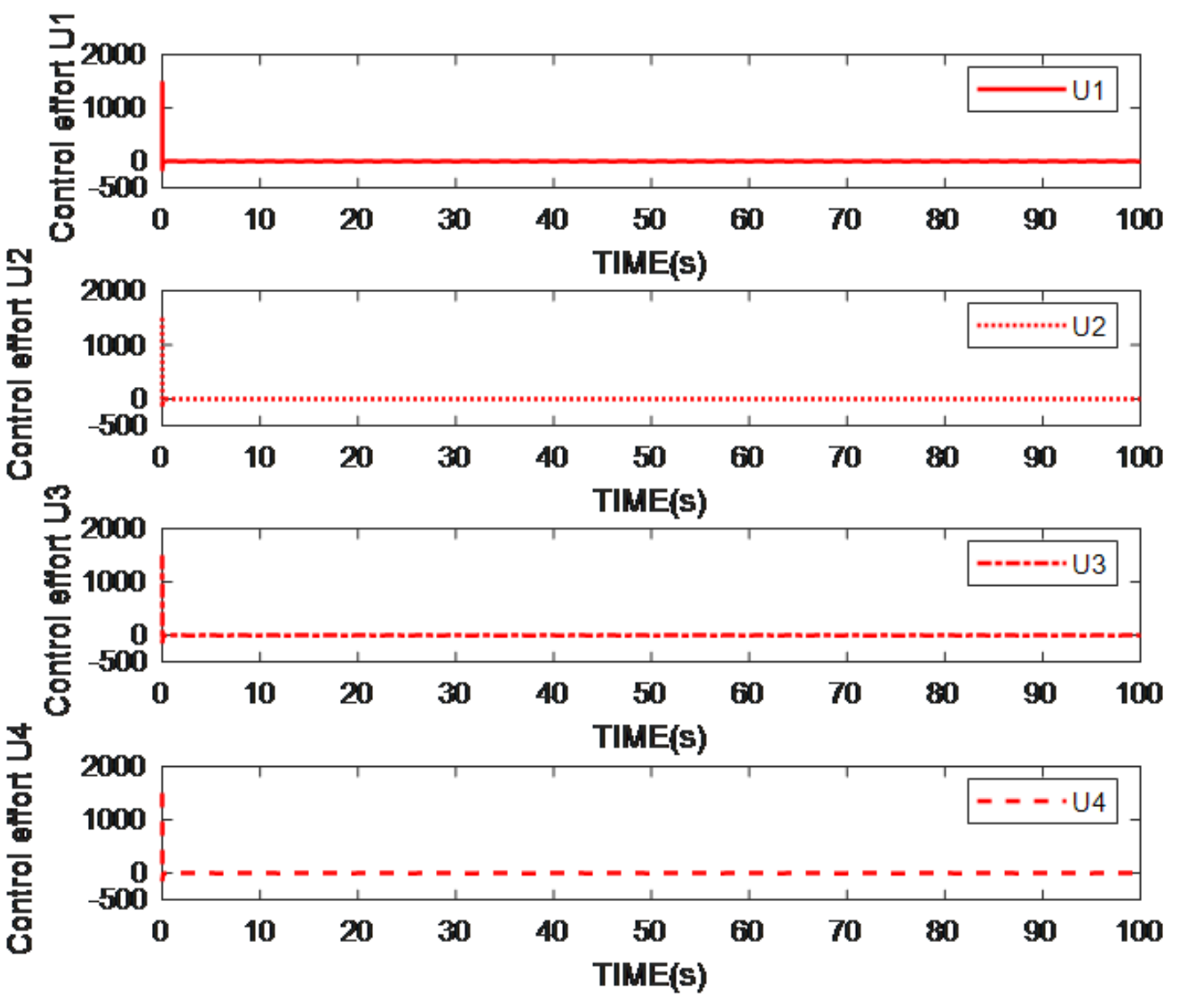

Figure 31

The control efforts of synchronization for the 4D hyperchaotic Rikitake Dynamo System 